\title{
Stochastic Geometry-Based Tools for Spatial Modeling and Planning of Future Cellular Networks: Opportunistic Cell Switch-off and Strategic Deployment of Flying Base Stations
}

\author{
by \\ Faraj Lagum, B. Sc., M. Sc. \\ Doctor of Philosophy in Electrical and Computer Engineering \\ Ottawa-Carleton Institute for Electrical and Computer Engineering \\ Department of Systems and Computer Engineering \\ Carleton University \\ Ottawa, Ontario \\ November 2018 \\ (C) Copyright \\ Faraj Lagum, 2018
}


The undersigned hereby recommends to the

Faculty of Graduate and Postdoctoral Affairs acceptance of the dissertation

\title{
Stochastic Geometry-Based Tools for Spatial Modeling and Planning of Future Cellular Networks: Opportunistic Cell Switch-off and Strategic Deployment of Flying Base Stations
}

\author{
submitted by Faraj Lagum, B. Sc., M. Sc. \\ in partial fulfillment of the requirements for the degree of \\ Doctor of Philosophy in Electrical and Computer Engineering \\ Professor Halim Yanikomeroglu, Thesis Supervisor \\ Dr. Sebastian S. Szyszkowicz, Thesis Co-supervisor \\ Ottawa-Carleton Institute for Electrical and Computer Engineering \\ Department of Systems and Computer Engineering \\ Carleton University \\ November 2018
}




\section{Abstract}

To meet the ever-increasing traffic demand in modern cellular networks, deploying more BSs has imposed is a vital solution. Cellular network (re-)planning is crucial to mitigate the interference and improve the network performance whenever more base stations (BSs) are deployed or some BSs are removed. Realistic spatial modelling for the locations of BSs and accurate quantification for their spatial relationship are prerequisites for effective network planning.

In the first part of this thesis, we aim to describe the spatial structure of the BSs using two scalar measures: the density of the BSs and the amount of regularity. We investigate the use of three scalar metrics to measure the spatial relationships among the BSs in cellular networks. We propose a geometry-based scalar metric (the coefficient of variation $(\mathrm{CoV})$ of the length of the corresponding edges of Delaunay triangulation) to quantify the spatial regularity of the repulsive wireless networks. This work develops new approaches for i) mapping between the internal parameters of different point processes commonly used to generate the BS locations, ii) approximating the performance of a repulsive network based on its amount of regularity, and iii) fitting point processes to the spatial deployment of BSs.

In the second part, we develop a novel stochastic geometry-based cellular network planning technique that relies on the spatial structure of the network to determine the best deployment or removal locations of the BSs. First, we apply this technique for cell deactivation during the low demand periods. More specifically, cells are switched off so that the remaining active cells are as far away as possible from each other, which maximizes the spatial regularity of the network. The results show significant energy saving and network performance enhancement. Second, we exploit this approach for the strategic densification with UAV-BSs in cellular networks: The deployment of multiple UAV-BSs in the presence of a terrestrial network where the UAV-BSs provide on-demand capacity to the end users. This study provides supply-side estimation for how many UAV-BSs are needed to support a terrestrial network so as to achieve a 
particular quality-of-service and also demonstrate where these UAV-BSs should hover. 
Dedicated to whom this thesis matters most,

My parents, Suliman and Saddina, my wife, Nisreen, and my childeren, Suliman and Rose. 


\section{Acknowledgments}

In the name of Allah, the Most Gracious, the Most Merciful. All the praise is due purely to Allah, alone, for His countless blessings and bounties.

I would like to express my sincere gratitude to my supervisor, Prof. Halim Yanikomeroglu, for his amazing leadership skills, flexibility, and encouragement. His

advice and guidance have pushed me far beyond my expectations. It is my privilege to have an opportunity to work with him. My sincere thanks must also go to my co-supervisor, Dr. Sebastian S. Szyszkowicz. He taught me how to sharpen my skills and set up exacting standards for my research.

I would like to thank the members of the examining committee for their valuable discussion and for their unanimous decision to nominate me to a Carleton University medal.

I would express my thanks to Dr. Rainer Schoenen, Hamburg University of Applied Sciences, Germany, for advising me to take this elegant research direction, and for his comments and feedback on my early work. I should also thank my senior colleague and friend, Meisam Mirahsan, for explaining his work to me, which I built my research on. I am also thankful to my colleagues in our research group for making this long journey joyful.

To my parents, I am greatly indebted to their encouragement, emotional support, and prayers. To my wife, Nisreen, I am very thankful for your patience, generous support, and love. Special gratitude goes to my children, Suliman and Rose, for being the sweetest flavour in my life.

Finally, I thankfully acknowledge that my Ph.D. study is fully sponsored by the Libyan Ministry of Higher Education and Scientific Research through the LibyanNorth American Scholarship Program. 


\section{Table of Contents}

Abstract $\quad$ iii

Acknowledgments $\quad$ vi

Table of Contents vii

List of Tables $\quad$ xii

List of Figures xiii

Nomenclature $\quad$ xvii

List of Symbols $\quad$ xviii

1 Introduction 1

1.1 Motivation . . . . . . . . . . . . . . . . . . . 1

1.2 Literature Review . . . . . . . . . . . . . . . . . . . . 4

1.2.1 Point Process Models for Base Station Locations . . . . . . . . 4

1.2.2 Fitting Methods and Tools . . . . . . . . . . . . . . . 9

1.3 Scope and Objectives . . . . . . . . . . . . . . . . 10

1.4 Contributions ......................... 11

1.4.1 Proposing Scalar Metrics to Measure the Regularity (Irregularity) 11

1.4.2 Advocating Perturbed Triangular Lattice (PTL) Model . . . . 12

1.4.3 Creating a Novel Framework for Mapping between Point Processes . . . . . . . . . . . . . . . . . . . . . . 12

1.4.4 Proposing a New Approach for Approximating the Performance of the Repulsive Cellular Networks . . . . . . . . . . . . . . . 13

1.4.5 Proposing a New Approach for Fitting Point Processes to BSs 13 
1.4.6 Devising a Novel Energy Saving Approach . . . . . . . . . . 13

1.4.7 Devising Novel Strategies for Large-Scale UAV-BSs Placement 14

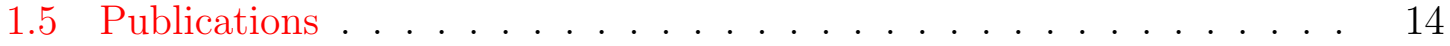

1.5.1 Journal Papers —Included in the Thesis . . . . . . . . . . . 14

1.5.2 Conference Paper —Included in the Thesis . . . . . . . . . 15

1.5.3 Journal Papers — Not Included in the Thesis . . . . . . . . 15

1.6 Organization of the Thesis . . . . . . . . . . . . . . . . 16

2 Geometry-Based Scalar Metrics to Quantify the Spatial Relationship among the Locations of the Base Stations 18

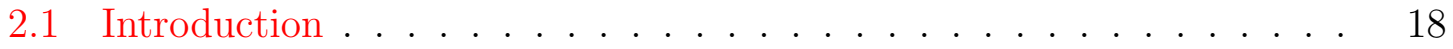

2.2 The Coefficient of Variation . . . . . . . . . . . . . . . . . . 19

2.3 Geometry-Based (CoV-Based) Regularity Metrics . . . . . . . . . . 22

2.3.1 The $\mathrm{CoV}$ of the Distances to the Nearest Neighbour . . . . . . 22

2.3.2 The CoV of the Areas of Voronoi Tessellation Cells . . . . . . 24

2.3.3 The $\mathrm{CoV}$ of the Lengths of Delaunay Triangulation Edges . . 24

2.4 Concluding Remarks . . . . . . . . . . . . . . . . . . 27

3 Hard-Core Point Processes for Modelling the Locations of Base $\begin{array}{ll}\text { Stations } & 29\end{array}$

3.1 Introduction . . . . . . . . . . . . . . . . . . . . . 29

3.1 .1 Contributions . . . . . . . . . . . . . . . . . 30

3.2 Hard-Core Point Processes . . . . . . . . . . . . . . . . . . . . 30

3.2.1 Matérn Hard-Core Process of Type I (MHC-I) . . . . . . . . . 32

3.2.2 Matérn Hard-Core Process of Type II (MHC-II) . . . . . . . . 32

3.2.3 Simple Sequential Inhibition (SSI) . . . . . . . . . . . . 33

3.3 Evaluation of CoV-based Metrics for Hard-Core Point Processes . . . 34

3.4 Comparison of $C_{\mathrm{N}}$ with two Function-Based Metrics . . . . . . . 37

3.4 .1 Ripley's K-Function . . . . . . . . . . . . . . . . . . 37

3.4 .2 Coverage Probability . . . . . . . . . . . . . . . 37

3.5 Concluding Remarks . . . . . . . . . . . . . . . . . . . . . 39

4 Perturbed Triangular Lattice for Modelling the Locations of Base Stations 41

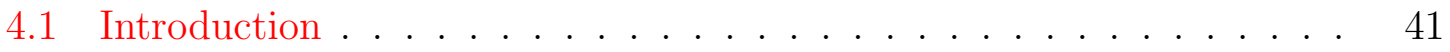


4.1 .1 Motivation . . . . . . . . . . . . . . . . . . . 42

4.1 .2 Contribution . . . . . . . . . . . . . . . . 42

4.2 Perturbed Triangular Lattices Models for Base Stations Placement . . 43

4.3 Measuring the Regularity of Perturbed Lattices _ . . . . . . . . . 45

4.4 Mapping Between Uniform PTL and Gaussian PTL Models Using the CoV-Based Metrics . . . . . . . . . . . . . . . . . 49

4.4 .1 Mapping . . . . . . . . . . . . . . . . . . . 49

4.4.2 Downlink Coverage Probability . . . . . . . . . . . . 49

4.5 Concluding Remarks . . . . . . . . . . . . . . . . . . . . 51

5 Towards the Best Metric for Measuring the Spatial Relationship among the Base Stations and Its Applications 55

5.1 Introduction . . . . . . . . . . . . . . 55

5.1 .1 Contribution . . . . . . . . . . . . . 57

5.2 Performance Metrics . . . . . . . . . . . . . . . . . . 58

5.2 .1 Downlink Coverage Probability . . . . . . . . . . . 58

5.2 .2 The Deployment Gain . . . . . . . . . . . . . . . . . 58

5.3 Strauss Point Process as Model for Base Station Placement _ . . . . 59

$5.3 .1 \quad$ Strauss Process . . . . . . . . . . . . . . . . . . . . 59

5.3.2 Measuring the Regularity of the Strauss Point Process Models 59

5.4 Quantifying the Deployment Gain Using the Regularity Metrics ... 61

5.4 .1 Data . . . . . . . . . . . . . . . . . . . 62

5.4 System Model . . . . . . . . . . . . . . . . . . . . 63

5.4 .3 Results. . . . . . . . . . . . . . . . . . . . . . 64

5.5 Fitting Base Station Locations to Repulsive Point Processes . . . . 69

5.6 The Heterogeneous Regularity of the Base Stations _ . . . . . . 73

5.7 Concluding Remarks . . . . . . . . . . . . . . . . 76

6 Cell Switch-Off for Networks Deployed With Variable Spatial Reg$\begin{array}{ll}\text { ularity } & 79\end{array}$

6.1 Introduction . . . . . . . . . . . . . . . . . . 79

6.1 .1 Motivation . . . . . . . . . . . . . . . . 80

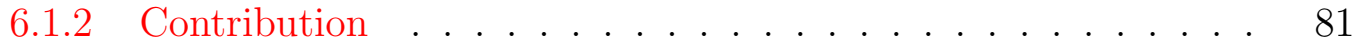

$6.2 \mathrm{CSO}$ as a Regularity Maximization Problem . . . . . . . . . . 82

6.2.1 The Problem Statement in Brief . . . . . . . . . . . . . . 82 
6.3 Proposed Algorithms for Cell Switch-Off . . . . . . . . . . . . 83

6.3.1 Greedy Construction (GC) . . . . . . . . . . . . . 84

6.3.2 Greedy Deletion (GD) . . . . . . . . . . . . . . 85

6.3.3 Semi-Greedy Deletion $(\mathrm{SG}) \ldots \ldots \ldots \ldots$

6.3.4 Random Switch-Off (RS) . . . . . . . . . . . . . . . 85

6.4 Downlink System Model … . . . . . . . . . . . . . . . 86

6.4 BSs Deployment . . . . . . . . . . . . . . . . 86

6.4 .2 Users Model . . . . . . . . . . . . . . . . . . . . . . . 86

6.4.3 LTE Urban Macro (UMa) Scenario . . . . . . . . . . . . . . 87

6.4.4 Simple Scenario . . . . . . . . . . . . . . . . . . . . . 87

6.5 Results . . . . . . . . . . . . . . . . . . . . . . . . 87

6.5 .1 SIR Gain . . . . . . . . . . . . . . . . . . . 88

6.5.2 Network Capacity . . . . . . . . . . . . . . . . . . . . . 91

6.5.3 Rate Coverage . . . . . . . . . . . . . . . . . . . . . . 91

6.5.4 Real BS Locations . . . . . . . . . . . . . . . . . . . . 91

6.6 Concluding Remarks . . . . . . . . . . . . . . . . . . . . . . . . 94

7 Strategic Densification with UAV-BSs in Cellular Networks $\quad 97$

7.1 Introduction . . . . . . . . . . . . . . . . . . 97

7.2 Contribution . . . . . . . . . . . . . . . . . . 98

7.3 Placement and Performance Metrics . . . . . . . . . . . . 100

7.3.1 Placement (Regularity) Metric . . . . . . . . . . . . . . 100

7.3.2 Performance Metrics . . . . . . . . . . . . . . . . . . . 100

7.4 Downlink System Model . . . . . . . . . . . . . . . . . . . . . . . . 101

7.5 Strategic Placement Method . . . . . . . . . . . . . . . . . . . 103

7.6 Results . . . . . . . . . . . . . . . . . . . . . . . . . 107

7.7 Concluding Remarks . . . . . . . . . . . . . . . . . . . . . . . 109

8 Conclusion and Future Work $\quad 115$

8.1 Summary and Conclusion . . . . . . . . . . . . . . 115

8.2 Potential Future Works . . . . . . . . . . . . . . . . . . . . . . . 118

8.2.1 New Hard-Core Models and CSO Algorithms . . . . . . . . . 119

8.2.2 Large-Scale UAV-BS Deployment in Wireless Cellular Networks with Spatially Heterogeneous Traffic Demand . . . . . . . 120 
List of References 


\section{List of Tables}

3.1 CoV-based metrics floor for hard-core point processes . . . . . . . . 36

4.1 Summary of the comparison between the matched uniform PTL and Gaussian PTL. . . . . . . . . . . . . . . . . 54

5.1 Summary of London Local Regularity information . . . . . . . . . . . 73 


\section{List of Figures}

1.1 Delaunay triangulation (golden dashed lines) and Voronoi tessellation (blue solid lines) for a spatial pattern (blue x marks) of a triangular lattice $(\mathrm{TL}) \ldots \ldots \ldots \ldots$. . . . . . . . . . . . . . . . . . . . 6

1.2 Delaunay triangulation (golden dashed lines) and Voronoi tessellation (blue solid lines) for a spatial pattern (blue x marks) of a homogeneous

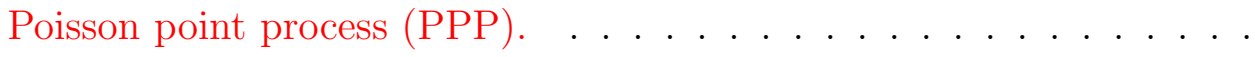

1.3 Delaunay triangulation (golden dashed lines) and Voronoi tessellation (blue solid lines) for a spatial pattern (blue $\mathrm{x}$ marks) of a repulsive point process $(\mathrm{RPP}) \ldots \ldots \ldots \ldots \ldots \ldots$

2.1 Probability density functions of two sets of data generated using two Gaussian random variables with mean, standard deviation, and $\mathrm{CoV}$ values $\left(\mu_{1}=10, \sigma_{1}=2.4, C_{1}=0.24\right)$ and $\left(\mu_{2}=100, \sigma_{2}=5, C_{2}=\right.$ 0.05), respectively. . . . . . . . . . . . . . .

2.2 An arbitrary spatial point pattern (blue " $\mathrm{x}$ " marks). The golden arrows indicate the distance from each point (at the arrow tail) to its nearest neighbour (at the arrowhead). Double-headed arrows means the points pair are the nearest neighbour of each other. . . . . . . . . . .

2.3 Delaunay triangulation (golden dashed lines) and Voronoi tessellation (blue solid lines) of a spatial pattern (blue plus marks) with different amounts of regularity as quantified using $C_{\mathrm{D}} \ldots \ldots . . \ldots 26$

3.1 The density ratio of MHC-I, MHC-II, and SSI as a function of $\tilde{r}$. . . .

3.2 The CoV of the areas of the Voronoi tessellation cells as a function of the normalized hard-core distance for hard-core point processes. . . .

3.3 The CoV of the lengths of the Delaunay triangulation edges as a function of the normalized hard-core distance for hard-core point processes.

3.4 The CoV of the distance to the nearest neighbour as a function of the normalized hard-core distance for hard-core point processes. . . . . . 
3.5 Normalized Ripley's K-function as a function of the hard-core distance $r$ (meters) for different RPPs and $C_{\mathrm{N}}$ values. . . . . . . . . 38

3.6 The coverage probability for different hard-core models with constant density for different $C_{\mathrm{N}}$ values and channel environments. . . . . . . .

4.1 The normalized $\mathrm{CoV}$ of the distance to the nearest neighbour as a function of the normalized perturbations. . . . . . . . . . .

4.2 The normalized $\mathrm{CoV}$ of the areas of the Voronoi tessellation cells as a function of the normalized perturbations. . . . . . . . . . . .

4.3 The normalized CoV of the lengths of the Delaunay triangulation edges as a function of the normalized perturbations. . . . . . . . . 47

4.4 The relationship between different CoV-based $\left(C_{\mathrm{N}}, C_{\mathrm{V}}\right.$, and $\left.C_{\mathrm{D}}\right)$ metrics of different PTL models. . . . . . . . . . . . . . . . . . . 48

4.5 The relationship between $\tilde{R}$ and $\tilde{\sigma}$ that gives equal $\mathrm{CoV}$-metrics value. 50

4.6 The coverage probability for different PTL models for different perturbation values and channel environments. . . . . . . . . . .

4.7 The difference in SIR between the curves of the coverage probability of different spatial patterns and the PPP coverage probability (the deployment gain). Path-loss model $\alpha=3$ and $X_{g}=6 \mathrm{~dB} \ldots \ldots$. .

4.8 The difference in SIR between the curves of the coverage probability of different spatial patterns and the PPP coverage probability (the deployment gain). Path-loss model $\alpha=4$ and $X_{g}=0 \mathrm{~dB}$. . . . . .

5.1 The normalized $\mathrm{CoV}$ of (a) the distance to the nearest neighbour $\left(C_{\mathrm{N}}\right)$, (b) the areas of the Voronoi tessellation cells $\left(C_{\mathrm{V}}\right)$, and (c) the lengths of the Delaunay triangulation edges $\left(C_{\mathrm{D}}\right)$ as a function of the interaction parameter and the normalized hard-core distance $\tilde{r}$ for Strauss

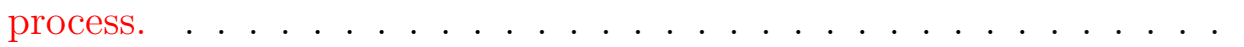

5.2 The relationship between the regularity as measured using the $C_{\mathrm{N}}$ metric and the deployment gain $G\left(p_{t}\right)$ at a target coverage probability $p_{t}=0.5$ of different simulated and actual deployments of BSs for different channel parameters: The path-loss exponent $\alpha$ and shadowing

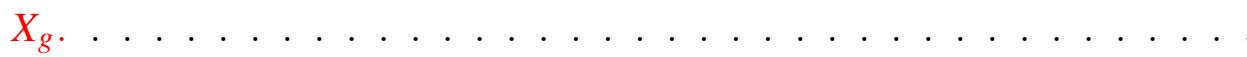


5.3 The relationship between the regularity as measured using the $C_{\mathrm{V}}$ metric and the deployment gain $G\left(p_{t}\right)$ at a target coverage probability $p_{t}=0.5$ of different simulated and actual deployments of BSs for different channel parameters: The path loss exponent $\alpha$ and shadowing $X_{g} \ldots \ldots \ldots \ldots \ldots \ldots \ldots \ldots \ldots \ldots$

5.4 The relationship between the regularity as measured using the $C_{\mathrm{D}}$ metric and the deployment gain $G\left(p_{t}\right)$ at a target coverage probability $p_{t}=0.5$ of different simulated and actual deployments of BSs for different channel parameters: The path-loss exponent $\alpha$ and shadowing $X_{g} \ldots \ldots \ldots \ldots \ldots \ldots \ldots \ldots \ldots \ldots \ldots \ldots \ldots \ldots \ldots$

5.5 Two similar (the amount of regularity $C_{\mathrm{D}}=0.75$ and density $\lambda=$ $38.73 \mathrm{BSs} / \mathrm{km}^{2}$ ) spatial deployments of BSs. (a) real (actual) data extracted from the Sitefinder database. It consists of 61 BSs distributed over $1500 \mathrm{~m} \times 1050 \mathrm{~m}$ region centred at $\left(51.5157^{\circ} \mathrm{N},-0.1395^{\circ} \mathrm{W}\right)$. (b) A realization using Gaussian PTL $(\tilde{\sigma}=0.3357) \ldots \ldots \ldots \ldots$. . . . .

5.6 Two similar (the amount of regularity $C_{\mathrm{D}}=0.38$ and density $\lambda=38.73$ $\mathrm{BSs} / \mathrm{km}^{2}$ ) spatial deployments of BSs. (a) Simulated data using SSI (hard-core distance $r=131.35 \mathrm{~m}$ ). (b) A realization using uniform PTL $(\tilde{R}=0.26) \ldots \ldots \ldots \ldots \ldots \ldots \ldots$

5.7 The BSs belong to the Vodafone operator. The regions are (a) $73.5 \mathrm{~km} \times 73.5 \mathrm{~km}$, (b) $10.5 \mathrm{~km} \times 10.5 \mathrm{~km}$, and (c) $1.5 \mathrm{~km} \times 1.5 \mathrm{~km}$. They are centred at $\left(51.5136^{\circ} \mathrm{N}, 0.1342^{\circ} \mathrm{W}\right)$, London, UK. The smallscale region is an enlarged version of the central area (golden square) of the medium-scale region, which itself is an enlarged version of the central area (red square) of the large-scale region. . . . . . . . . . .

6.1 Network deployment regularity before $\left(C_{\mathrm{D}}(\mathrm{in})\right)$ and after $\left(C_{\mathrm{D}}\right.$ (out)) applying the CSO algorithms at different CSO percentages $\rho . \ldots$.

6.2 The SIR gain $G_{\mathrm{p}}(0.5)$ as a function of the network deployment regularity before the CSO $\left(C_{\mathrm{D}}(\mathrm{in})\right)$ at different CSO percentages $\rho . .$.

6.3 Normalized network capacity for LTE UMa scenario as a function of the CSO percentages $\rho$ for two different network deployment in terms of regularity. The regularity maximization gain is annotated by thick red line $(\mathrm{b}) \ldots \ldots \ldots \ldots \ldots \ldots \ldots \ldots \ldots \ldots$ 
6.4 Rate coverage for LTE UMa scenario as a function of the CSO percentages $\rho$ for two different network deployment in terms of regularity.

6.5 Applying the greedy deletion (GD) algorithm on real BS locations at different CSO percentages $\rho$. Blue and golden towers represent the active and inactive BSs, respectively. The BSs (GSM band $900 \mathrm{MHz}$ ) belong to the Vodafone operator. The region is $1500 \mathrm{~m} \times 1050 \mathrm{~m}$, in London, UK, centred at $\left(51.5136^{\circ} \mathrm{N}, 0.1342^{\circ} \mathrm{W}\right)$. This data is similar to that used in $[13$, Fig. 1] . . . . . . . . . . . . . . . .

6.6 Applying the semi-greedy deletion $(\mathrm{SG})$ algorithm on real BS locations at different CSO percentages $\rho$. Blue and golden towers represent the active and inactive BSs, respectively. The BSs (GSM band $900 \mathrm{MHz}$ ) belong to the Vodafone operator. The region is $1500 \mathrm{~m} \times 1050 \mathrm{~m}$, in London, UK, centred at $\left(51.5136^{\circ} \mathrm{N}, 0.1342^{\circ} \mathrm{W}\right)$. This data is similar to that used in $[13$, Fig. 1] . . . . . . . . . . . . . . . . .

7.1 Graphical illustration of the strategic deployment of UAV-BSs in cellular networks. . . . . . . . . . . . . . . . . . .

7.2 (a) Arbitrary T-BSs deployment (blue towers) with random candidate location (red dots). (b) T-BSs with the projection of the UAV-BSs on the ground (red disks) as selected from the random candidates using Algorithm 1. . . . . . . . . . . . . . . . . 106

7.3 The $C_{\mathrm{D}}$ (out) of the network as a function of the augmentation percentage $\zeta$. Each sub-figure represents a specific regularity value $C_{\mathrm{D}}$ (in) of the original network deployment (similar to [59, Fig. 1]) . . . . . . .

7.4 The median SINR as a function of the augmentation percentage $\zeta$ for different network deployment regularity $C_{\mathrm{D}}($ in) $\ldots . . . . . .111$

7.5 Normalized network capacity as a function of the UAV-BS augmentation percentages $\zeta$ for different network deployment regularity $C_{\mathrm{D}}$ (in). 112

8.1 Graphical illustration of UAV-BS deployment in a wireless cellular networks with spatially heterogeneous user distribution. . . . . . . . . 120 


\section{Nomenclature}

\begin{tabular}{ll} 
Acronym & Meaning \\
\hline BS & base station \\
CCDF & complementary cumulative distribution function \\
CDF & cumulative distribution function \\
CoV & coefficient of variation \\
CSO & cell switch-off \\
DPP & detrimental point processes \\
GC & greedy construction heuristic \\
GD & greedy deletion heuristic \\
HetNets & heterogeneous networks \\
LTE & long term evolution \\
MHC-I & Matérn hard-core process of type I \\
MHC-II & Matérn hard-core process of type II \\
PHC & Poisson hard-core process \\
PPP & Poisson point process \\
PTL & perturbed triangular lattice \\
RPP & repulsive point process \\
RS & random switch-off \\
SG & semi-greedy deletion heuristic \\
SINR & signal-to-interference-plus-noise ratio \\
SIR & signal-to-interference ratio \\
SSI & simple sequential inhibition \\
T-BS & terrestrial base station \\
TL & triangular lattice \\
UAV-BS & unmanned aerial vehicle mounted base station \\
UMa & urban macro \\
\hline
\end{tabular}




\section{List of Symbols}

\begin{tabular}{|c|c|}
\hline Symbol & Meaning \\
\hline$\sigma$ & the standard deviation \\
\hline$\mu$ & the arithmetic mean \\
\hline$C$ & the coefficient of variation $(\mathrm{CoV})$ \\
\hline$C_{\mathrm{N}}$ & The CoV of the the distances to the nearest neighbour \\
\hline$C_{\mathrm{V}}$ & The CoV of the areas of the cells of Voronoi tessellation \\
\hline$C_{\mathrm{D}}$ & The CoV of the length of the edges of Delaunay triangulation \\
\hline$\mu_{\mathrm{N}}$ & the mean of of the nearest neighbour distances \\
\hline$\sigma_{\mathrm{N}}$ & the standard deviation of the nearest neighbour distances \\
\hline$k_{\mathrm{N}}$ & a normalization factor \\
\hline$C_{i}$ & Voronoi cell \\
\hline$x_{i}$ & a point belongs to a spatial point pattern $\mathrm{X}$ \\
\hline $\mathrm{X}$ & a spatial point pattern \\
\hline$n(X)$ or $|X|$ & the number of the points in a a spatial point pattern $\mathrm{X}$ \\
\hline$\|\cdot\|$ & the Euclidean distance \\
\hline$k_{\mathrm{V}}$ & a normalization factor \\
\hline$\mu_{\mathrm{V}}$ & the mean of the Voronoi cell areas \\
\hline$\sigma_{\mathrm{V}}$ & standard deviation of the Voronoi cell areas \\
\hline$\mu_{\mathrm{D}}$ & the mean of the Delaunay edge lengths \\
\hline$k_{\mathrm{D}}$ & a normalization factor \\
\hline$\sigma_{\mathrm{D}}$ & the standard deviation of the Delaunay edge lengths \\
\hline$\lambda$ & the density of a point process \\
\hline$r$ & hard-core distance \\
\hline$\tilde{r}$ & normalized hard-core distance \\
\hline$\Phi$ & Poisson point process \\
\hline$\lambda_{\mathrm{P}}$ & the primary density of a point process \\
\hline$B\left(x_{i}, r\right)$ & is a ball with centre $x_{i}$ and radius $r$ \\
\hline$\pi$ & $\approx 3.14159$ \\
\hline$K(r)$ & the Ripley's K-function \\
\hline
\end{tabular}




\section{List of Symbols}

\begin{tabular}{ll} 
Symbol & Meaning \\
\hline$L(r)$ & the L-function \\
$\alpha$ & a path loss exponent \\
$X_{g}$ & a lognormal shadowing \\
$G$ & a generator matrix \\
$\eta$ & the distance between any two nearest neighbour points in a TL \\
$R$ & the perturbation distance of the uniform PTL \\
$\tilde{R}$ & the normalized perturbation distance of the uniform PTL \\
$\sigma$ & the perturbation distance (the standard deviation) of the Gaussian PTL \\
$\tilde{\sigma}$ & the normalized perturbation distance of the Gaussian PTL \\
$P(\gamma)$ & the downlink coverage probability \\
$G\left(p_{t}\right)$ & the deployment gain \\
$p_{t}$ & the target coverage probability \\
$\bar{F}_{\mathrm{SIR}}^{-1}$ & the inverse of the CCDF of the SIR \\
$s(\mathrm{X})$ & the number of point pairs that are closer than a hard-core distance $r$ apart \\
$\Gamma_{\mathrm{D}}$ & the regularity gain \\
$C_{\mathrm{D}}^{\text {ref }}$ & the $C_{\mathrm{D}}$ of a reference model \\
$\rho$ & the CSO percentage \\
$d_{i, j}$ & the distance between the points $i$ and $j$ \\
$\operatorname{dist}\left(x_{i}, x_{j}\right)$ & the euclidean distance between the points $x_{i}$ and $x_{j}$ \\
$\operatorname{dist}\left(x_{j}, \mathcal{B}\right)$ & the distance between the point $x_{j}$ and its nearest neighbor point in the set $\mathcal{B}$ \\
$\theta_{i j}$ & the elevation angle (degrees) between a user $i$ and UAV-BS $j$ \\
$\phi_{i j}$ & $=90-\theta_{i j}$ \\
$\mathbb{P}_{\mathrm{LoS}}$ & the occurrence probability of the LoS link \\
$\mathbb{P}_{\mathrm{NLoS}}$ & the occurrence probability of the NLoS link \\
$\zeta$ & the percentage of the augmented UAV-BSs to the T-BSs \\
$\mathcal{A} \| C$ & the concatenation of the sets $\mathcal{A}$ and $C$ \\
$h_{j}$ & the altitude of a UAV-BS $j$ \\
$R_{j}$ & the radius of the coverage region of a UAV-BS $j$ \\
\hline &
\end{tabular}




\section{Chapter 1}

\section{Introduction}

"A man is a scholar as long as he continues learning, but if he stops learning and thought that he has achieved enough knowledge, then he becomes the most ignorant."

—Sa'id ibn Jubayr

\subsection{Motivation}

The proliferation of the forefront mobile broadband communication devices such as smartphones, laptops, and tablets as well as the most recent virtual and augmented reality devices has led the cellular networks to leapfrog beyond their original design of voice-centric to data-centric applications, e.g., live Internet video streaming and broadcasting. Unsurprisingly, mobile data traffic is growing strongly year-on-year [1] because of this paradigm shift. In the near future, the traffic of wireless and mobile devices is expected to surpass the traffic of all wired devices, and it will probably

account for more than two-thirds of total data traffic by 2021 [2]. The challenge of mobile data growth urges the cellular industry to prepare for 1000x fold data increase every decade [3].

Among several approaches to cope with this data deluge, deploying more base stations (BSs) has been recognized as a key solution, which brings the network 
closer to the user and results in reducing the signal attenuation and increasing the line-of-sight probability. Clearly, all the performance metrics in cellular networks such as signal-to-interference-(plus-noise) ratio $(\mathrm{SI}(\mathrm{N}) \mathrm{R})$, coverage probability, and capacity/throughput/data-rate, rely on the spatial structure of the base stations, i.e., depend on the distances between the transmitters and receivers. Therefore, the increase in the number of BSs necessitates modelling and characterizing the spatial configuration of the network. This leads to fully understand the relationship between the spatial deployment of the BSs and fundamental cellular network quality metrics [4] as well as to design and plan future cellular networks. Consequently, modelling and analyzing the placement of BSs in wireless networks have drawn the attention of the researchers in both industry and academia in recent years.

Fortunately, a mature field of mathematics called stochastic geometry is ready to facilitate understanding these relationships. Stochastic geometry studies the random statistical spatial patterns, i.e., random geometric shapes and random distribution of points. It provides tremendous sophisticated mathematical tools such as spatial point processes, random tessellations, and summary characteristics [4-6] that can be used for the analysis of the wireless networks..

In the last few years, stochastic geometry has been extensively used for the analysis of wireless cellular networks, yet it is still a new practise in the field. For example, it has been used for modelling the network structure, fitting a real deployment of BSs to a model, and quantifying the spatial structure of the network. Stochastic geometry is the primary mathematical tool that is used in this thesis.

Various point processes have been investigated to model the spatial structure of the BSs. The current literature indicates that there is no consensus among the researchers on which point process is the most suitable or useful to mode BS locations: researchers have different perspectives and expectations for the model they advocate. 
Indeed, a desirable point process model has four properties: 1) realistic, 2) tunable for the full range of regularity with few controlling parameters, 3) analytically tractable, 4) computationally inexpensive and straightforward to simulate. Interestingly, it is hard to find a model that has all these properties. For example, while the PPP is superior in the tractability and simple to simulate, it is neither realistic nor tunable. Investigating the suitability of different point processes to model the deployment of the BSs is still a rich research realm.

Moreover, the amount of spatial relationship (or, equivalently, the amount/degree of regularity/irregularity/repulsiveness) of a pattern of the spatial structure of a set of BSs is a fundamental factor that affects the performance of a cellular network. In fact, quantifying the amount of regularity for a set of BSs is vital to facilitate the understanding and interpreting the relationship between the spatial structure and the performance of a network. This understanding leads to better designing and planning of next-generation cellular networks as we see in this thesis.

Furthermore, the regularity/irregularity degree of a pattern of the spatial structure of a set of BSs is a fundamental factor that affects the performance of a cellular network, yet there is no precise and meaningful metric to describe it. In fact, quantifying the amount of regularity (repulsiveness) for a set of BSs is vital to facilitate the understanding and the interpretation of the relationship between the spatial structure and the performance of a network. This understanding leads to a better designing and planning for the next-generation cellular networks. Noteworthy, no prior work has explored quantifying the regularity of the BSs in cellular networks using scalar metrics. Finding a good scalar metric to quantify the regularity is a necessity; it is, in fact, an interesting niche topic of research.

As mentioned before, the intense deployment of BSs is an important technique to 
meet the growth of the data demand. Noticeably, the distribution of the traffic demand fluctuates during the day, yet cellular networks are usually designed to handle the peak-hour traffic. Such an over-provisioning leads to a waste of capacity, energy, and capital expenditure. The infrastructure resources of the network should be utilized efficiently. To address the uneven traffic distribution, this thesis investigates two possible approaches: the cells switch-off (CSO) during the low traffic periods, or deploying mobile on-demand BSs, specifically, BSs mounted on unmanned aerial vehicles (UAV-BSs). While the CSO approach reduces the operational expenditure, the UAV-BSs approach reduces the capital expenditure.

\section{$1.2 \quad$ Literature Review}

To put this work in perspective, in this section we provide a brief overview of the point processes and their use as models for the BS locations. We note that while a plethora of models is available, there is no consensus on which model to use. More research is indispensable to find better models.

We also provide bird's-eye views of methods and tools for fitting mathematical models to actual spatial point patterns. Notably, these methods and tools are represented as functions, which we refer to as function-based metrics, and the literature lacks scalar metrics to be used to test the fitness and to measure the spatial relationship between the points.

\subsubsection{Point Process Models for Base Station Locations}

Point processes can be classified into three categories: the completely spatial random point process (i.e., PPP), the repulsive point processes (RPPs), and the clustered point processes. Roughly speaking, the points of a repulsive point process 
exhibit repulsion between each other and are used to model the BS locations, while the points of a clustered point process attract each other in the form of groups and are more suitable to model the user locations and machine clusters [7]. In this thesis, we confine our effort to the use of the RPPs for modelling the BS locations since they are the most appropriate models.

Recent years have shown a trend in testing point processes for modelling the base station locations in cellular networks. Before considering the random point processes, the deterministic triangular lattice (TL) placement (i.e., hexagonal layout, where their coverage regions appeared as hexagons, as shown in Figure 1.1) had been the most popular model both in industry and academia to model the location of the BSs. The cellular networks per se gained their name from this model. It has been found in industry standards and used mainly for the system level simulations due to its simplicity. However, the deployment of the BSs in real networks does not form a perfect hexagonal layout due to the several natural and human-made reasons such as the restrictions in the site acquisition, radio propagation environment, landscape, terrain, topography, bodies of water, population distribution, and capacity demand [8]. These factors prevent the network operators from placing the BSs in the wanted locations, which results in an irregular network topology.

Owing to its analytical tractability, an entirely spatially independent random placement (i.e., deploying BSs according to a PPP, as illustrated in Figure 1.2) is widely accepted nowadays for modelling cellular networks instead of the traditional TL (hexagonal layout) placement. Although this BS modelling approach has been proposed for almost two decades [9,10], the well-cited article by Andrews et al. [4] and its extension for heterogeneous networks by Dhillon et al. [11] brought much attention to modelling cellular network using PPP which later led to extensive use 


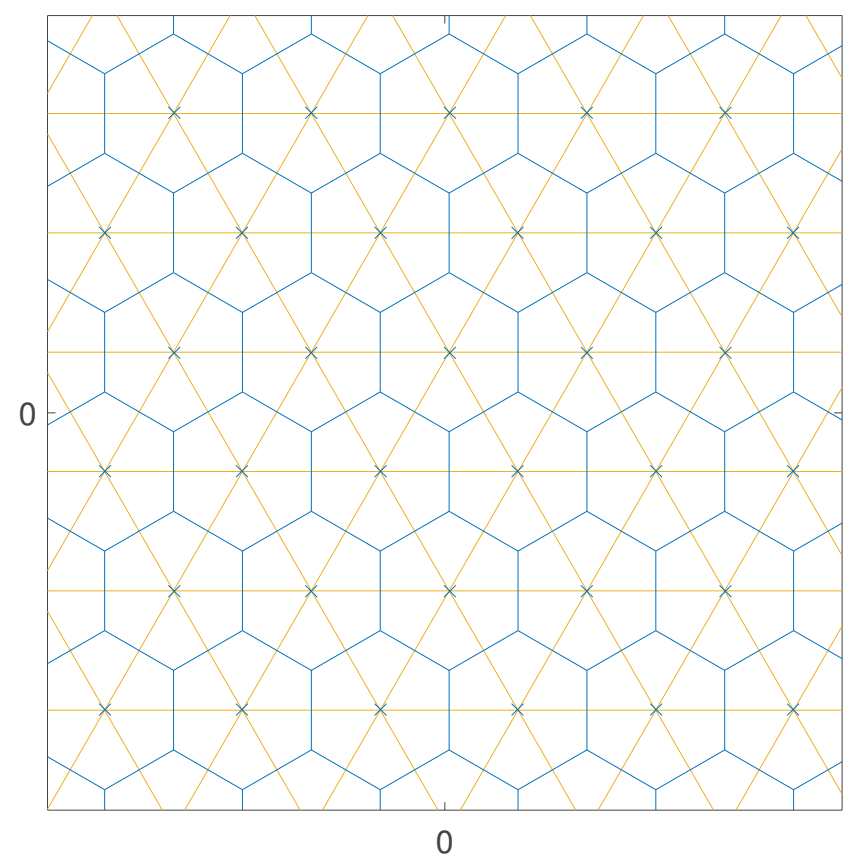

Figure 1.1: Delaunay triangulation (golden dashed lines) and Voronoi tessellation (blue solid lines) for a spatial pattern (blue x marks) of a triangular lattice (TL).

of the stochastic geometry tools in cellular networks. Nevertheless, this model is not accurate since BSs are not arbitrarily placed in the real networks. The network operators spread out the BSs to the maximum possible extent during the planning phase to reduce the interference.

That being said, it is evident that both the TL and the PPP are unrealistic models and represent two extremes. While the TL model fails to capture the irregularity of the real networks, the PPP approach fails to capture the spatial correlation between the BSs. While modelling BS locations using a TL gives over-optimistic network performance results, modelling BS locations using the PPP gives the most pessimistic performance estimate [12]. The real deployment of the BS locations falls somewhere in between [12-15]. Notably, both models are conceptually too simple, and they can be characterized by a single parameter: the density. 


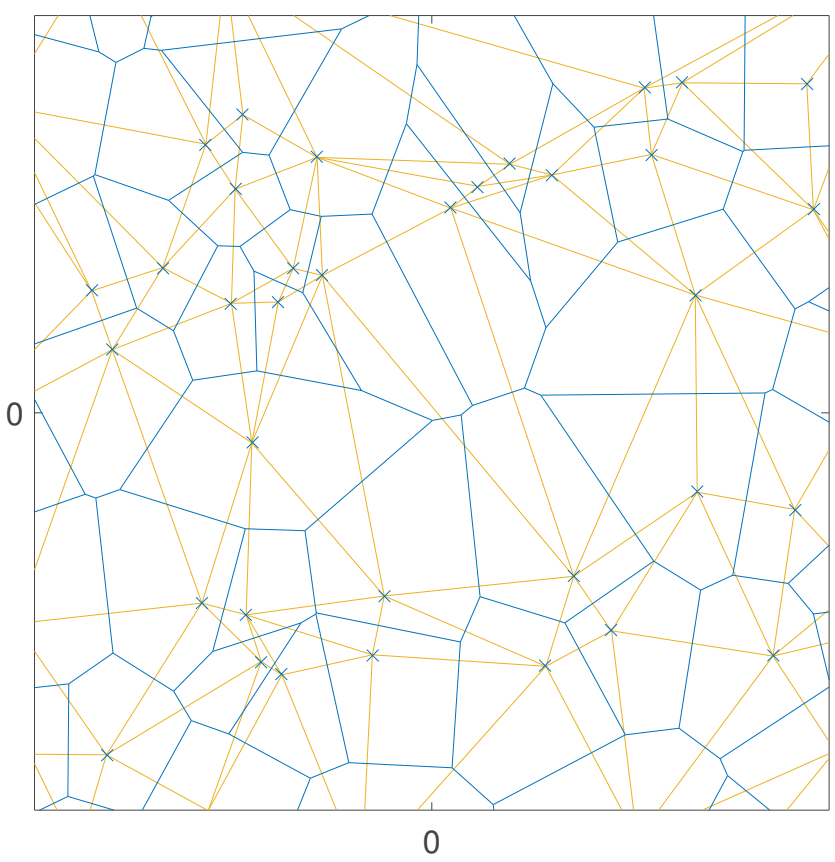

Figure 1.2: Delaunay triangulation (golden dashed lines) and Voronoi tessellation (blue solid lines) for a spatial pattern (blue x marks) of a homogeneous Poisson point process (PPP).

Because of their regularity property (more regular than a PPP, i.e., capable of generating spatial patterns that lie between TL and PPP), researchers have enthusiastically embraced repulsive point processes (RPPs) to realistically model and analyze BSs placements in cellular networks. A repulsive point process (RPP) - also called a regular point process or, equivalently, a sub-Poisson point process - has a spatial pattern whose points appear to repel each other as shown, for example, in Figure 1.3. As the repelling force increases, the regularity of the spatial pattern of the points increases.

RPPs have two varieties: soft-core processes and hard-core processes. While a hard-core process strictly prohibits its points from being closer than a predefined hard-core distance, a soft-core process allows its points to appear arbitrarily close to each other with a certain probability and rules. The soft-core variety of RPPs that are 


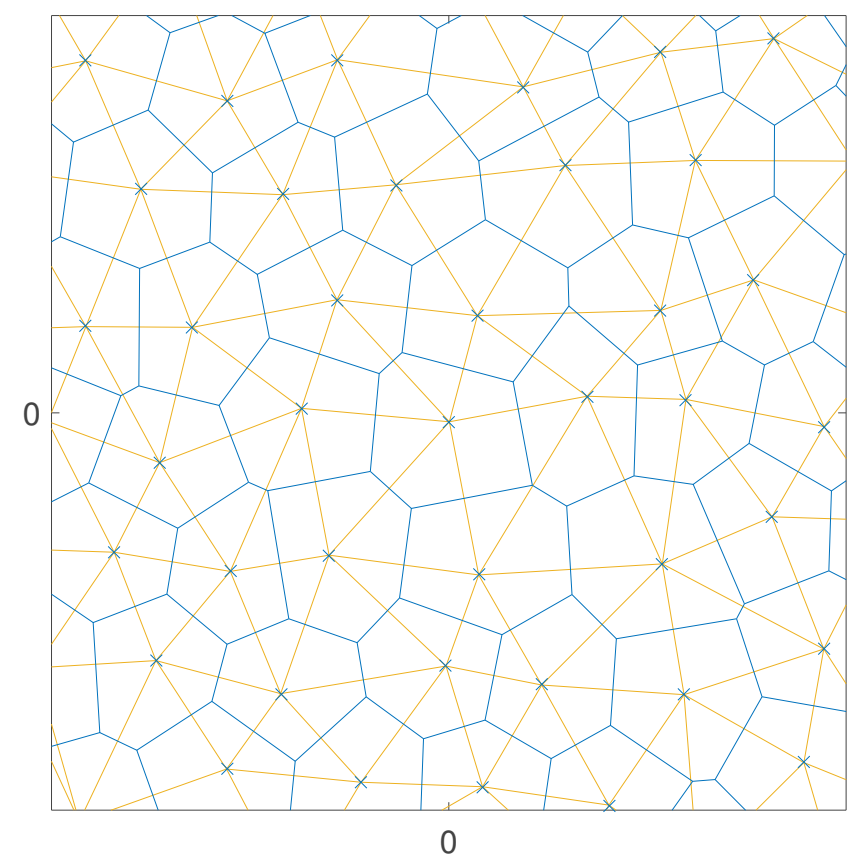

Figure 1.3: Delaunay triangulation (golden dashed lines) and Voronoi tessellation (blue solid lines) for a spatial pattern (blue $\mathrm{x}$ marks) of a repulsive point process (RPP).

used for modelling the access points (e.g., BS locations) in wireless networks includes 1) the determinantal point process models [16-20], and 2) the family of Gibbs point processes $[13,15,21]$. The hard-core variety of RPPs includes the Matérn hard-core processes of type I and type II [5,22], the simple sequential inhibition (SSI) process [5], and Poisson hard-core (PHC) process.

It is worth noting that the generation of all these RPPs is initiated by generating entirely random spatially independent points, and then deleting specific points based on some formula involving their mutual distances and sometimes their sequential order. The generation methods are computationally expensive since it is also often necessary to generate many points and calculate the distance between each pair to select a smaller set of points as the output. Moreover, these RPPs have limited ability to provide a very high degree of regularity [13,23], as it is impossible to attain a TL by removing points from a PPP, for instance. (Removing points for a point process 
is called thinning.)

\subsubsection{Fitting Methods and Tools}

A growing body of literature is dedicated to investigating fitting actual BS locations to point processes. Li et al., [16] tested the fitness of actual real-world BS deployments to three different Detrimental point processes (DPP): The Gauss model, the Cauchy model, and the generalized gamma model. In terms of coverage probability, the generalized gamma model provides the best fit because of its high repulsiveness, and, in contrast, Gauss model provides the less precise fit because of its low repulsiveness. In [17], Ginibre point process — another significant example of DPP models - is accurately fitted to some real BS deployments.

Many functional summary characteristics (e.g., the nearest neighbour distribution function, and the empty space function as well as the Ripley's K-function and its normalizations the J- and L-functions) have been used to test the fitness of the proposed models to real BS deployments $[13,15-17,21]$. They are classical stochastic geometry metrics. Besides, these tools have been used to describe the spatial structure of the BSs and to measure the spatial relationship between them. However, they are impractical, especially for the latter purpose because they are function-based metrics that need the addition of further scalar metrics or qualitative terms such as less (or more) repulsive/regular (or clustered) to compare the different spatial pattern of points. It is desirable to have a scalar metric at the first place.

The current wireless literature made some attempts regarding proposing scalar metrics to measure the spatial relationship between the nodes. For example, Ganti and Haenggi [24] proposed using the noise figure and the variance of nearest neighbour distance to quantify the regularity of repulsive point process in the context of wireless sensor networks. Besides, Mirahsan et al., [25-27] proposed using the coefficient of 
variation $(\mathrm{CoV})$ of particular random spatial properties - the CoV of the the distances to the nearest neighbour $\left(C_{\mathrm{N}}\right)$, the $\mathrm{CoV}$ of the areas of the cells of Voronoi tessellation $\left(C_{\mathrm{V}}\right)$, and the $\mathrm{CoV}$ of the length of the edges of Delaunay triangulation $\left(C_{\mathrm{D}}\right)$ - to capture the spatial structure of the users modelled using clustered point processes. In spite of the good qualitative understanding of the spatial relationship of the patterns formed by the point processes [5,28] (e.g., the classification into clustered, entirely spatially random, and repulsive point processes as well as lattices), it is apparent that there is no comprehensive study on comparing the amount of spatial relationship of two point processes as models for BSs deployment in a quantified way.

\subsection{Scope and Objectives}

The focus of this thesis is to describe the spatial structure of the BSs of any cellular network only using two scalar measures: the density of the BSs and the quantity of regularity. We intend to demonstrate that these two measures are much more important than the type of the point process used to produce or model the spatial pattern. Knowing the density and the amount of regularity allows comparing different point processes regardless of their internal parameters. In addition, it permits fitting real spatial patterns to models in a novel way. This thesis aims to hide the model type and its internal parameters under the density and the amount of regularity of the produced spatial pattern. This abstraction is even more useful if the point process has three or more internal parameters. Proposing scalar metrics to quantify the spatial relationship is a prerequisite to find a qualified model, to obtain and efficient fitting procedure, to estimate the network performance, and to design the next-generation networks. This thesis endeavours to answer the following important questions:

1. What is the most robust scalar metric to adequately quantify the regularity and 
accurately capture the network performance?

2. What is the most suitable and useful point process to model the BSs deployment?

3. Is it possible to use the proposed metrics to adjust the internal parameters of different point processes to generate similar spatial patterns?

4. Is it possible to use the proposed metrics to fit a point process an actual deployment of BSs?

5. Given that the performance and the amount of spatial regularity are known for a network, is it possible to estimate the performance of another network that has the same number of BSs and network parameters, but has a different amount of spatial regularity based on the spatial regularity difference?

6. How to use the answers to the above questions toward designing and planning the agile future wireless networks?

\subsection{Contributions}

This thesis fills the void in the literature by answering the research questions posed above. The main contributions are summarized as follows:

\subsubsection{Proposing Scalar Metrics to Measure the Regularity (Irregularity)}

We proposed and examine the use of the $\mathrm{CoV}^{1}$ of three random geometric properties - the area of the cells of Voronoi tessellation, the length of the edges of Delaunay

\footnotetext{
${ }^{1}$ The concept of the CoV-based metrics is explained in Chapter 2.
} 
triangulation, and the distances to the nearest neighbour - of a set of points (BS locations) as metrics to quantify the regularity of a repulsive BS deployment. The results show that the $\mathrm{CoV}$ of the length of the edges of Delaunay triangulation $\left(C_{\mathrm{D}}\right)$ is the best metric for measuring the amount of regularity of RPPs, for indicating the network performance, and for fitting models to spatial point patterns.

\subsubsection{Advocating Perturbed Triangular Lattice (PTL) Model}

1. We investigate the suitability of three hard-core point processes (MHC-I, MHCII, and SSI) regarding their regularity range and the density of the generated points as models for deployment of BSs . The results indicate that the SSI point process is the most attractive among these hard-core point processes because of its wide regularity range compared to the other two, while MHC-I is the worst. However, these point processes are computationally expensive and unable to generate point patterns with very high regularity.

2. We advocate the use of the PTL to model the BS locations because it overcomes the limitations of the hard-core point processes and all other RPPs. The PTL is tunable over the whole range between the deterministic TL and the PPP, and it is computationally inexpensive. Moreover, it is simple and more realistic.

\subsubsection{Creating a Novel Framework for Mapping between Point Processes}

We provide a systematic approach to adjust the internal parameters of different point processes to produce spatial patterns with the same amount of regularity, and to check whether two given spatial patterns are similar or not. 


\subsubsection{Proposing a New Approach for Approximating the Per- formance of the Repulsive Cellular Networks}

Given a performance of a reference model (e.g., PPP-deployed network), we propose a simple approach to approximate the performance of different repulsive deployments of the BSs. In particular, we use the amount of regularity (or, specifically, the difference in regularity between) of two spatial sets of BSs to estimate the performance of the second set if the performance of the first set is known. The amount of regularity is used to compare the coverage performance of different spatial deployments. We presented a simple formula to convert the amount of regularity to average deployment gain. $^{2}$ Indeed, we demonstrate the simplicity, practicality, and effectiveness of our approach to carrying the results of one point process into another.

\subsubsection{Proposing a New Approach for Fitting Point Processes to BSs}

We propose a novel approach for fitting RPP models to actual BS deployments. Given a real deployment of BSs, we use the CoV of the length of the edges of Delaunay triangulation $\left(C_{\mathrm{D}}\right)$ as a metric to identify how to adjust the internal parameters of a particular point process to generate a spatial pattern equivalent to the real deployment.

\subsubsection{Devising a Novel Energy Saving Approach}

We identify and address a novel CSO problem, which is based on switching off some BSs to save energy during low-traffic periods. This CSO problem relies on the understanding of the relationship between the spatial regularity of the BSs and

\footnotetext{
${ }^{2}$ The concept of the deployment gain is explained in Chapter 5 .
} 
the network performance. That is to say, it is based on maximizing the network's performance through optimizing the spatial regularity of the remaining active BSs. This work is the first to consider repulsive BS deployment in the CSO literature.

\subsubsection{Devising Novel Strategies for Large-Scale UAV-BSs Placement}

We leverage the previous contributions to propose a novel approach for large-scale integration of UAV-BSs with terrestrial networks. We present a strategic densification method that is empowered by the stochastic geometry and the optimization tools to provide the most appropriate placement for these UAV-BSs. We also provides supplyside estimation for number UAV-BSs that are needed to densify a terrestrial network such that it reaches a targeted network performance benchmark.

\subsection{Publications}

The following is a list of the publications pertaining to this thesis and produced during the PhD program enrolment.

\subsubsection{Journal Papers - Included in the Thesis}

- [J4] Faraj Lagum, Sebastian Szyszkowicz, and Halim Yanikomeroglu, "A metric for measuring the spatial relationship among the base stations and its applications," under review in IEEE Transactions on Wireless Communications. (Submission: 06 September 2018.)

- [J3] Faraj Lagum, Irem Bor-Yaliniz, and Halim Yanikomeroglu, "Strategic densification with UAV-BSs for cellular networks," IEEE Wireless Communications 
Letters, vol. 7, no. 3, pp. 384-387, June 2018.

- [J2] Faraj Lagum, Quoc-Nam Le-The, Tamer Beitelmal, Sebastian S. Szyszkowicz, and Halim Yanikomeroglu, "Cell switch-off for networks deployed with variable spatial regularity," IEEE Wireless Communications Letters, vol. 6, no. 2, pp. 234-237, April 2017.

- [J1] Faraj Lagum, Sebastian S. Szyszkowicz, and Halim Yanikomeroglu, "CoVbased metrics for quantifying the regularity of hard-core point processes for modeling base station locations," IEEE Wireless Communications Letters, vol. 5, no. 3, pp. 276-279, June 2016.

\subsubsection{Conference Paper - Included in the Thesis}

- [C1] Faraj Lagum, Sebastian S. Szyszkowicz, and Halim Yanikomeroglu "Quantifying the regularity of perturbed triangular lattices using CoV-based metrics for modeling the locations of base stations in HetNets," IEEE 84th Vehicular Technology Conference (VTC-Fall), Montreal, QC, 2016, pp. 1-5.

\subsubsection{Journal Papers - Not Included in the Thesis}

- [J2] Mohamed Alzenad, Amr El-Keyi, Faraj Lagum, Halim Yanikomeroglu, "3-D Placement of an unmanned aerial vehicle base station (UAV-BS) for energy-efficient maximal coverage," IEEE Wireless Communications Letters, vol. 6, no. 4, pp. 434-437, Aug. 2017.

- [J1] Quoc-Nam Le-The, Tamer Beitelmal, Faraj Lagum, Sebastian S. Szyszkowicz, Halim Yanikomeroglu, "Cell switch-off algorithms for spatially irregular base station deployments," IEEE Wireless Communications Letters, 
vol. 6, no. 3, pp. 354-357, June 2017.

\subsection{Organization of the Thesis}

The remainder of the thesis is divided into two parts: theoretical investigations (Chapter 2, Chapter 3, Chapter 4, and Chapter 5) and practical applications (Chapter 6 and Chapter 7). In Chapter 2, we explore three stochastic geometry properties of a set of points to use them as measures of the spatial relationship. In Chapter 3, we apply these geometry-based metrics to three hard-core point processes to test their suitability for BS modelling. In Chapter 4, we advocate the PTL to model the BS locations and present how to use the regularity metrics to map the parameter of one spatial model to another. In Chapter 5, we show that the $\mathrm{CoV}$ of the length of the edges of Delaunay triangulation is the best metric for measuring the spatial regularity of the BSs and capturing the network performance. Using this metric, we propose simple novel approaches to approximate the network performance and fit repulsive point processes to BS locations. Chapter 6 and Chapter 7 leverage the results of the three previous chapters by presenting two novel and practical use cases for the regularity metrics. While Chapter 6 presents a CSO approach, Chapter 7 presents a variety of UAV-BSs placement strategies. Finally, a conclusion and a potential future research directions are presented in Chapter 8. 


\section{Theoretical Investigations}




\section{Chapter 2}

\section{Geometry-Based Scalar Metrics to Quantify the Spatial Relationship among the Locations of the Base Stations}

"If you can't measure it, you can't improve it."

- Peter Drucker

\section{$2.1 \quad$ Introduction}

Due to the dependence of the network performance on the BS locations [13], the amount of spatial relationship (or, equivalently, the amount/degree of regularity/irregularity/repulsiveness) of the spatial structure of BSs is an important characteristic of wireless cellular networks. Motivated by the lack of an adequate scalar metric to describe the spatial structure of these BSs, we propose using the coefficient of variation $(\mathrm{CoV})$ of particular geometric properties of the BS locations as scalar metrics to quantify their regularity [25-27]. (We call these metrics the geometry-based or, equivalently, the CoV-based regularity metrics.) These scalar metrics are useful as network performance indicators as shown in the next chapters. Functional summary characteristics such as the nearest neighbour distribution function, the empty space function, and the Ripley's K-function are widely used in the literature [13,15-17,21,29] 
as metrics to capture the spatial structure of wireless networks. However, these metrics are functions, and therefore we still need to quantify the difference between them. Qualitative terms such as less (or more) repulsive [5, 17, 24, 30] are also used in the literature to compare the amount of regularity of RPPs. Therefore, finding a precise and meaningful scalar metric to quantify the spatial regularity is a necessity [24].

In this chapter, we introduce three spatial properties of a set of points that can be used to quantify the spatial relationship among the points of the set (measure the regularity of the $\mathrm{BSs}$ ), which are the distances to the nearest neighbour, the areas of the cells of Voronoi tessellation, and the edges of Delaunay triangulation. In Chapter 3, Chapter 4, Chapter 5, we examine these spatial properties to find the best among them for capturing the spatial structure of the BSs.

\subsection{The Coefficient of Variation}

The coefficient of variation $(\mathrm{CoV})$ of a random variable or set of scores (i.e., quantities or numbers) of a data is defined as the ratio of its standard deviation to its mean. The $\mathrm{CoV}$ is an important measure of variability, spread, or dispersion. It is a unitless alternative to the other measures of variability such as the variance and the standard deviation [31,32]. Therefore, it is appropriate to compare the measurements from different observations regardless of their units. Unlike the variance and the standard deviation, the $\mathrm{CoV}$ also is suitable to compare data sets with largely different means [32]. To formally define the CoV, we first introduce the mean and the standard deviation.

The average of a set of scores $\left\{y_{1}, y_{2}, \ldots, y_{n}\right\}$ is called the mean of the observations or the mean of the population. It is obtained by dividing the sum of all observations by the number of the observations. The mean is usually denoted by $\mu$, and it is 
formally defined by

$$
\mu=\frac{1}{n} \sum_{i=1}^{n} y_{i} .
$$

The standard deviation is the square root of the average squared differences (deviations) of the scores from their mean value [31], i.e., it is the square root of the variance. The standard deviation is denoted $\sigma$, and it is computed as

$$
\sigma=\sqrt{\left(\frac{1}{n-1} \sum_{i=1}^{n}\left(y_{i}-\mu\right)^{2}\right)} .
$$

Formally, the $\mathrm{CoV}$ (denoted by $C$ ) of a set of observations $\left\{y_{1}, y_{2}, \ldots . . y_{n}\right\}$, i.e., scores or quantities, is defined to be the ratio

$$
C=\frac{\sigma}{\mu}
$$

where $\sigma$ and $\mu$ are the standard deviation and the mean, respectively.

A small CoV indicates that the scores tend to be close to each other (close to the mean of the population). On the other hand, a large CoV indicates that the scores are spread out over a wider range of values. Clearly, when the $\mathrm{CoV}$ is zero, it indicates that the scores have equal values, i.e., no variability.

As mentioned above, the $\mathrm{CoV}$ is a dimensionless quantity. Thus, it allows the comparison of distributions obtained with different units. In this thesis, we use the $\mathrm{CoV}$ of different geometric properties to measure the variability of the spatial pattern of points. These geometric properties have different units: meters $(\mathrm{m})$ and meters square $\left(\mathrm{m}^{2}\right)$. To demonstrate this dimensionless advantage, for example, consider the distances (in meters) between 10 users and their serving BS:

$\begin{array}{llllllllll}308 & 252 & 340 & 113 & 330 & 238 & 330 & 407 & 347 & 293\end{array}$


For this set of data, the mean distance is $\mu=295.8$ meters, the standard deviation is $\sigma=76.231$ meters, and the $\mathrm{CoV}$ is $C=0.2577$. Assume that this data is transformed to feet. Since there are approximately 3.25 feet in a meter, the set of data becomes:

$\begin{array}{llllllllll}1001 & 819 & 1105 & 367.25 & 1072.5 & 773.5 & 1072.5 & 1322.75 & 1127.75 & 952.25\end{array}$

Now, the mean distance is $\mu=961.35$ feet, the standard deviation is $\sigma=247.75$ feet. However, the $\mathrm{CoV}$ is $C=0.2577$; it is as before [31].

Another advantage is that the $\mathrm{CoV}$ can be used to compare the variability between two sets of data if their means widely differ [32]. It gives full information about the vriability of the sets of data and no other measures are required. On the other hand, the standard deviation have a meaning only in the context of the mean value of the data. For example, consider two sets of data generated using two Gaussian random variables, as shown in Figure 2.1. The mean, standard deviation, and CoV values of these two sets of data are $\left(\mu_{1}=10, \sigma_{1}=2.4, C_{1}=0.24\right)$ and $\left(\mu_{1}=100, \sigma_{1}=5\right.$, $\left.C_{2}=0.05\right)$, respectively.

The first set of data has a smaller standard deviation and larger $\mathrm{CoV}$ compared to the second set of data. Using the standard deviation and in the absence of the context of the mean value, it may appear that the second set of data has higher variability than the first set of data, but indeed the variability of the first set of data is higher $\left(C_{1}>C_{2}\right)$. Higher standard deviation value does not necessarily indicate higher variability, unless the compared sets of data have the same mean. 


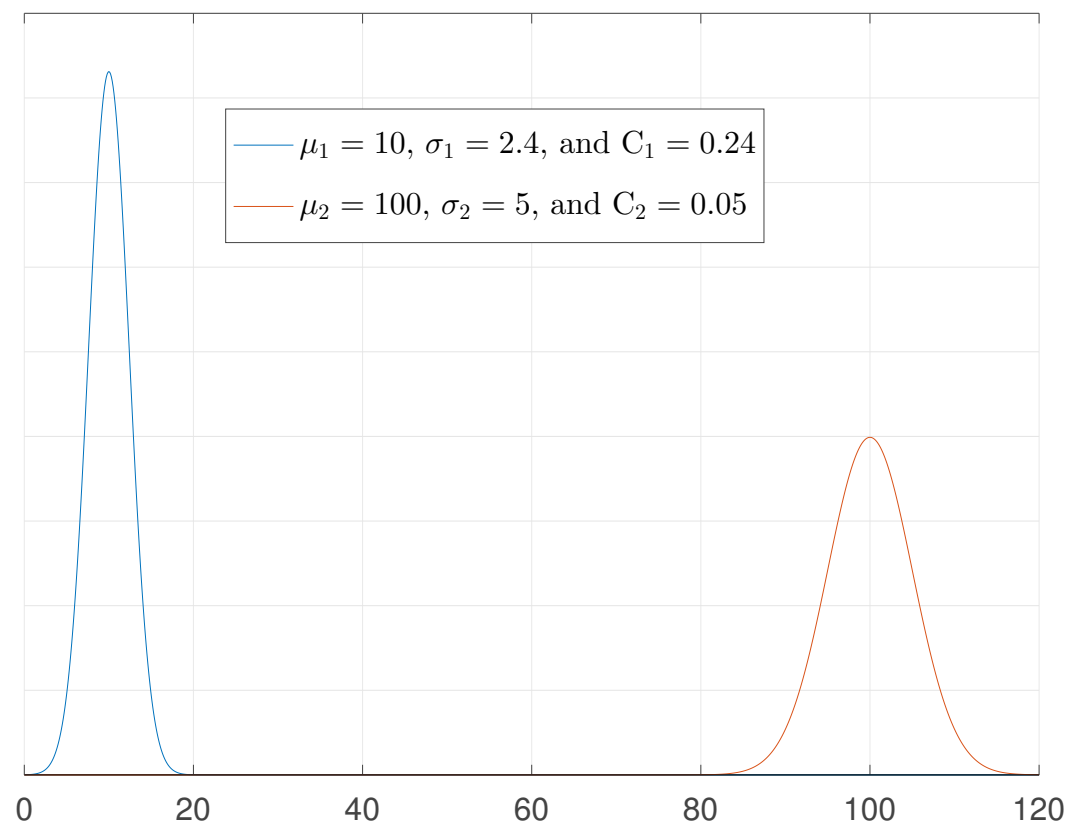

Figure 2.1: Probability density functions of two sets of data generated using two Gaussian random variables with mean, standard deviation, and $\mathrm{CoV}$ values $\left(\mu_{1}=10, \sigma_{1}=2.4, C_{1}=0.24\right)$ and $\left(\mu_{2}=100, \sigma_{2}=5, C_{2}=0.05\right)$, respectively.

\subsection{Geometry-Based (CoV-Based) Regularity Metrics}

The CoVs of three geometric properties have been introduced in [25-27] to measure the clustering of mobile user locations. We propose using these metrics to measure the amount of regularity of the BS locations. Each metric is normalized by a constant factor so that the $\mathrm{CoV}$ of the PPP is always equal 1.

\subsubsection{The CoV of the Distances to the Nearest Neighbour}

The distance to nearest neighbour have long been used for to quantify the spatial relationship in point patterns, e.g., in the ecology where the points represent a population of animals or plants [33] and more recent in sensor networks where the points represent the nodes [24]. 


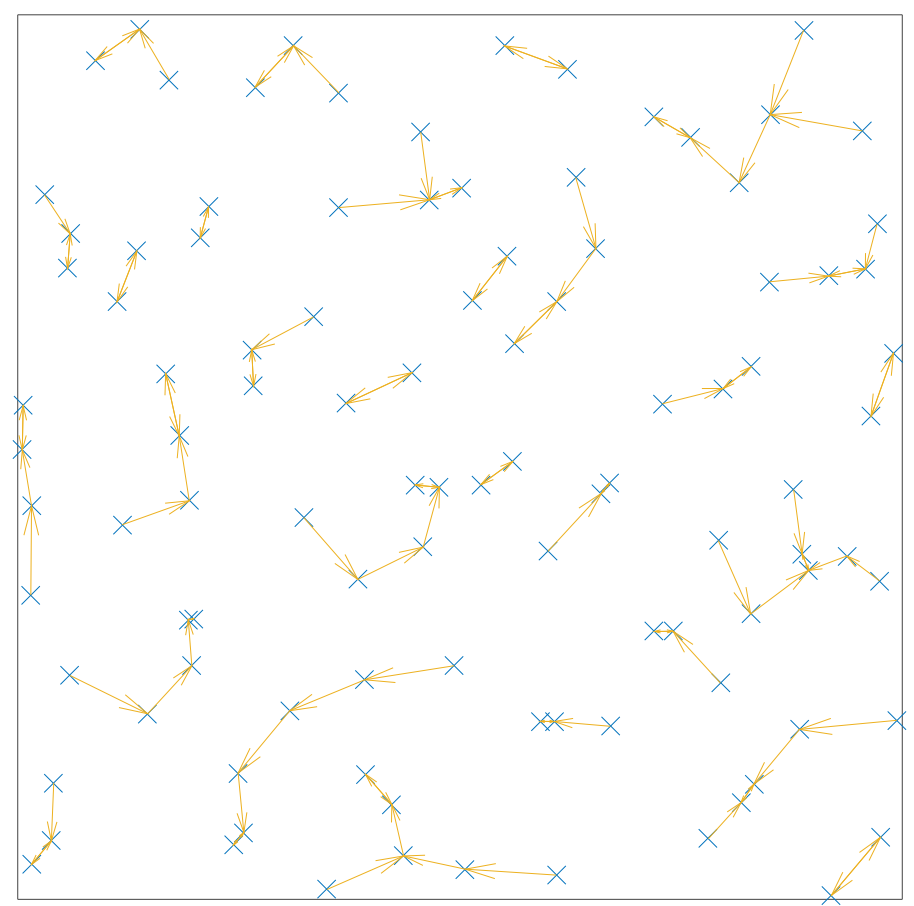

Figure 2.2: An arbitrary spatial point pattern (blue " $x$ " marks). The golden arrows indicate the distance from each point (at the arrow tail) to its nearest neighbour (at the arrowhead). Double-headed arrows means the points pair are the nearest neighbour of each other.

Taking the $\mathrm{CoV}$ of the distribution of the distance from every point to its nearest neighbour [34,35], the CoV-based metric is

$$
C_{\mathrm{N}}=\frac{1}{k_{\mathrm{N}}} \cdot \frac{\sigma_{\mathrm{N}}}{\mu_{\mathrm{N}}}, \quad k_{\mathrm{N}}=\sqrt{\frac{4-\pi}{\pi}} \cong 0.5227,
$$

where $\mu_{\mathrm{N}}$ is the mean and $\sigma_{\mathrm{N}}$ is the standard deviation of the nearest neighbour distances; and $k_{\mathrm{N}}$ is a normalization factor derived ${ }^{1}$ from [34]. Figure 2.2 depicts a set of points with their nearest neighbour.

\footnotetext{
${ }^{1}$ In [25], the value for $k_{\mathrm{N}}$ is erroneously given as 0.653 .
} 


\subsubsection{The CoV of the Areas of Voronoi Tessellation Cells}

The Voronoi cell $C_{i}$ of a point $x_{i}$ belongs to a spatial point pattern $\mathrm{X}=$ $\left\{x_{1}, x_{2}, \ldots, x_{\mathrm{n}(\mathrm{X})}\right\} \subset \mathbb{R}^{d}$, where $\mathrm{n}(\mathrm{X})=|\mathrm{X}|$ is the number of the points, consists of all locations that are closer to the point $x_{i}$ than any other point $x_{j} \in \mathrm{X}$. This can be expressed formally $[5,6,36]$ as

$$
C_{i}=\left\{y \in \mathbb{R}^{d}:\left\|y-x_{i}\right\| \leq\left\|y-x_{j}\right\|, \quad \forall i \neq j\right\} .
$$

The Voronoi tessellation is a subdivision of the space of the spatial point pattern $\mathrm{X}$ into the Voronoi cells $[5,6]$. Figure 2.3 demonstrates patterns of points with their Voronoi tessellation (blue solid lines).

Considering the areas of the cells of the Voronoi tessellation of a set of points, the $\mathrm{CoV}$ of the distribution of the areas of the Voronoi cells is another CoV-based metric, which is then

$$
C_{\mathrm{V}}=\frac{1}{k_{\mathrm{V}}} \cdot \frac{\sigma_{\mathrm{V}}}{\mu_{\mathrm{V}}}, \quad k_{\mathrm{V}} \cong 0.529,
$$

where $\mu_{\mathrm{V}}$ is the mean and $\sigma_{\mathrm{V}}$ is the standard deviation of the Voronoi cell areas, and $k_{\mathrm{V}}$ is a normalization factor [25].

\subsubsection{The CoV of the Lengths of Delaunay Triangulation Edges}

The Delaunay triangulation of a set of points in a plane can be constructed by connecting the points that have contiguous Voronoi cells [5]. The Delaunay triangulation (golden dashed lines) of different spatial patterns is illustrated in Figure 2.3. 
Taking the edges of the Delaunay triangulation [5] of a set of points, the CoVbased metric is

$$
C_{\mathrm{D}}=\frac{1}{k_{\mathrm{D}}} \cdot \frac{\sigma_{\mathrm{D}}}{\mu_{\mathrm{D}}}, \quad k_{\mathrm{D}} \cong 0.492
$$

where $\mu_{\mathrm{D}}$ is the mean and $\sigma_{\mathrm{D}}$ is the standard deviation of the Delaunay edge lengths, and $k_{\mathrm{D}}$ is a normalization factor [25].

(Note that in order to eliminate the edge effect, Delaunay edges between points on the border of the considered region should not be taken into consideration when calculating the $C_{\mathrm{D}}$. Similarly, the Voronoi cell areas and the nearest neighbour distances of the border points should not be taken into consideration when calculating the $C_{\mathrm{V}}$ and $C_{\mathrm{N}}$, respectively.)

Figure 2.3 shows realizations of four different network layouts with different amounts of regularity as measured using the $C_{\mathrm{D}}$ metric. The perfect hexagonal layout (Figure 2.3(a)) has $C_{\mathrm{D}}=0$ (completely regular). At the other end of the RPPs scale, the PPP (Figure 2.3(d)) has $C_{\mathrm{D}}=1$ (a completely random layout). In general, the CoV-based metrics take the value of 0 for a triangular lattice and 1 for a PPP. Values between 0 and 1 are found for RPPs, as will be seen in the next chapters, while values above 1 are found for point processes with clustering [25]. Practical models for the BS locations should have a $\mathrm{CoV}$ between 0 and 1. As the BSs spread out, the regularity increases. With the aid of one of the geometry-based metrics, we can distinguish different spatial patterns in a quantitative manner.

For a stationary spatial pattern, the geometry-based metrics are density independent, and their values do not change under a dilation transformation, i.e., enlargement or reduction of a spatial pattern by a given factor. That is to say:

- the spatial pattern properties are preserved under dilation, where a resized spatial pattern with a particular scaling factor has the same amount of regularity 


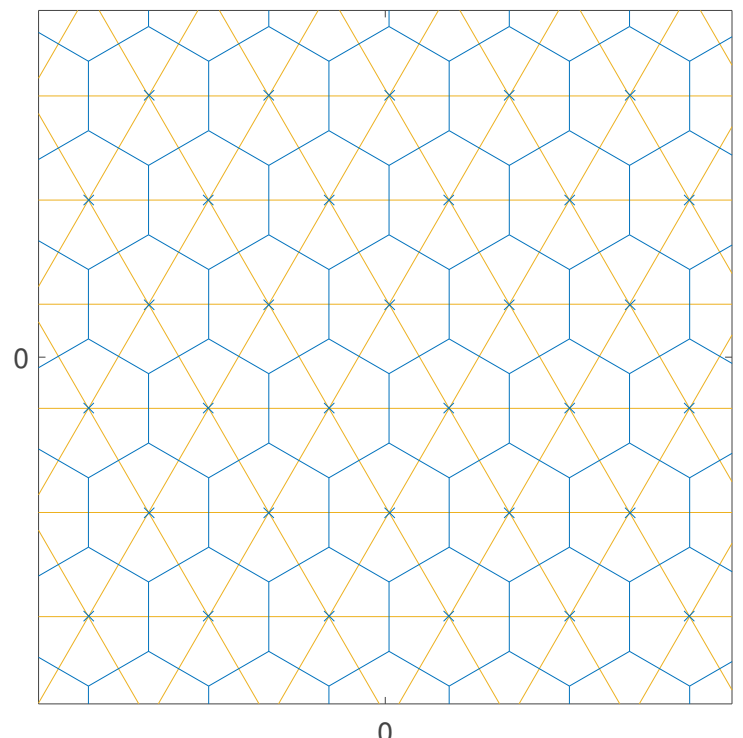

(a) $C_{\mathrm{D}}=0$

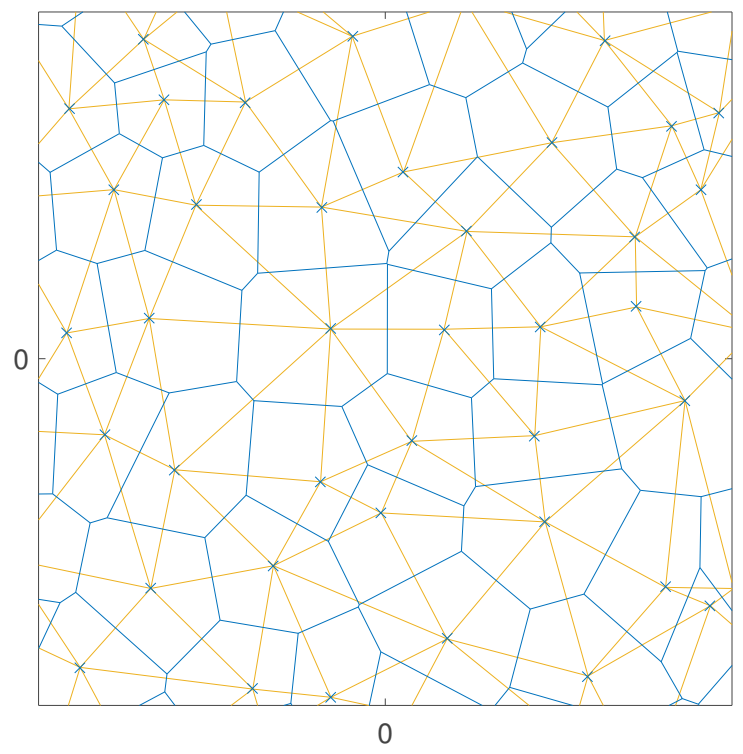

(c) $C_{\mathrm{D}}=0.6$

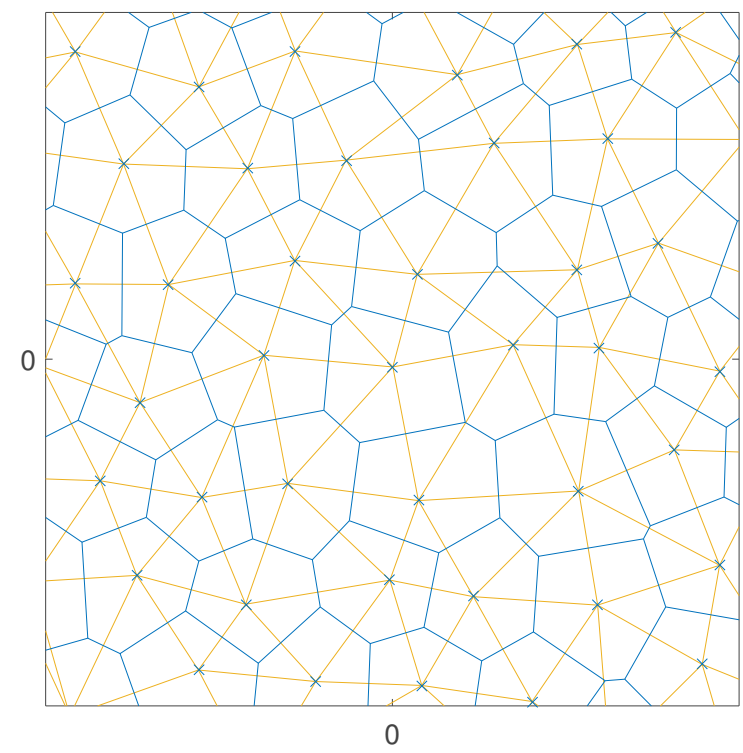

(b) $C_{\mathrm{D}}=0.4$

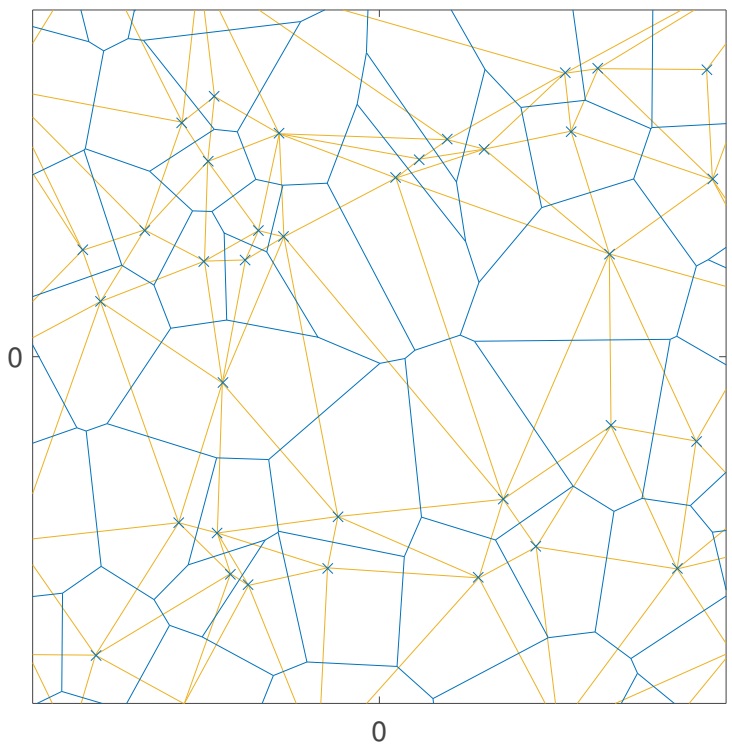

(d) $C_{\mathrm{D}}=1(\mathrm{PPP})$

Figure 2.3: Delaunay triangulation (golden dashed lines) and Voronoi tessellation (blue solid lines) of a spatial pattern (blue plus marks) with different amounts of regularity as quantified using $C_{\mathrm{D}}$. 
as the original spatial pattern.

- If we select a fixed size (fixed area or borders) polygonal window that is representing a sub-region within a whole region and then resize the whole spatial pattern, the density of the points inside the polygon changes proportionally with the scaling factor, but the amount of regularity as measured using the geometrybased metrics remains constant. A tiny variation may happen because of the edge effect.

- Two spatial patterns which are generated using a particular point process with the same normalized parameters but with different densities have on the average equal amount of regularity.

In short, the metrics $C_{\mathrm{V}}, C_{\mathrm{D}}$, and $C_{\mathrm{N}}$ are unit-less quantities and are invariant under scaling of the measured point process, and can thus be adjusted independently of its density $\lambda$.

The three geometric properties are also meaningful in the context of BS locations: the Voronoi tessellation represents the cell area associated with each BS under the assumption the users always connect to the nearest BS [37], while the Delaunay triangulation connects each BS to its strongest interfering BSs, and the nearest neighbour characterizes the dominating interfering BS and has been of interest in measuring the regularity of RPPs [24].

\subsection{Concluding Remarks}

We proposed three different CoV-based metrics to measure the amount of regularity of spatial point processes used in cellular networks to model the locations of BSs. These metrics have many theoretical and practical application in cellular networks 
as shown in this thesis. Indeed, these metrics are also applicable for measuring the spatial relationship to all stationary point processes, in any field of study. 


\section{Chapter 3}

\section{Hard-Core Point Processes for Modelling the Locations of Base Stations}

"The beginning of knowledge is the discovery of something we do not understand."

-Frank Herbert

\subsection{Introduction}

In this chapter ${ }^{1}$, we examine three hard-core point processes for modelling the locations of the BSs regarding the spatial regularity range and the point generation efficiency. We use the CoV-based metrics, introduced in Chapter 2, for measuring the regularity. These hard-core point processes are already proposed in wireless literature to model BS locations [5,22,30,38,39], which are Matérn hard-core processes of type I (MHC-I), Matérn hard-core processes and type II (MHC-II), and simple sequential inhibition (SSI) process [5]. Other RPPs used in the literature include determinantal point process models $[16,17]$, and the family of Gibbs point processes $[13,15]$. RPPs are also useful for modelling heterogeneous networks (HetNets) [14] and wireless sensor networks [24].

\footnotetext{
${ }^{1}$ This chapter has been published in the IEEE Wireless Communication Letters [23].
} 


\subsubsection{Contributions}

We evaluate three geometry-based metrics, introduced in Chapter 2, for measuring the regularity of three common hard-core point processes in wireless literature. These point processes have only two tuning parameters: the density and the hard-core distance. This chapter's contributions are as follows:

1. We evaluate the use of three geometry-based metrics for quantifying the amount of regularity,

2. we show that the geometry-based metrics are capable of measuring the amount of regularity of RPPs and that the $\mathrm{CoV}$ of the nearest neighbour distance is the most sensitive metric among them, and

3. we compare the hard-core point processes as models for BS locations in terms of the achievable range of regularity.

The rest of this chapter is organized as follows: In Section 3.2, three hard-core point processes are introduced. In Section 3.3, the geometry-based metrics are evaluated for quantifying the regularity of simulated realizations of the hard-core point processes. Finally, we compare one geometry-based metric with function-based metrics in Section 3.4, before drawing conclusions in Section 3.5.

\subsection{Hard-Core Point Processes}

A spatial point process $\mathrm{X}$ on a domain $W$ produces random realizations of points

$\left(x_{1}, x_{2},\right)$ in the 2 -dimensional plane $\left(x_{i} \in \mathbb{R}^{2}\right)$. For a single realization, the number of points falling in a set $B \subset W \subseteq \mathbb{R}^{2}$ is is a random variable denoted by $\mathrm{n}(B)$, or 


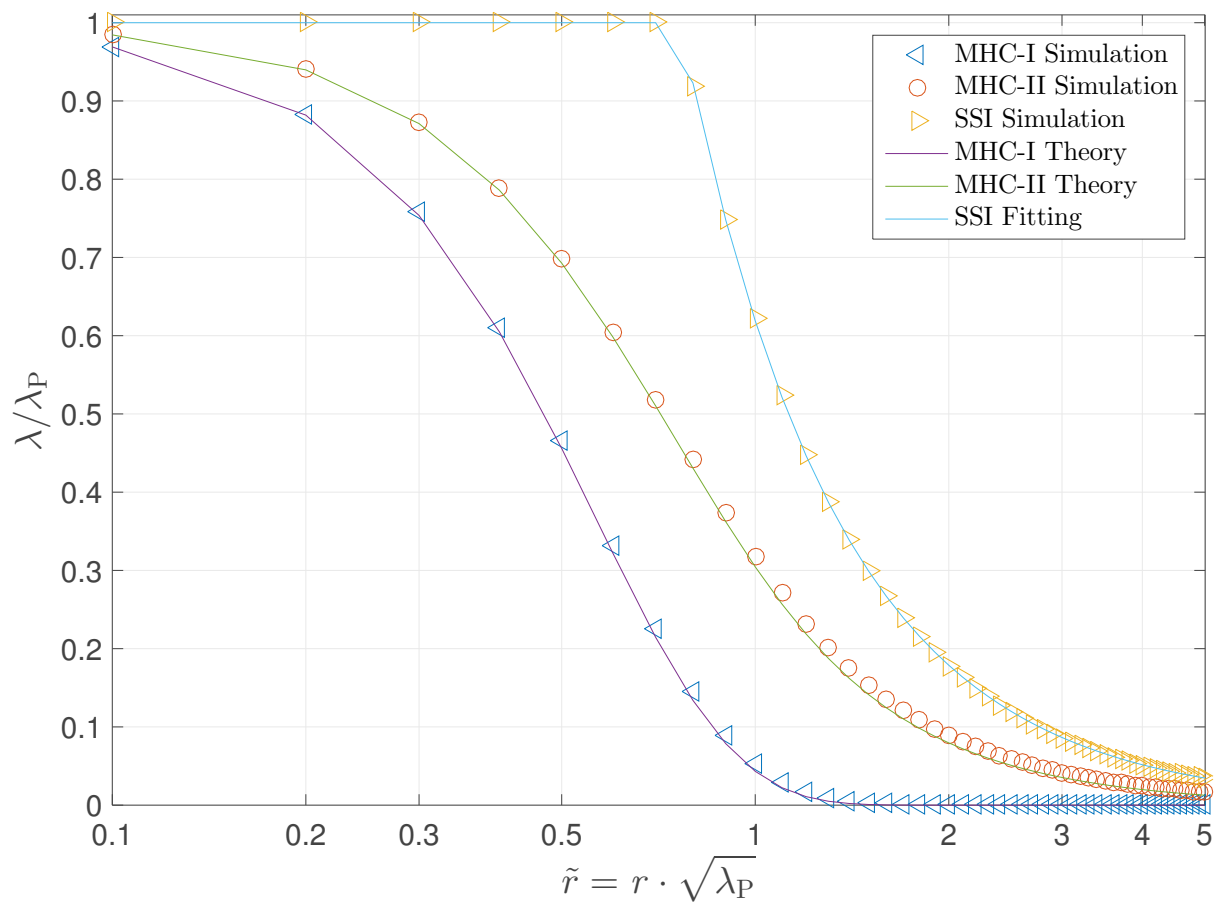

Figure 3.1: The density ratio of MHC-I, MHC-II, and SSI as a function of $\tilde{r}$.

sometimes denoted by $\mathrm{n}(\mathrm{X})$. Different realizations have on average the same number of points.

A hard-core point processes $\mathrm{X}$ is a RPP where two points are strictly prohibited from being closer than a predefined hard-core distance $r>0$ apart $[5,22,37]$. Formally, a hard-core point processes can be expressed as

$$
\mathrm{X}=\left\{x_{i} \in \mathbb{R}^{2}:\left\|x_{i}-x_{j}\right\| \geq r ; i \neq j ; i, j \in \mathbb{Z}_{+}\right\}
$$

where $\|\cdot\|$ is the Euclidean distance.

In this section, we describe three hard-core point processes. In general, generation of these three hard-core point processes begins with generating a PPP, and then removing points that violate the hard-core condition. Different ways of removing points (removing points is called thinning) lead to different RPPs with different densities. 


\subsubsection{Matérn Hard-Core Process of Type I (MHC-I)}

The MHC-I is generated as follows: From a PPP $\Phi$ with density $\lambda_{\mathrm{P}}$, simultaneously remove all points that are closer than $r$ from each other. The MHC-I can be expressed as

$$
X_{\mathrm{I}}=\left\{x_{i} \in \Phi:\left\|x_{i}-x_{j}\right\| \geq r, \forall x_{j} \in \Phi ; i \neq j ; i, j \in \mathbb{Z}_{+}\right\}
$$

The density of the MHC-I is [5]

$$
\lambda=\lambda_{\mathrm{P}} e^{-\lambda_{\mathrm{P}} \pi r^{2}}
$$

and its normalization by $\lambda_{\mathrm{P}}$ is

$$
\frac{\lambda}{\lambda_{\mathrm{P}}}=e^{-\pi \tilde{r}^{2}}
$$

where $\tilde{r}=r \sqrt{\lambda_{\mathrm{P}}}$ is the normalized hard-core distance.

\subsubsection{Matérn Hard-Core Process of Type II (MHC-II)}

The MHC-II is generated by following three steps: First, generate a PPP $\Phi$ with density $\lambda_{\mathrm{P}}$. Second, associate a mark $U_{i}$ which is an independent uniform random variable on $[0,1]$ to each point $x_{i} \in \Phi$. Then, simultaneously remove all points that have higher marks than their neighbours within a distance $r$. The MHC-II can be written as

$$
X_{\mathrm{II}}=\left\{x_{i} \in \Phi: U_{i}<U_{j}, \forall x_{j} \in \Phi \cap B\left(x_{i}, r\right) \backslash\left\{x_{i}\right\} ; i \neq j ; i, j \in \mathbb{Z}_{+}\right\}
$$


where $B\left(x_{i}, r\right)$ is a ball with centre $x_{i}$ and radius $r$. The density of the MHC-II is [5]

$$
\lambda=\frac{1-e^{-\lambda_{\mathrm{P}} \pi r^{2}}}{\pi r^{2}}
$$

and its normalized density is

$$
\frac{\lambda}{\lambda_{\mathrm{P}}}=\frac{1-e^{-\lambda_{\mathrm{P}} \pi r^{2}}}{-\lambda_{\mathrm{P}} \pi r^{2}}=\frac{1-e^{-\pi \tilde{r}^{2}}}{\pi \tilde{r}^{2}} .
$$

\subsubsection{Simple Sequential Inhibition (SSI)}

Given the required density of points $\lambda_{\mathrm{P}}$ in a domain, candidate points are generated sequentially using a PPP. Points are discarded if they are within a distance $r$ from any previously accepted point. The process terminates when the required den-

sity is attained or when adding more point becomes impossible [5,40]. The density of the accepted points is $\lambda$. We are not aware of any closed-form expression for the SSI density. Therefore, we conduct a curve-fitting, and we find a good fit for our results:

$$
\frac{\lambda}{\lambda_{\mathrm{P}}}=\min \left\{1, \quad 0.61 \tilde{r}^{-1.79}\right\}
$$

The density ratios as a function of $\tilde{r}$ for MHC-I, MHC-II, and SSI, given by (1)-(3), are shown in Figure 3.1. 


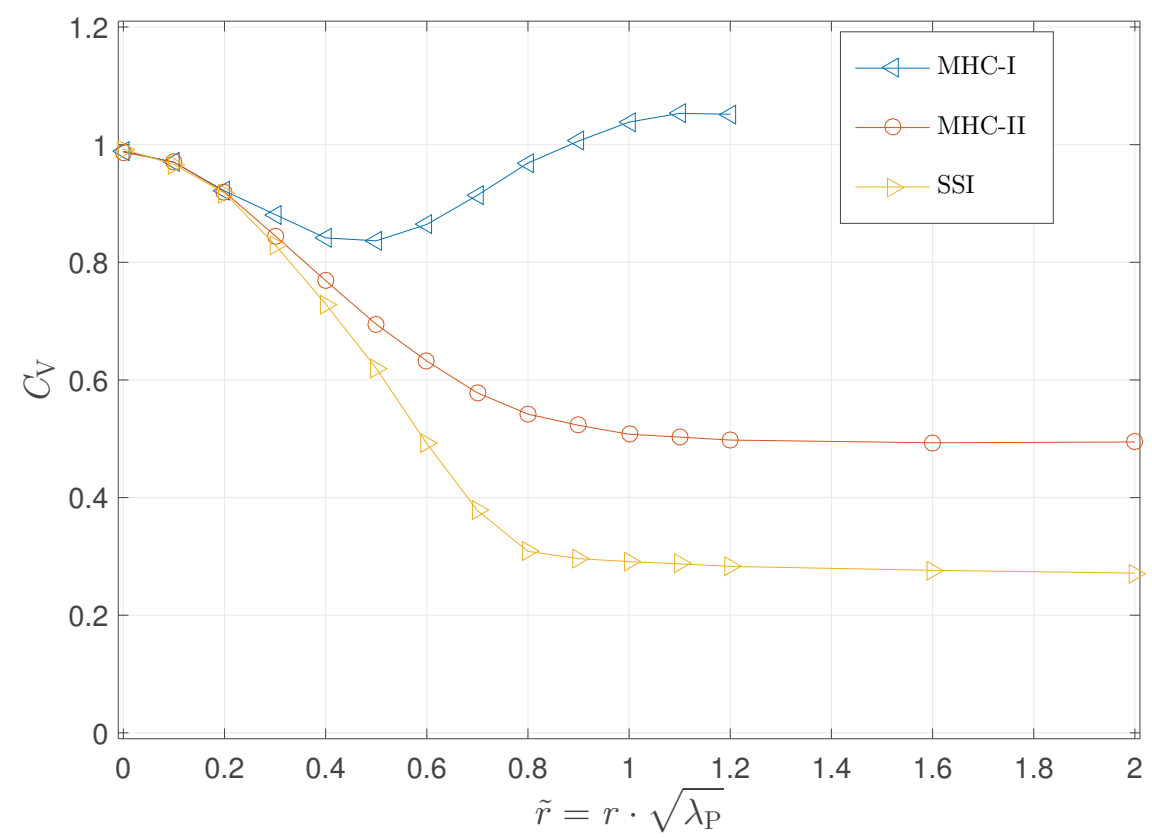

Figure 3.2: The CoV of the areas of the Voronoi tessellation cells as a function of the normalized hard-core distance for hard-core point processes.

\subsection{Evaluation of CoV-based Metrics for Hard- Core Point Processes}

We generate spatial patterns of BS locations using the point processes defined in Section II and measure their amount of regularity using the metrics defined in Chapter 2.

The density $\lambda$ is fixed to be 100 points in a $1 \mathrm{~km}^{2}$ square domain. $\tilde{r}$ is swept over a wide range to change the regularity of the RPP, which is captured using the CoV-based metrics presented in Chapter 2. For each RPP and metric combination, a Monte-Carlo simulation is performed with 1000 realizations. The ensemble mean of the CoV-based metrics of the resulting points as a function of $\tilde{r}$ is shown in Figures 3.2, 3.3 , and 3.4 . 


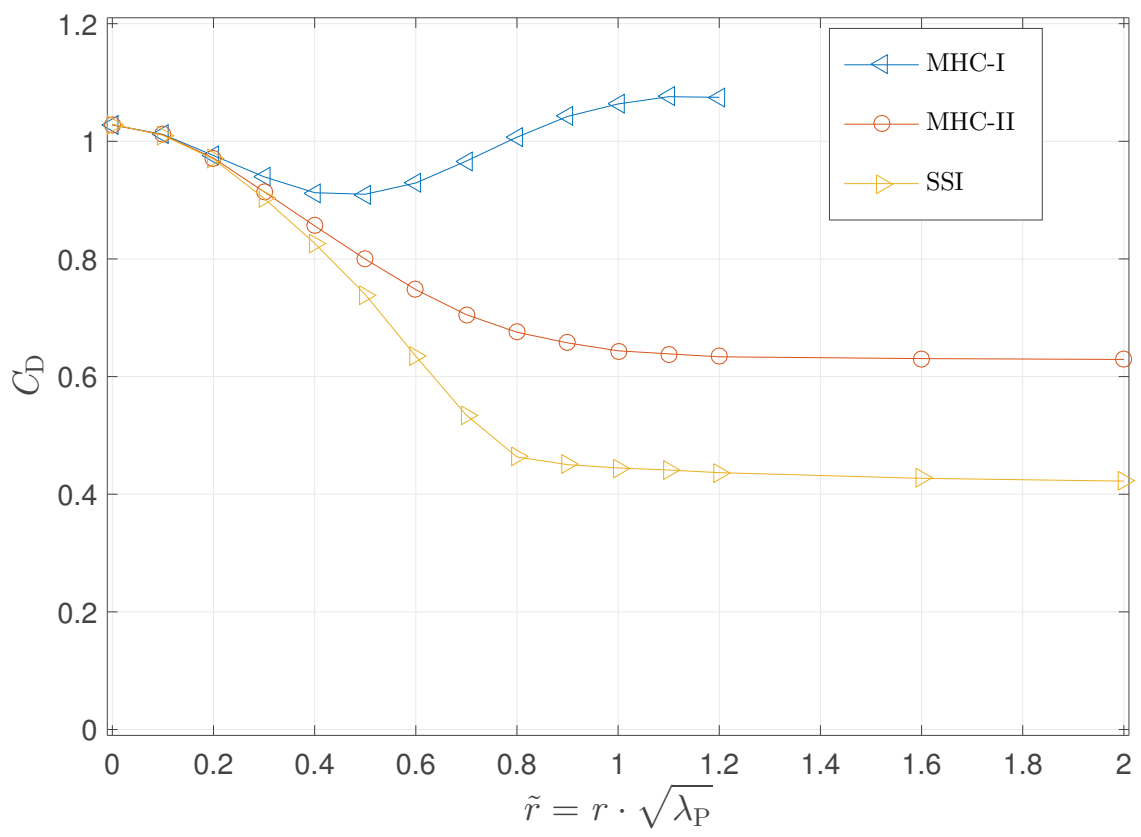

Figure 3.3: The CoV of the lengths of the Delaunay triangulation edges as a function of the normalized hard-core distance for hard-core point processes.

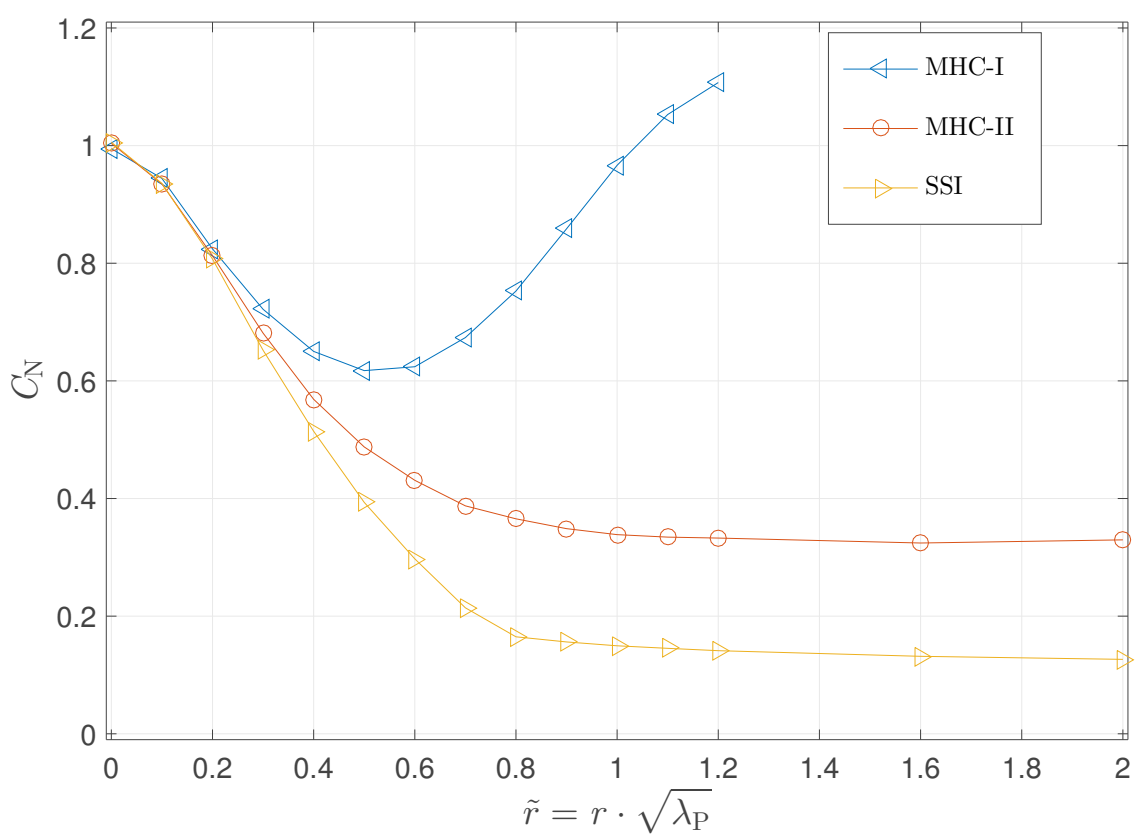

Figure 3.4: The $\mathrm{CoV}$ of the distance to the nearest neighbour as a function of the normalized hard-core distance for hard-core point processes. 
Table 3.1: CoV-based metrics floor for hard-core point processes

\begin{tabular}{|c|c|c|c|c|}
\cline { 3 - 5 } \multicolumn{1}{c|}{} & $C_{\mathrm{V}}$ & $C_{\mathrm{D}}$ & $C_{\mathrm{N}}$ \\
\hline \multirow{3}{*}{ CoV floor } & MHC-I & 0.84 & 0.91 & 0.62 \\
\cline { 2 - 5 } & MHC-II & 0.49 & 0.63 & 0.33 \\
\cline { 2 - 5 } & SSI & 0.27 & 0.42 & 0.13 \\
\hline
\end{tabular}

We observe that the SSI process has the widest CoV ranges $^{2}$ and achieves the highest density ratio, making it the most attractive hard-core RPP. Conversely, the MHC-I process is the least desirable among the investigated RPPs. It has the lowest density ratio, making it inefficient in generating a given number of points, and its $\mathrm{CoV}$ values fluctuate in a narrow range around 1 . We interpret this behaviour as being caused by the nature of the MHC-I process itself: Removing all points that violate the hard-core condition creates large holes in the generated pattern when the hard-core distance is large, causing some of the remaining points to cluster, relatively speaking. Since cluster processes were shown [25] to have CoVs greater than 1, this clustering increases the $\mathrm{CoV}$ value of MHC-I.

Our results also show that (i) the amount of regularity of hard-core point processes is tunable and can be quantified using CoV-based metrics and (ii) the useful tuning range of these RPPs is $\tilde{r}<1$, i.e., where the CoVs are sensitive to changes in $\tilde{r}$.

The ranges of the CoV-based metrics are summarized in Table I. The $C_{\mathrm{N}}$ metric provides the widest value range, making it the most sensitive to changes in the amount of regularity of the RPP.

\footnotetext{
${ }^{2}$ Indeed, in [30], SSI is observed to be more regular than MHC-II.
} 


\subsection{Comparison of $C_{\mathrm{N}}$ with two Function-Based Metrics}

In this section, we choose $C_{\mathrm{N}}$ to examine its relationship to two function-based metrics: the Ripley's K-function and the coverage probability — a network performance metric.

\subsubsection{Ripley's K-Function}

Ripley's K-function ${ }^{3} K(r)$ is defined as the ratio of the mean number of extra points within distance $r$ from a typical point (not included in the counting) to the density of the spatial pattern $[22,24]$. It can characterize the regularity or clustering of a point process. The L-function, $L(r)=\sqrt{(K(r) / \pi)}$, is a normalized form of $K(r)$. While $L(r)=r$ for a PPP, a spatial pattern with $L(r)<r$ is repulsive.

As shown in Figure 3.5, a hard-core RPPs with the same $C_{\mathrm{N}}$ value have similar L-function and hard-core distance $r$, apart from the MHC-I in the second regime (in which the $\mathrm{CoV}$ value increases with $\tilde{r}$ ). This indicates that matching RPPs using Ripley's functions is not always possible as the curves can have very different shapes.

\subsubsection{Coverage Probability}

The coverage probability $P(\gamma)$ is the probability that a typical user achieves a signal-to-interference ratio (SIR) higher than a given SIR threshold $\gamma$. We compare the downlink coverage probability where the BSs are deployed according to hardcore point processes introduced in Section 3.2 with different amounts of regularity

\footnotetext{
${ }^{3}$ The Ripley's K-functions of MHC-I and MHC-II are known in a complicated integral form [22].
} 


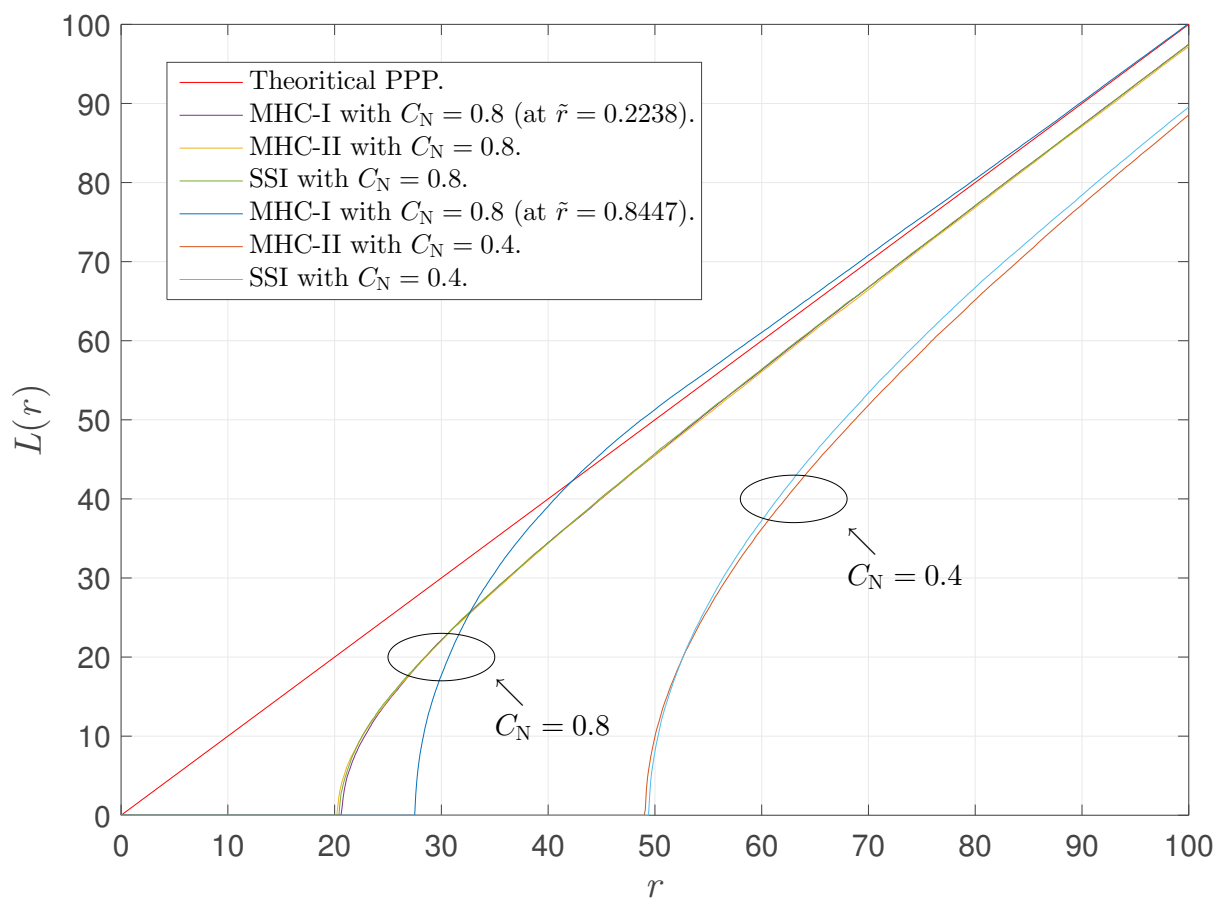

Figure 3.5: Normalized Ripley's K-function as a function of the hard-core distance $r$ (meters) for different RPPs and $C_{\mathrm{N}}$ values.

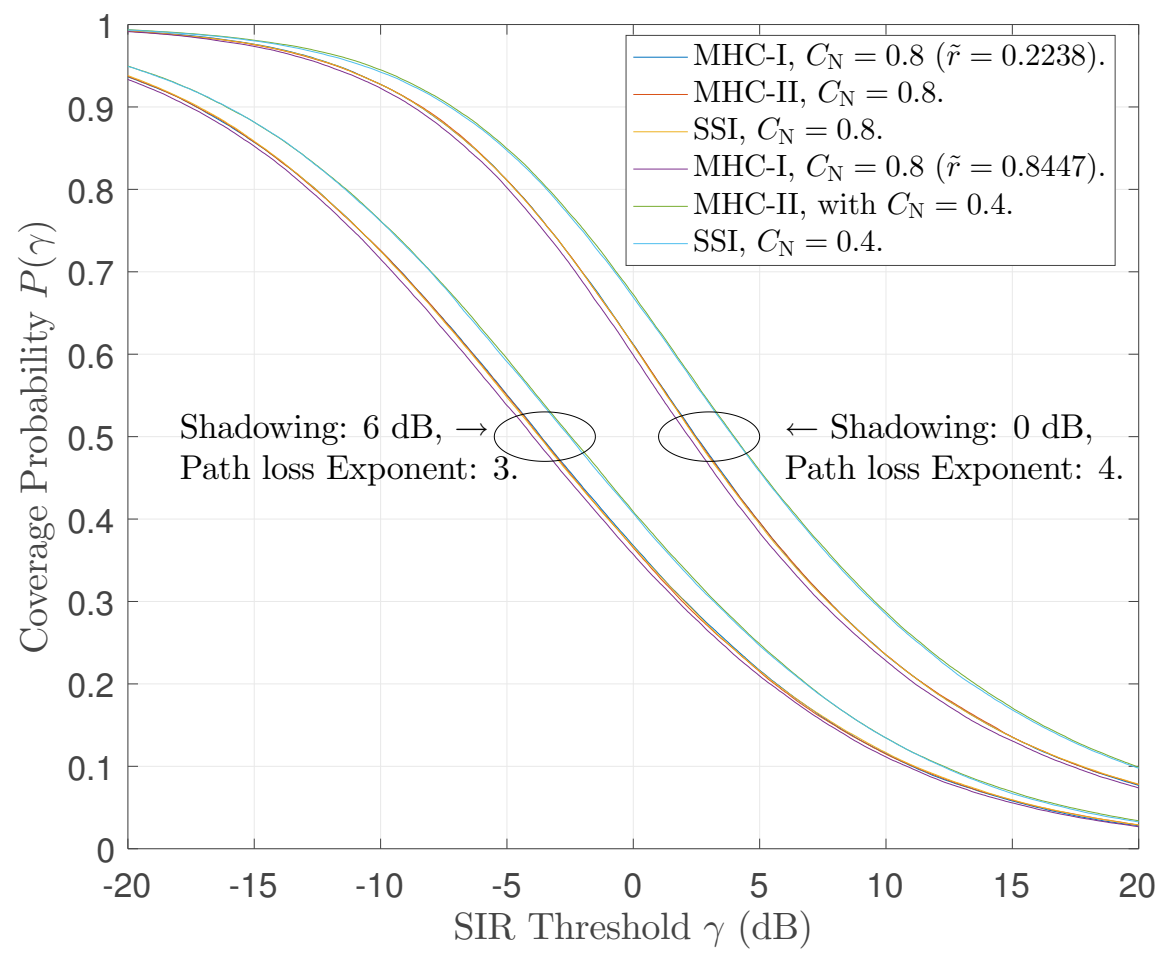

Figure 3.6: The coverage probability for different hard-core models with constant density for different $C_{\mathrm{N}}$ values and channel environments. 
as measured using $C_{\mathrm{N}}$. The following assumptions are used to evaluate the coverage probability: (i) The average density is $100 \mathrm{BSs}$ in $1 \mathrm{~km}^{2}$, (ii) all BSs transmit the same power, (iii) mobile users are uniformly distributed over the entire domain and each of them is associated to its nearest BS, (iv) the frequency reuse factor is 1 , (v) all channels have Rayleigh fading with mean 1, and (vi) the thermal noise is ignored. We also assumed two channel models: one with a path loss exponent of $\alpha=3$ and 6 dB lognormal shadowing, and one with $\alpha=4$ and no shadowing.

Figure 3.6 shows that different hard-core RPPs with the same $C_{\mathrm{N}}$ in different channel environments behave alike regarding coverage probability. This is true even for MHC-I in the second regime.

$C_{\mathrm{N}}$ is an additional factor that affects the network performance. A spatial pattern with a low $C_{\mathrm{N}}$ value has a better performance than one with a high $C_{\mathrm{N}}$ value. BSs deployed according to the same density and $C_{\mathrm{N}}$ have very similar SIR performance, regardless of the chosen hard-core point process.

\subsection{Concluding Remarks}

- We found that $C_{\mathrm{N}}$ is the most sensitive to the regularity of RPPs (i.e., most sensitive to the change of the internal parameters of the RPPs).

- We observe that different BS location models with the same density and $C_{\mathrm{N}}$ value have very similar SIR performance. In Chapter 4 and Chapter 5, we conduct more investigations to find the best regularity metric for measuring the regularity and for capturing the network performance.

- Our results also show that the MHC-I process is undesirable for modelling points with regularity, whereas both the MHC-II and SSI processes are useful when 
their normalized hard-core distance is less than 1, SSI being the best in terms of the range of regularity and density ratio.

- Given real deployments of BS locations, an interesting extension is to investigate whether the CoV-based metrics could work as a tool for fitting them to RPP models. Chapter 5 considers this research direction.

- Another extension could include investigating other RPPs. In Chapter 4, we examine the PTL to model the spatial deployment of the BSs, and, in Chapter 5, we examine Strauss point process. 


\section{Chapter 4}

\section{Perturbed Triangular Lattice for Modelling the Locations of Base Stations}

"Essentially, all models are wrong, but some are useful."

-George Box

\subsection{Introduction}

In Chapter 3, we evaluated three hard-core point processes for modeling BS locations: the Matérn hard-core processes of type I and type II [5,22], and the simple sequential inhibition (SSI) process [5]. In Chapter 3, we also used three CoV-based metrics (introduced in Chapter 2) to quantify the regularity of these hard-core point processes. (As mentioned before, these metrics were originally proposed in [25-27] for measuring the attraction in clustered point processes.) All these hard-core point processes are based on generating independent points, and then removing certain points based on some formula involving their mutual distances and sequential order. Therefore, one important limitation of these RPPs is their inability to provide very high regularity $[13,23]$, as it is not possible to obtain a TL from thinning a PPP. It is also often necessary to generate a large number of points (and evaluate the distance between each pair), in order to obtain a smaller number of points at the output, 
making these methods computationally costly.

In order to overcome these limitations, in this chapter, ${ }^{1}$ we advocate the use of the perturbed triangular lattice (PTL), which can be tuned over the whole range between the deterministic TL and the PPP [15,42], for modelling the locations of the BSs. The PTL can be scaled with different densities for different simulation scenarios without changing the regularity of the deployment. Recently, the uniform PTL is found to be tractable in [42]. The PTL is also a simple model to simulate; as a consequence, it is practical and widely used in the industry [15].

\subsubsection{Motivation}

One of the challenges in BS location modelling is how to adjust the internal parameters of different models to produce spatial patterns with the same amount of regularity, or to check whether two given spatial patterns are similar or not. The first step to overcome this challenge is to find good metrics for the regularity of a spatial pattern in order to precisely define where it lies between the TL and the PPP. The second step, which is the focus of this chapter, is to use these metrics as an intermediate stage to map different models to each other.

\subsubsection{Contribution}

The main contribution of this chapter is proposing a novel approach for mapping between different RPPs using CoV-based metrics. Specifically, we find a simple and accurate formula for mapping between the uniform PTL and the Gaussian PTL. We show that the CoV-based metrics are suitable for quantifying the regularity of PTL lattice models. Similar to Chapter 3, where we found that different hard-core

\footnotetext{
${ }^{1}$ This chapter has been published in the IEEE 84th Vehicular Technology Conference (VTC2016Fall) [41].
} 
models with the same CoV-based metric value and density have very similar network performance, here we show that this is true for different PTLs as well.

The rest of this chapter is organized as follows: In Section 4.2, perturbed triangular lattice models are introduced. In Section 4.3, we measured the regularity of the perturbed triangular lattices using the $\mathrm{CoV}$-based metrics to quantify regularity. We proposed new a approach using the CoV-based metrics for mapping between two PTL models in Section 4.4. Finally, we draw the conclusions in Section 4.5.

\subsection{Perturbed Triangular Lattices Models for Base Stations Placement}

In the cellular network context, the triangular lattice (TL) is often used to model the layout of BSs. The Voronoi tessellation of the TL produces hexagonal cells. (Erroneously, it is sometimes also called the hexagonal lattice. Indeed, the hexagonal lattice is the dual of the Triangular Lattice [43].) We use the triangular lattice term throughout this thesis. The PTL is a result of independent random displacement of the points from their original locations. In this section, we present two kinds of the perturbed triangular lattices: Gaussian PTL and uniform PTL.

Triangular Lattice Beginning with a unit square lattice with integer coordinates $(a, b) \in \mathbb{Z}^{2}$, a triangular lattice can be produced by transforming the points using the generator matrix $G=\left[\begin{array}{cc}1 & 0 \\ \frac{1}{2} & \frac{\sqrt{3}}{2}\end{array}\right]$ and scaling the coordinates by a factor $\eta$, which is the distance between any two nearest neighbour points. The TL has points with coordinates

$$
\left\{\left(\eta a+\frac{1}{2} \eta b, \frac{\sqrt{3}}{2} \eta b\right),(a, b) \in \mathbb{Z}^{2}\right\}
$$


The TL has density $\lambda=\frac{1}{\operatorname{det}(G)} \eta^{-2}=\frac{2}{\sqrt{3}} \eta^{-2}$.

Stationary Triangular Lattice Translation of all the lattice points by a common vector $\left(s_{\mathrm{x}}, s_{\mathrm{y}}\right)$ uniformly distributed over the Voronoi cell of the origin point $(0,0)$ results in a stationary point process [13]. For the triangular lattice, the Voronoi cell is a regular hexagon ${ }^{2}$ with two vertical sides, ${ }^{3}$ centred at the origin with side length $\eta / \sqrt{3}$. The stationary triangular lattice has coordinates

$$
\left\{\left(\eta a+\frac{1}{2} \eta b+s_{\mathrm{x}}, \frac{\sqrt{3}}{2} \eta b+s_{\mathrm{y}}\right),(a, b) \in \mathbb{Z}^{2}\right\},
$$

where $\left(s_{\mathrm{x}}, s_{\mathrm{y}}\right)$ is uniformly distributed over the aforementioned hexagon. All PTL models are assumed to be stationary in this thesis.

Perturbed Triangular Lattices For any perturbed lattice, each point is independently displaced by random vector $\left(x_{(a, b)}, y_{(a, b)}\right)$ [5]. The PTL has coordinates

$$
\left\{\left(\eta a+\frac{1}{2} \eta b+s_{\mathrm{x}}+x_{(a, b)}, \frac{\sqrt{3}}{2} \eta b+s_{\mathrm{y}}+y_{(a, b)}\right),(a, b) \in \mathbb{Z}^{2}\right\},
$$

where $\left\{\left(x_{(a, b)}, y_{(a, b)}\right)\right\}$ is a set of independent and identically distributed vectors. The distribution from which the vectors are drawn characterizes the type of PTL.

In this thesis, we study two kinds of perturbation: Gaussian and uniform. The uniform PTL is one where the displacement vectors are uniformly distributed over a disc of radius $R$, which controls the amount of perturbation and can be normalized as $\tilde{R}=R \eta^{-1}$ to be independent of the density. Similarly, the Gaussian PTL is one where the displacement coordinates $x_{(a, b)}$ and $y_{(a, b)}$ are each taken independently from

\footnotetext{
${ }^{2}$ It is simpler to generate a stationary square lattice, where the Voronoi cell is a square, and then transform it into a stationary triangular lattice using the generator matrix $G$.

${ }^{3}$ If a random rotation is introduced to generate an isotropic triangular lattice, the hexagon no longer has a two vertical sides. They would have a tilted position according to the rotation angle.
} 
a Gaussian distribution with mean 0 and standard deviation $\sigma$, which controls the amount of perturbation, and can be normalized as $\tilde{\sigma}=\sigma \eta^{-1}$ to be independent of the density.

An interesting observation about the uniform perturbation on a disc is that the model can work either as a soft-core or as a hard-core model depending on the perturbation regime. It is a hard-core model in the regime $\tilde{R}<0.5$ (the hard-core distance is then $h=(1-2 \tilde{R}) \eta)$ and is a soft-core model after that. The Gaussian PTL is always a soft-core model.

\subsection{Measuring the Regularity of Perturbed Lat- tices}

In this section, we consider a wireless cellular network with the BS placement modelled by the two PTL models introduced in Section 4.2. The normalized parameters, $\tilde{R}$ for uniform PTL and $\tilde{\sigma}$ for Gaussian PTL, are swept from 0 to 2 in order to change the regularity of the models. (Indeed, the produced spatial pattern appears as if it is a PPP when the normalized parameters are higher than or equal to 2.) For each model, we measure the amount of regularity using the three CoV-based metrics introduced in Chapter 2. For each particular $\tilde{R}$ and $\tilde{\sigma}$ values, we perform a MonteCarlo simulation with 1000 realizations, each with 500 BSs placed over a $1 \mathrm{~km}^{2}$ square area. The CoV-based metrics as a function of $\tilde{R}$ and $\tilde{\sigma}$ are shown in Figures 4.1, 4.2, and 4.3.

A good fit for the relationship between the $C_{\mathrm{D}}$ metric and normalized parameters $(\tilde{R}$ and $\tilde{\sigma})$ of the uniform and Gaussian PLT is

$$
C_{\mathrm{D}} \approx 1-e^{-2.17 \tilde{R}^{1.095}}
$$




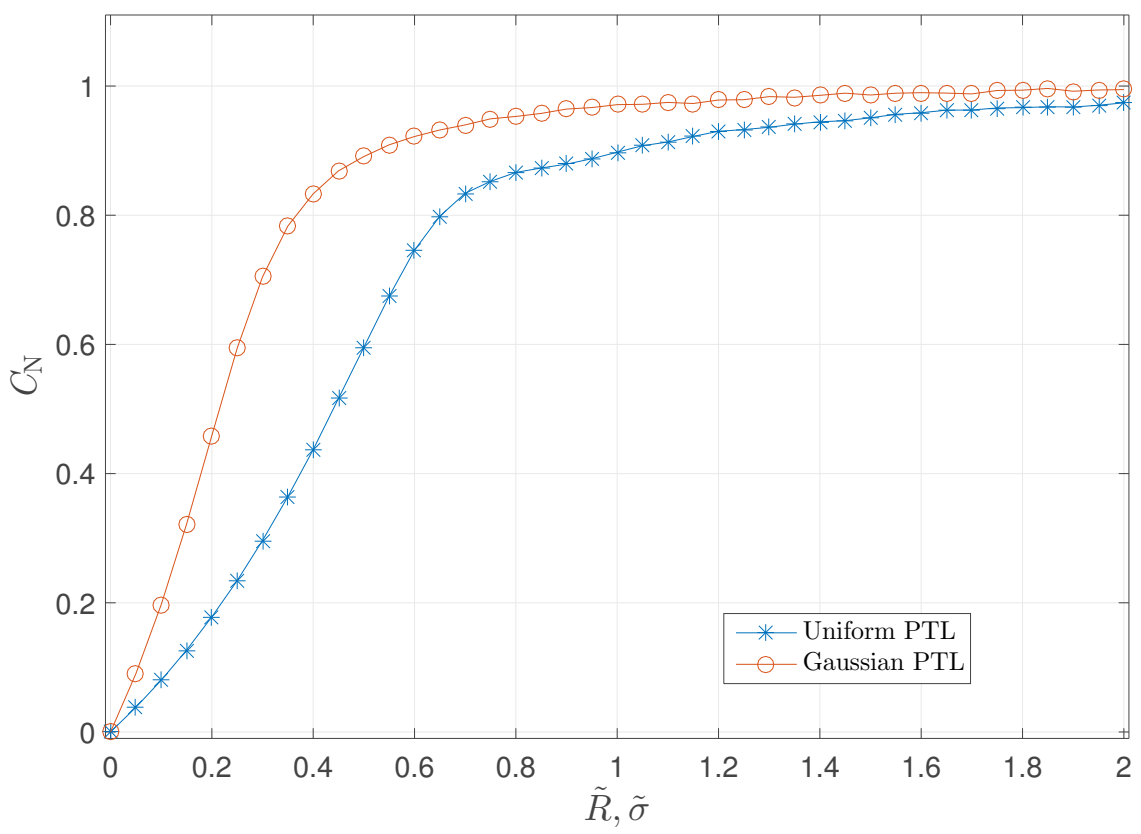

Figure 4.1: The normalized $\mathrm{CoV}$ of the distance to the nearest neighbour as a function of the normalized perturbations.

$$
C_{\mathrm{D}} \approx 1-e^{-4.189 \tilde{\sigma}^{1.0465}}
$$

As the perturbation parameters increase, the CoV-based metrics converge to 1 and the two models converge to the PPP. In other words, both models are able to span the whole range between the $\mathrm{TL}\left(C_{\mathrm{N}}=C_{\mathrm{V}}=C_{\mathrm{D}}=0\right)$ and, asymptotically, the $\operatorname{PPP}\left(C_{\mathrm{N}}, C_{\mathrm{V}}, C_{\mathrm{D}} \rightarrow 1\right)$. A particular spatial pattern has different $C_{\mathrm{N}}, C_{\mathrm{V}}$, and $C_{\mathrm{D}}$ values. Figure 4.4 shows the parametric curves of the relationships between the CoV-based metrics. 


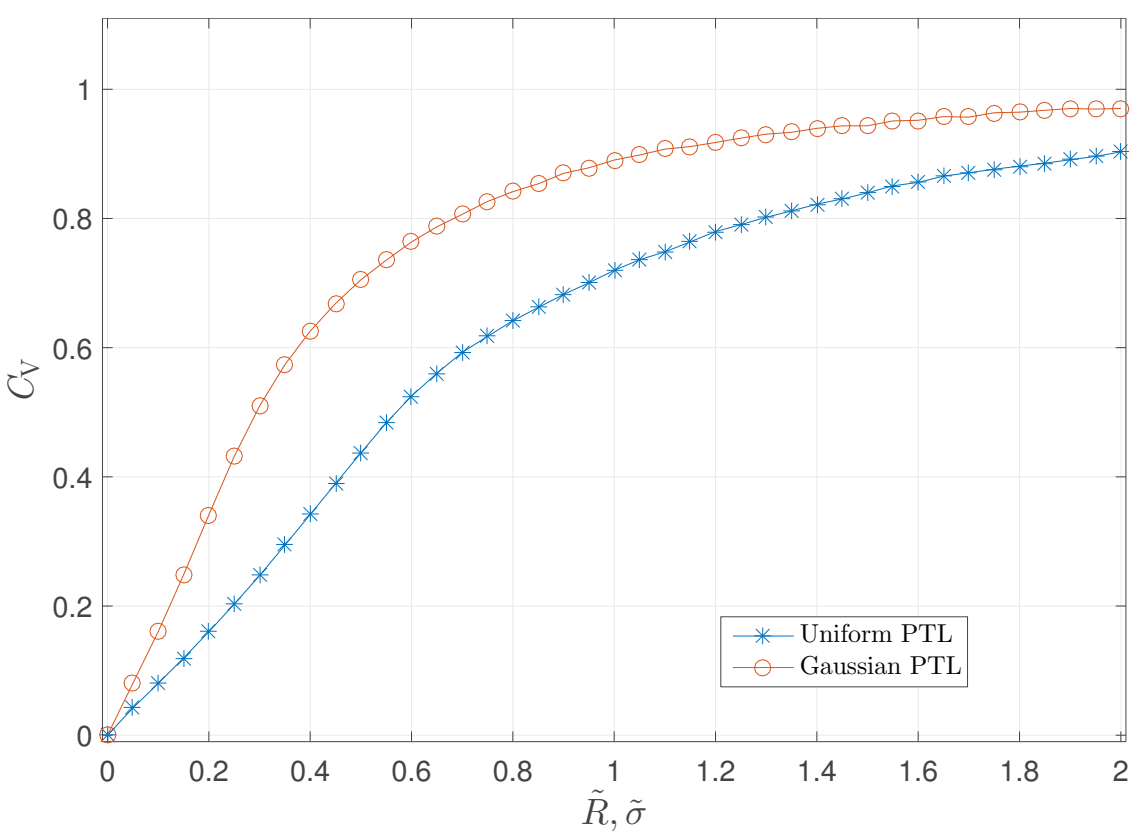

Figure 4.2: The normalized $\mathrm{CoV}$ of the areas of the Voronoi tessellation cells as a function of the normalized perturbations.

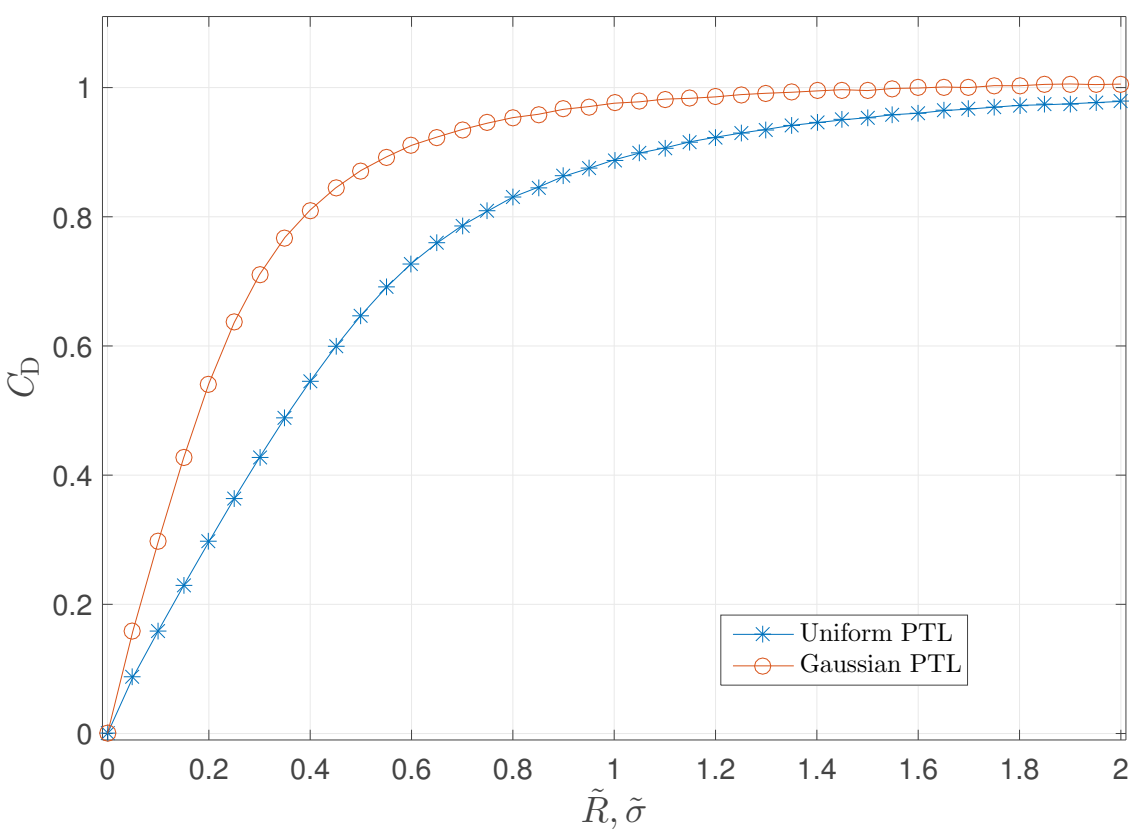

Figure 4.3: The normalized $\mathrm{CoV}$ of the lengths of the Delaunay triangulation edges as a function of the normalized perturbations. 


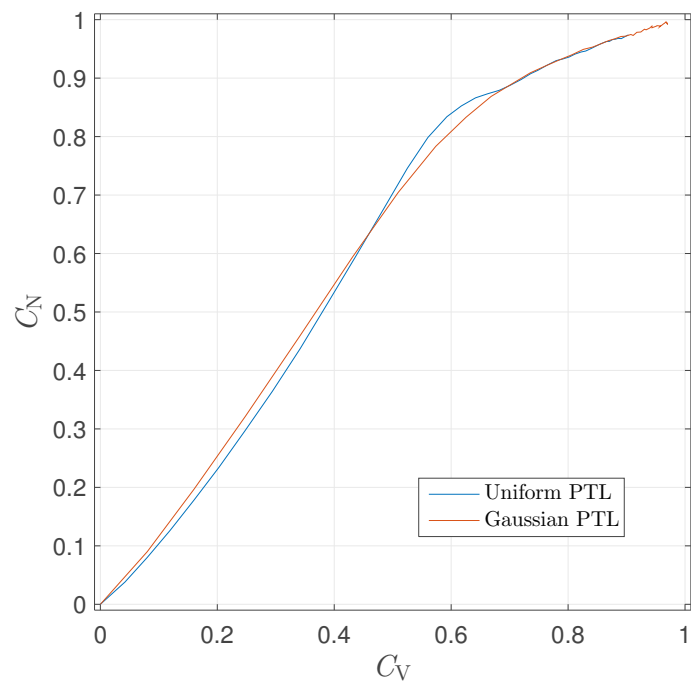

(a)

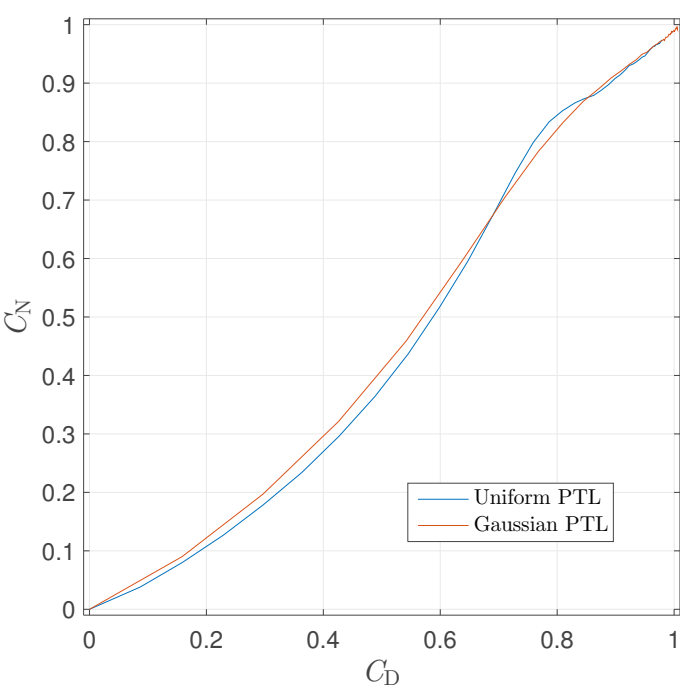

(b)

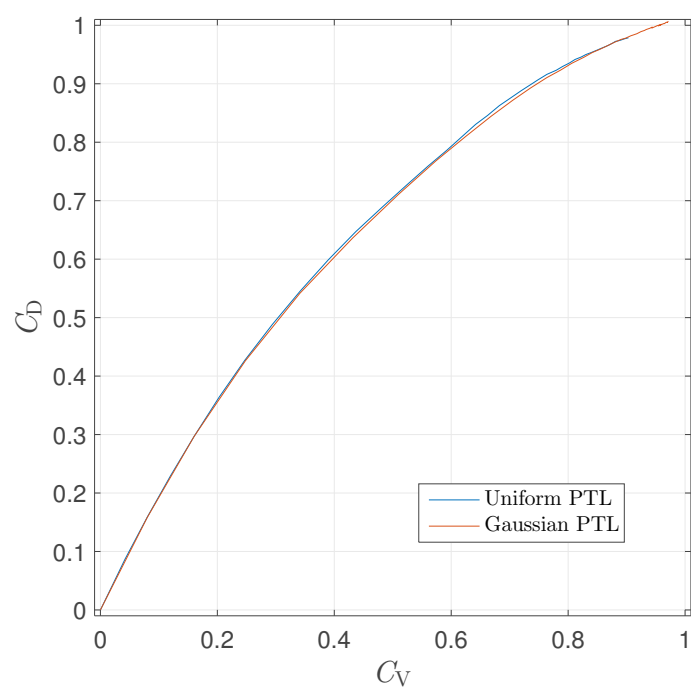

(c)

Figure 4.4: The relationship between different $\mathrm{CoV}$-based $\left(C_{\mathrm{N}}, C_{\mathrm{V}}\right.$, and $\left.C_{\mathrm{D}}\right)$ metrics of different PTL models. 


\subsection{Mapping Between Uniform PTL and Gaussian PTL Models Using the CoV-Based Metrics}

\subsubsection{Mapping}

In a novel framework, we use the CoV-based metrics as an intermediate step for mapping between the uniform PTL and the Gaussian PTL. Using the simulation results from the previous section, for each CoV-based metric at a particular value, we find the corresponding internal parameter values $\tilde{R}$ and $\tilde{\sigma}$, for the uniform PTL model and the Gaussian PTL model, respectively. Parametrized curves describing the relationship between $\tilde{R}$ and $\tilde{\sigma}$ using the three CoV-based metrics are shown in Figure 4.5. We find a good approximate fitting for all the three parametrized curves to be

$$
\tilde{\sigma} \approx 0.53 \tilde{R}
$$

This means that a spatial pattern generated by the uniform PTL with any pertur-

bation value $\tilde{R}$ is almost equivalent in terms of regularity to another spatial pattern generated by the Gaussian PTL with perturbation value $\tilde{\sigma}=0.53 \tilde{R}$. Moreover, the two spatial patterns — when they are used to model BS locations - are also almost equivalent in terms of the network performance, as we will show in the next subsection.

\subsubsection{Downlink Coverage Probability}

We now validate that a pair of spatial patterns generated by uniform PTL and Gaussian PTL have similar network performance and regularity (as measured using 


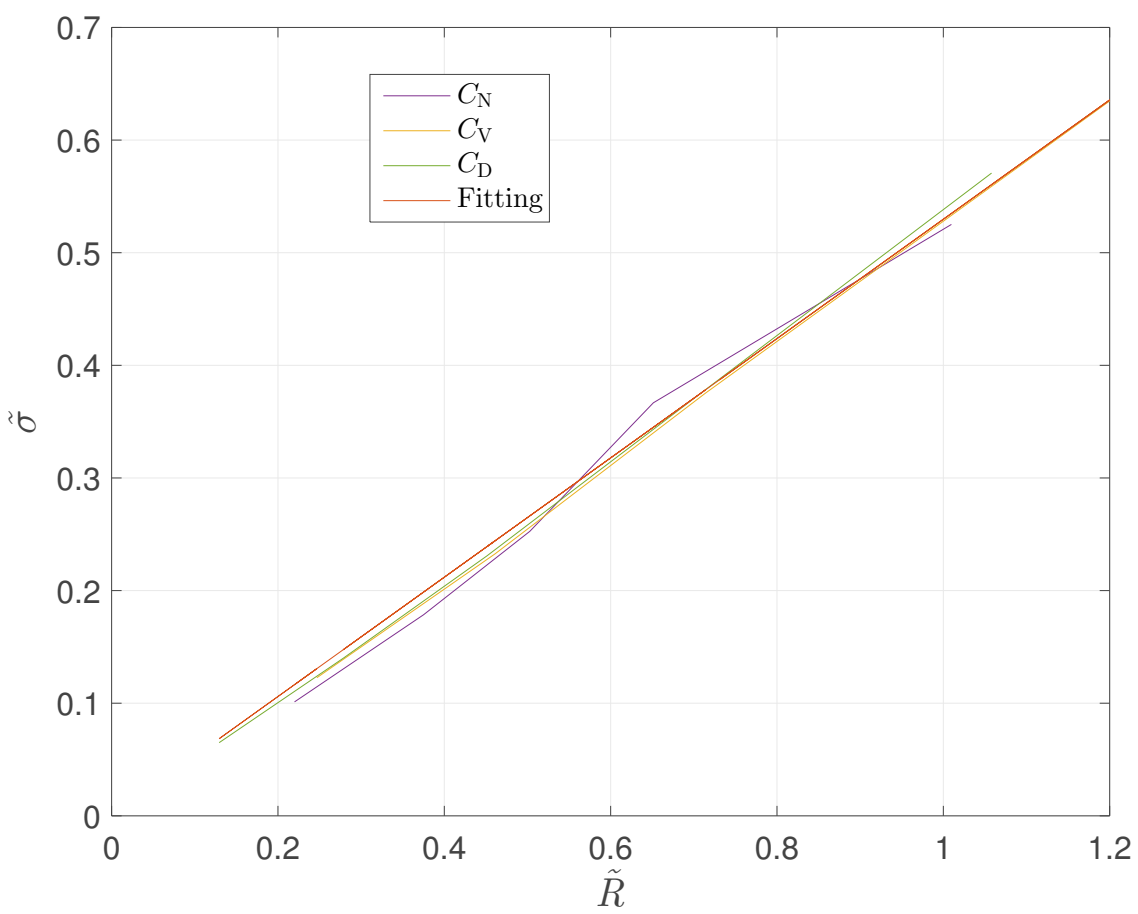

Figure 4.5: The relationship between $\tilde{R}$ and $\tilde{\sigma}$ that gives equal $\mathrm{CoV}$-metrics value.

the CoV-based metrics) if their internal parameters are simultaneously adjusted according to (4.6). A useful wireless network metric that we use to compare the network performance of different spatial deployments of BSs is the downlink coverage probability $P(\gamma)$, which is the probability that a typical user's signal-to-interference ratio (SIR) exceeds a threshold $\gamma$.

We consider a 100 macro BS (one tier) wireless network placed over a $1 \mathrm{~km}^{2}$ square area with simple network setup assumptions as follows. We consider two path-loss models: one with path-loss exponent $\alpha=3$ and lognormal shadowing of $X_{g}=6 \mathrm{~dB}$, and the other with $\alpha=4$ and $X_{g}=0 \mathrm{~dB}$. We will refer to these two path-loss models in Table I as $\mathrm{CH} 1$ and $\mathrm{CH} 2$, respectively. All the BSs transmit identical power, operate at the same frequency (the frequency reuse factor is 1 ), and are equipped with one antenna. 1000 mobile users are uniformly distributed over the network region. Each user is associated with its nearest serving BS. All the links experience independent 
Rayleigh fading with mean 1 . We ignore the thermal noise. We use a simple network setup, similarly to [13], because our emphasis is on the validation of the mapping between the two PTL models.

Figure 4.6 shows that uniform PTL and Gaussian PTL have similar downlink coverage probability when their parameters are matched according to (4.6). Figure 4.6 also shows that the coverage probability curves of the PPP and TL represent the lower and the upper network performance bounds, respectively.

Figures 4.7 and 4.8, each for a specific path-loss model, show in detail the difference between the coverage probability curves and the PPP coverage probability curve. As defined in [13, Def. 9], this SIR difference is the deployment gain $G\left(p_{t}\right)$, where $p_{t} \in(0,1)$ is the target coverage probability. Table I summarizes the comparison between the two PTL models matched according to (4.6). The gap $\Delta \mathrm{SIR}_{50 \%}$ is the $\mathrm{dB}$ difference in SIR between the coverage probability curves of the matched uniform PTL and the Gaussian PTL at a coverage probability of 50\%. The matched models have similar CoV-based metrics values and negligible $\Delta \mathrm{SIR}_{50 \%}$ for the two path-loss models. In fact, the difference in SIR between the matched uniform and Gaussian PTL is never more than $0.104 \mathrm{~dB}$.

\subsection{Concluding Remarks}

- We proposed a novel approach for mapping between uniform PTL and Gaussian PTL using CoV-based metrics as an intermediate step.

- After measuring the regularity of PTL models using three metrics, we found a simple relation between the internal parameters of these two PTL models in 


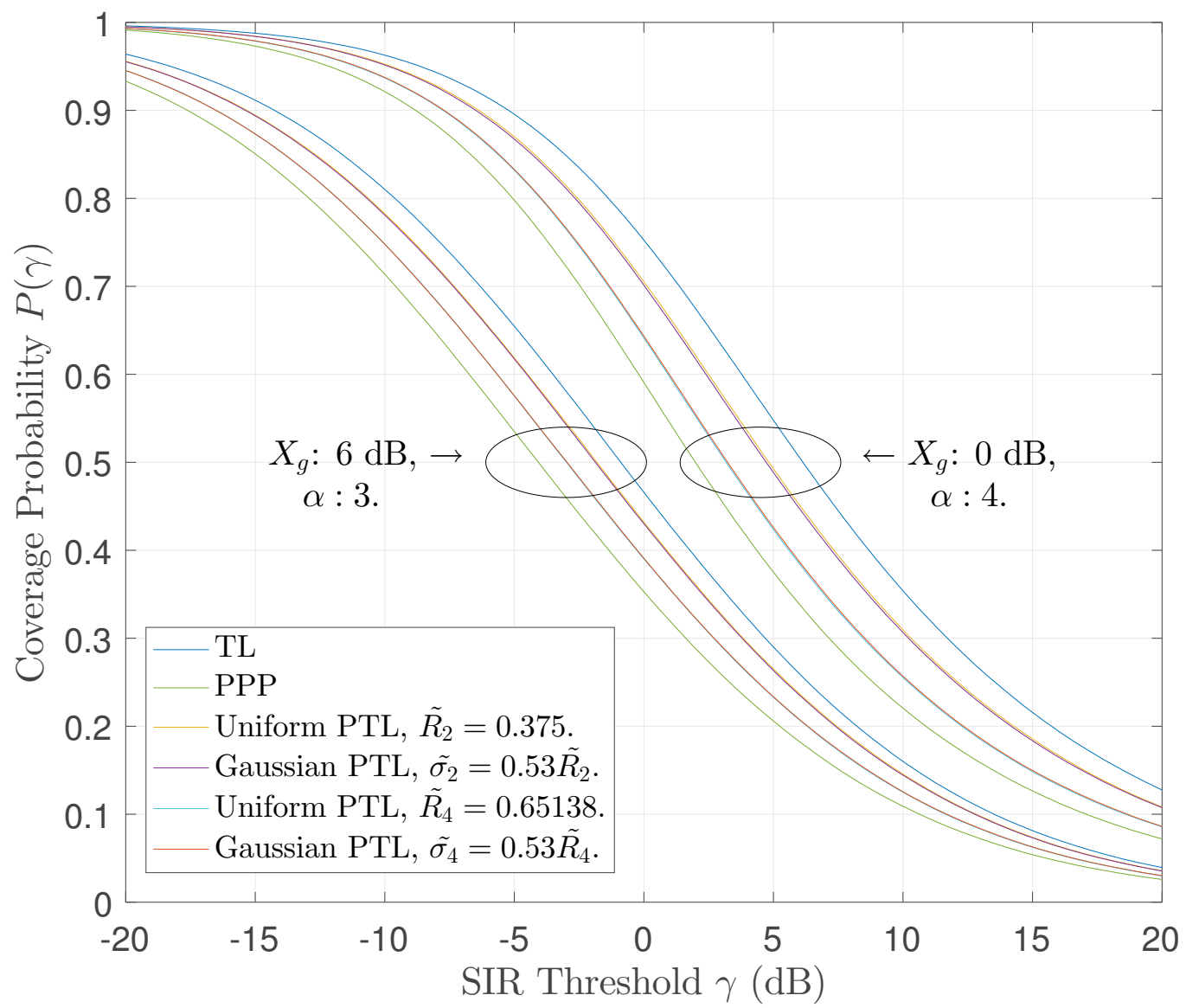

Figure 4.6: The coverage probability for different PTL models for different perturbation values and channel environments. 


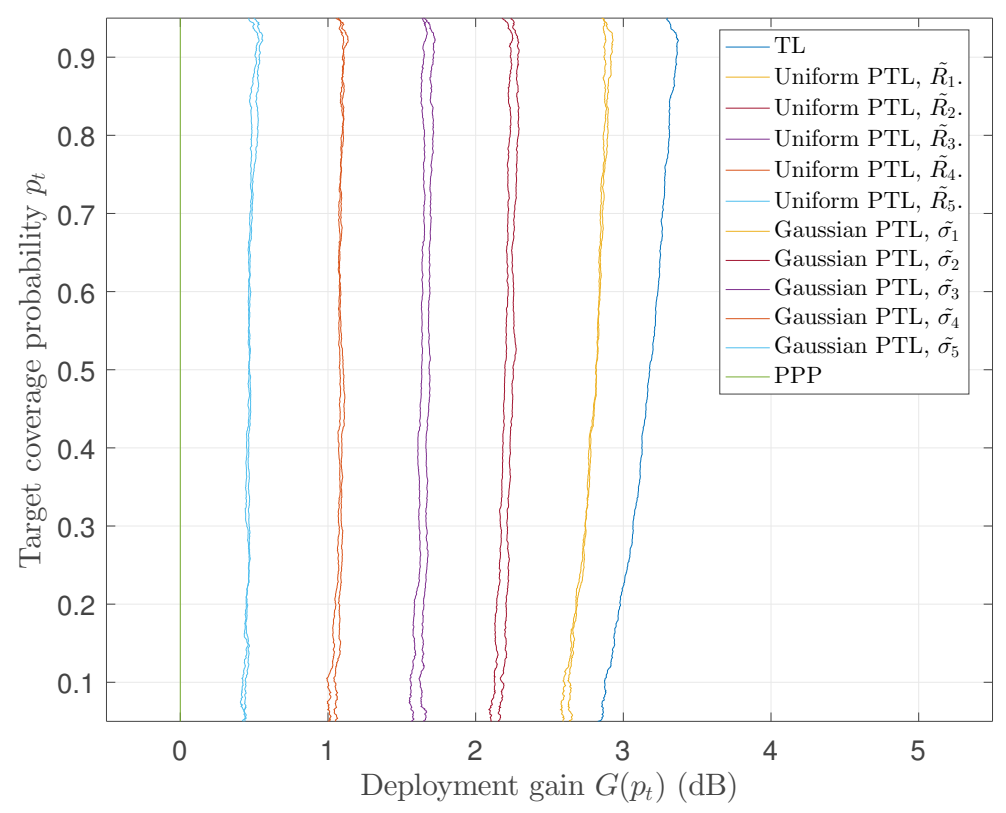

Figure 4.7: The difference in SIR between the curves of the coverage probability of different spatial patterns and the PPP coverage probability (the deployment gain). Path-loss model $\alpha=3$ and $X_{g}=6 \mathrm{~dB}$.

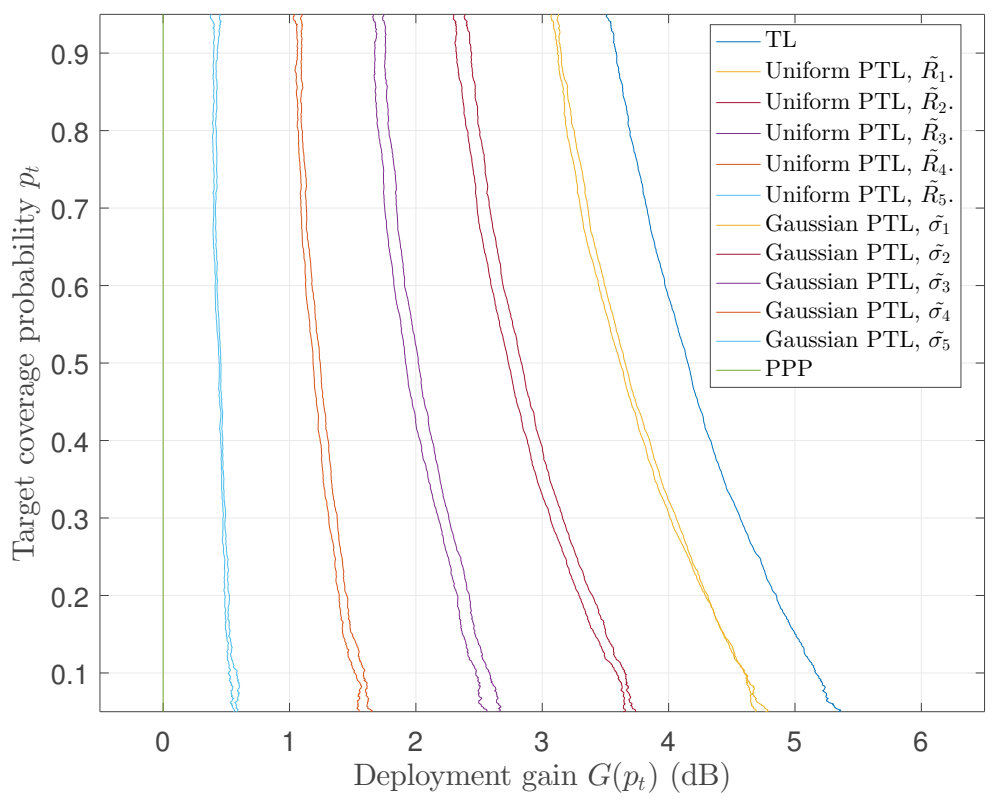

Figure 4.8: The difference in SIR between the curves of the coverage probability of different spatial patterns and the PPP coverage probability (the deployment gain). Path-loss model $\alpha=4$ and $X_{g}=0 \mathrm{~dB}$. 
Table 4.1: Summary of the comparison between the matched uniform PTL and Gaussian PTL.

\begin{tabular}{|l|l|l|l|l|l|l|}
\hline \hline & & $\tilde{R}_{1}$ & $\tilde{R}_{2}$ & $\tilde{R}_{3}$ & $\tilde{R}_{4}$ & $\tilde{R}_{5}$ \\
& $\tilde{R}$ & 0.219 & 0.375 & 0.503 & 0.651 & 1.000 \\
Uniform PTL & $C_{\mathrm{D}}$ & 0.323 & 0.517 & 0.650 & 0.760 & 0.888 \\
\hline \hline \multirow{2}{*}{ Gaussian PTL } & $C_{\mathrm{D}}$ & 0.341 & 0.539 & 0.663 & 0.763 & 0.885 \\
\hline \hline & & $\tilde{\sigma}_{1}$ & $\tilde{\sigma}_{2}$ & $\tilde{\sigma}_{3}$ & $\tilde{\sigma}_{4}$ & $\tilde{\sigma}_{5}$ \\
\hline \multirow{2}{*}{$\Delta \mathrm{SIR}_{50 \%}[\mathrm{~dB}]$} & $\mathrm{CH} 1$ & -0.003 & 0.055 & 0.051 & -0.023 & 0.003 \\
& $\mathrm{CH} 2$ & 0.038 & 0.104 & 0.101 & -0.057 & -0.013 \\
\hline \hline
\end{tabular}

order to generate two very similar spatial processes in terms of regularity and network performance. Therefore, considering only one type of PTL is enough since the other shows very similar performance.

- This chapter advocates modelling the placement of different types of BSs in HetNets using one of the PTL models, because of their simple and efficient implementation, their full regularity range (from the TL to the PPP), and their prevalence in industry and in recent wireless literature.

- Next chapter involves fitting RPP models to real BS location data to using the CoV-based regularity metrics and estimating the network performance based on the difference in the amount of regularity.

- Ultimately, we would like to describe the spatial structure of any wireless network using only two scalars: the density of the BSs and a regularity metric value. 


\section{Chapter 5}

\section{Towards the Best Metric for Measuring the Spatial Relationship among the Base Stations and Its Applications}

"There is no knowledgeable person, but there is another person with more knowledge."

- The Qur'an, 12:76

\section{$5.1 \quad$ Introduction}

In the previous chapters, we proposed a geometry-based scalar metrics to quantify the spatial regularity of the repulsive wireless networks. In this chapter, ${ }^{1}$ we aim to find the best metric among them. The results show that coefficient of variation (CoV) of the length of the corresponding edges of Delaunay triangulation is the most appropriate metric for measuring the regularity and it is robust for estimating the network performance. Using the best proposed regularity metric, this chapter develops new approaches for 1) approximating the performance of a repulsive network based on its amount of regularity and 2) fitting point processes to the spatial deployment of BSs.

Base station locations in wireless networks should be modelled via repulsive point processes with an amount of regularity that is tunable between that of a triangular

\footnotetext{
${ }^{1}$ This chapter is under review in IEEE Transactions on Wireless Communications [44].
} 
lattice and that of a homogeneous Poisson point process (PPP). However, the nonPoisson point processes (e.g., repulsive point processes including lattices) are much more complicated than the PPP to analyze due to the interaction among the points (BS locations). These models are much less investigated in the cellular networks literature due to the absence of a unified analytical framework to compute the coverage probability. For the non-Poisson networks, derivation of an exact closed-form expression for the coverage probability is either difficult or impossible to attain.

Interestingly, as observed in Figure. 4.6 as well as in the literature [8,13,45-48], the coverage probability curves of different BS deployments appear to be a horizontally shifted version of each other. This shift is a difference in the SIR threshold (in dB) along the x-axis. Guo and Haenggi [13] considered a PPP-deployed network as a reference and called this horizontal gap as the deployment gain. It is obtained mainly by deploying the BSs more regular than a PPP.

As a side note, the horizontal gap between the coverage curves is also called the "SIR gain", which is a more general term than the deployment gain since not only the deployment of the BSs is affected the horizontal gap, but also the channel model and the network parameters. The path loss exponent and fading also has some effect on the SIR gain. Other technologies such as employing MIMO (multiple-input and multipleoutput), sophisticated directional antennas, and interference cancellation methods may affect the SIR gain as well. Nevertheless, the SIR gain mainly depends on the spatial configuration of the network. That is why we need a metric that only depends on the spatial structure of the network such as the regularity gain, which is introduced in Section 5.4. Of course, the comparison of the performance of the networks with different spatial configuration must be made under the same network parameters and channel model. 


\subsubsection{Contribution}

The first contribution of this chapter is proposing a novel approach for approximating the performance of the repulsive cellular networks based on the amount of regularity of its BSs. Given a performance of a reference model (e.g., PPP-deployed network) is known, we proposed a simple approach to approximate the performance of different repulsive deployments of the BSs. In particular, we use the difference in the amount of regularity between the two deployments of BSs to estimate the performance of the unknown network when the performance of the other network is known. Second, we propose a novel approach for fitting RPP models to actual BS deployments. Given a real deployment of BSs, we used the $C_{\mathrm{D}}$ metric to know how to adjust the internal parameters of a particular point process to generate a spatial pattern equivalent to the real deployment. Finally, we show that the spatial structure of the actual deployment of BSs is locally regular.

The rest of this chapter is organized as follows. In Section 5.2, the performance metrics are presented. In Section 5.3, Strauss point process model is introduced and its regularity is measured using using the geometry-based regularity metrics. In Section 5.4, we quantify the deployment gain using the regularity metrics. Section 5.5 presents how the best geometry-based regularity metric can be used for fitting BSs to point processes. Section 5.6 demonstrates the local regularity of the spatial structure of the BSs. Finally, we draw the conclusions in Section 5.7. 


\subsection{Performance Metrics}

\subsubsection{Downlink Coverage Probability}

A useful wireless network metric that we use to compare the network performance of different spatial deployments of $\mathrm{BSs}$ is the downlink coverage probability $P(\gamma)$, which is the probability that a typical user's SI(N)R exceeds a threshold $\gamma$.

\subsubsection{The Deployment Gain}

Definition 1 (Deployment gain [13, Def. 9]): The deployment gain is the difference in the SIR between the coverage curves of the given point set and the PPP at a given target coverage probability $p_{t}$.

The deployment gain can be defined as [45]

$$
G\left(p_{t}\right) \triangleq \frac{\bar{F}_{\mathrm{SIR}}^{-1}\left(p_{t}\right)}{\bar{F}_{\mathrm{SIR}_{\mathrm{ref}}}^{-1}\left(p_{t}\right)}, \quad p_{t} \in(0,1),
$$

where $\bar{F}_{\mathrm{SIR}}^{-1}$ is the inverse of the CCDF of the SIR and $p_{t}$ is the target coverage probability. The average deployment gain $\hat{G}$ is approximately equal to the deployment gain at a target coverage probability $p_{t}=0.5$, i.e., $\hat{G} \approx G(0.5)[13]$. Throughout this thesis, we focus on the average deployment gain. 


\subsection{Strauss Point Process as Model for Base Sta- tion Placement}

\subsubsection{Strauss Process}

The Strauss process is an important class of Gibbs processes where it is obtained by shaping the distribution of PPP using a density function of the form

$$
f(\mathrm{X})=c \beta^{n(\mathrm{X})} \gamma^{s(\mathrm{X})}
$$

where $n(\mathrm{X})$ is the number of the points in $\mathrm{X}, s(\mathrm{X})$ is the number of point pairs that are closer than a hard-core distance $r$ apart. $0 \leq \gamma \geq 1$ is the interaction parameter. $\beta>0$ is a parameter and $c>0$ is a normalization constant. While the case $\gamma=1$ produces a PPP with intensity $\lambda_{\mathrm{P}}=\beta$, the case $\gamma=0$ gives Poisson hard-core process (PHCP). We found a good fitting for PHCP density ratio, which is

$$
\frac{\lambda}{\lambda_{\mathrm{P}}}=\frac{1-e^{-2.25 \tilde{r}^{1.73}}}{2.25 \tilde{r}^{1.73}},
$$

where $\tilde{r}=r \sqrt{\lambda_{\mathrm{P}}}$ is the normalized hard-core distance.

\subsubsection{Measuring the Regularity of the Strauss Point Process Models}

We consider wireless cellular networks where the locations of the BSs are modelled using the Strauss point process. In this section, the density of the point processes is 500 points (BSs) placed over a $1 \mathrm{~km}^{2}$ square area. Similar to the previous two chapters, we sweep the internal parameters of the Strauss point process and measure 


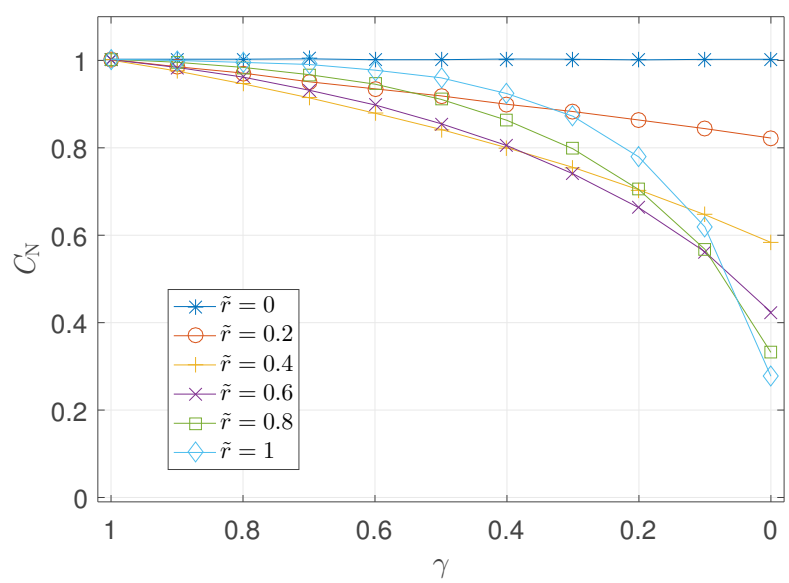

(a)

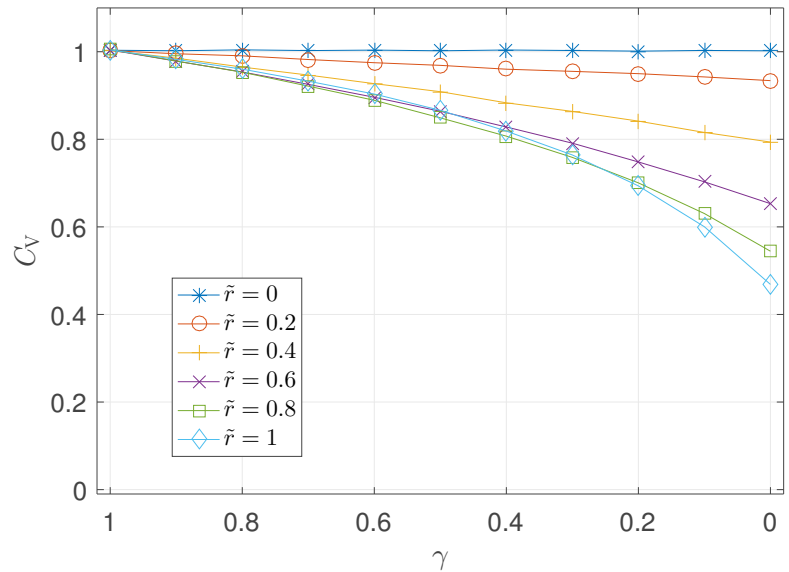

(b)

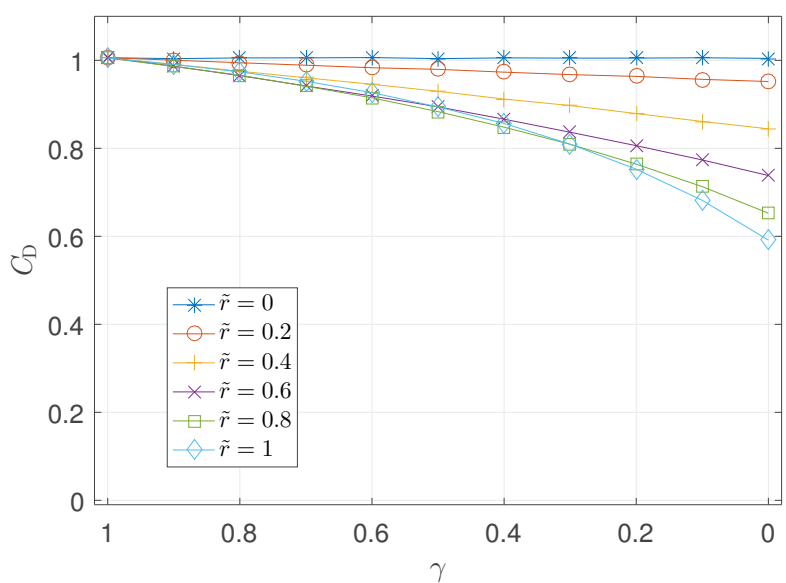

(c)

Figure 5.1: The normalized $\mathrm{CoV}$ of (a) the distance to the nearest neighbour $\left(C_{\mathrm{N}}\right)$, (b) the areas of the Voronoi tessellation cells $\left(C_{\mathrm{V}}\right)$, and $(\mathrm{c})$ the lengths of the Delaunay triangulation edges $\left(C_{\mathrm{D}}\right)$ as a function of the interaction parameter and the normalized hard-core distance $\tilde{r}$ for Strauss process. 
its regularity using the $\mathrm{CoV}$-based metrics defined in Chapter 2. At each particular increment in a parameter value, we perform a Monte-Carlo simulation with 1000 realizations.

Figure 5.1 shows the $\mathrm{CoV}$-based metrics as a function of the interaction parameter $\gamma$ and the normalized hard-core distance $\tilde{r}$ of the Strauss point process. The Strauss point process has a regularity floor similar to the hard-core point processes, investigated in Chapter 3. On the other hand, the PTL models can span the whole range between the TL $\left(C_{\mathrm{N}}, C_{\mathrm{V}}, C_{\mathrm{D}}=0\right)$ and, asymptotically, the PPP $\left(C_{\mathrm{N}}, C_{\mathrm{V}}, C_{\mathrm{D}} \rightarrow 1\right)$, as shown in Chapter 4 (Figs. 4.1-4.3). Clearly, the PTL models have full regularity range advantage unlike the Strauss and hard-core point processes. Therefore, the PTL is still our model of choice.

\subsection{Quantifying the Deployment Gain Using the Regularity Metrics}

As mentioned before, the SIR distributions of different cellular networks have similar shape regardless of the underlying spatial configuration of the BSs. Consequently, the SIR distribution of an arbitrary (real or simulated) repulsive BS deployment can be estimated relative to a known SIR distribution of a reference model. That is to say; knowing the SIR distribution of a reference model, we can determine the SIR distribution of an arbitrary network by computing its average deployment gain through the regularity difference (regularity gain) and then shift the SIR curve of the reference model accordingly. One point on the CCDF distribution is enough to determine the whole graph since all curves are similar [8,13,45-48]. 
This approach obviates the need to tackle less tractable models or to repeat cumbersome simulations. It brings the closed-form expressions of the Poisson point process to non-Poisson real deployments after add/subtract (in dB) a constant to reflect the average deployment gain.

Definition 2 The regularity gain ${ }^{2}$ is the difference in the amount of the spatial regularity/irregularity between a PPP-deployed network and an arbitrary network deployment.

Formally, we define the regularity gain, when the $C_{\mathrm{D}}$ metric is used for the measurement, as

$$
\Gamma_{\mathrm{D}}=C_{\mathrm{D}}^{\mathrm{ref}}-C_{\mathrm{D}}
$$

Not unexpectedly, we consider a PPP-deployed network (i.e., Poisson deployment of BSs) as a reference model since many studies chose it as a reference model.

In this section, we first examine three $\mathrm{CoV}$-based metrics to know which is the most robust to be used for approximating and comparing the performance of cellular networks of different spatial deployments. Then, we use the difference in regularity between two spatial sets of BSs, as measured using the most robust metric, to estimate the performance of one set of BSs if the performance of the other set is known.

\subsubsection{Data}

Here we measure the average deployment gain and the regularity of actual (realworld) and artificial (simulated) spatial patterns of BS.

\footnotetext{
${ }^{2}$ We mimicked the name of deployment gain in [13]. The regularity gain can also be called the repulsion gain.
} 
The data of the actual deployment of the BSs is obtained from the Sitefinder database, ${ }^{3}$ which represents real BS developments in the UK. The data is publicly released by Ofcom - the communications regulator authority in the UK. In this chapter, we consider only the locations of macro BSs (GSM band $900 \mathrm{MHz}$ ) that belong to the operator Vodafone. From this data, we selected 36 small sets of BSs that represent the locations of real spatial deployments of BSs in different regions. These sets are selected from the database as follows. We assumed that each point (BS location) in the database to be a centre of a rectangular area. ${ }^{4}$ Then, we extracted the points within each rectangular area - including the BS at the centre - and denoted it as a set of BSs. Since the number of the BSs in the rectangular areas is not equal because of the change in the geographical location and the underlying population density, we only select the rectangular areas that have at least $60 \mathrm{BSs}$ inside. The average number of the BSs in a rectangular area is 64 and the maximum is 65 .

For the simulated data, we construct the artificial spatial patterns of BS locations using the point processes described in Section 3.2, Section 4.2, and Section 5.3. As in the previous section, we sweep with a fixed step size increment the parameters of the models to change the regularity of the produced spatial patterns. We measure the performance and the amount of regularity of these spatial patterns using the regularity metrics of Chapter 2.

\subsubsection{System Model}

We consider simple network setup assumptions as follows. All the BSs transmit identical power, operate at the same frequency (the frequency reuse factor is 1),

${ }^{3}$ The Sitefinder database of the locations of BSs is available at
http://www.ofcom.org.uk/static/sitefinder/Sitefinder-May-2012.zip
${ }^{4}$ We converted the geographic coordinates (latitude, longitude) of the BSs to the Cartesian co-
ordinates $(x, y)$ before using them in the simulation.


and are equipped with one antenna. Mobile users are uniformly distributed over the network region. Each user is associated to its nearest serving BS. All the links experience independent Rayleigh fading with mean 1 . We ignore the thermal noise. We consider different combinations for the path-loss model parameters as follows. The path-loss exponent $\alpha$ and the lognormal shadowing of $X_{g}$ dB pairs are $(2.5,6$ $\mathrm{dB}),(2.5,0 \mathrm{~dB}),(3,6 \mathrm{~dB}),(3,0 \mathrm{~dB}),(3.5,6 \mathrm{~dB}),(3.5,0 \mathrm{~dB}),(4,6 \mathrm{~dB}),(4,0 \mathrm{~dB})$, and (4.5, $6 \mathrm{~dB})$. We consider rectangular geographical areas of $1500 \mathrm{~m} \times 1050 \mathrm{~m}$. Users are uniformly distributed within a central area of $900 \mathrm{~m} \times 630$ to reduce the edge effect on the network performance calculation. We execute the experiment for 1000 Monte Carlo simulation runs.

\subsubsection{Results}

In Figs. 5.2-5.4, we draw parametric curves of the relationship between the amount of the regularity and the average deployment gain of different simulated (modelled using point processes presented in Section 3.2, Section 4.2, and Section 5.3) and actual deployments of BSs for different channel parameters.

On each of these figures, we also plot a linear regression (greed solid line) for the discrete points that represent the average deployment gain of the real spatial deployments of BS sets.

Note that, for the SP, we sketch the average deployment gain as a function of the interaction parameter $\gamma$ at a fixed normalized hard-core distance $\tilde{r}=0.5$.

Figs. 5.2-5.4 show that regardless of the metric type, the repulsive point processes of the same variety have the same network performance (average deployment gain) when they have the same amount of regularity. The soft-core and the hard-core 


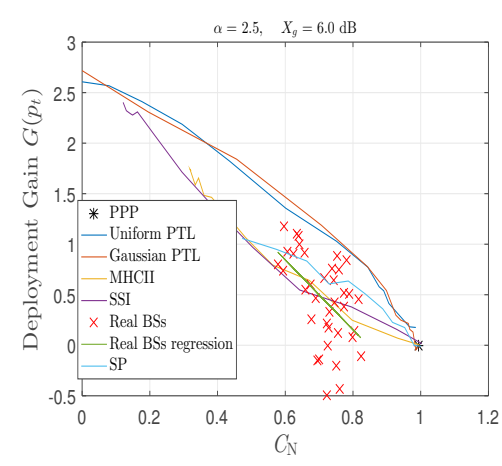

(a) $\alpha=2.5 \sigma=6$

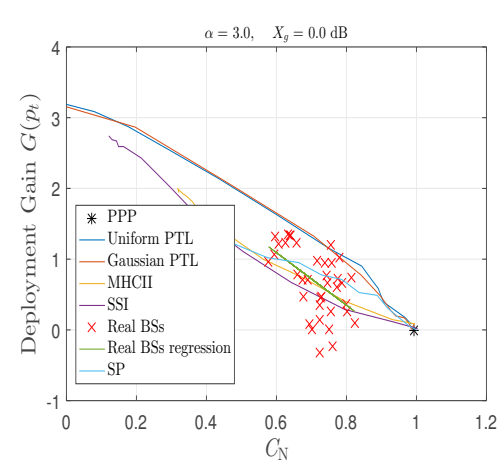

(d) $\alpha=3 \sigma=0$

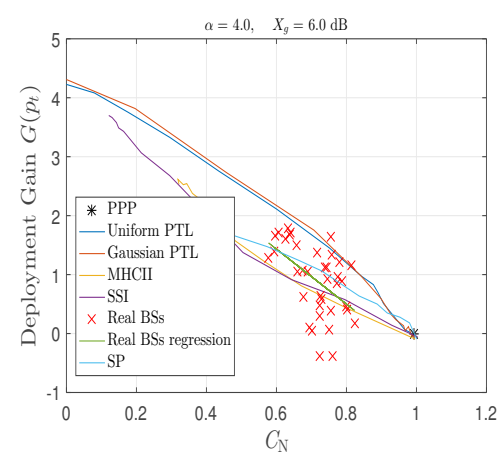

(g) $\alpha=4 \sigma=6$

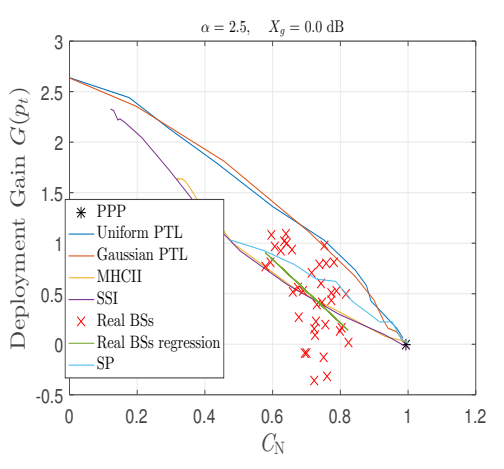

(b) $\alpha=2.5 \sigma=0$

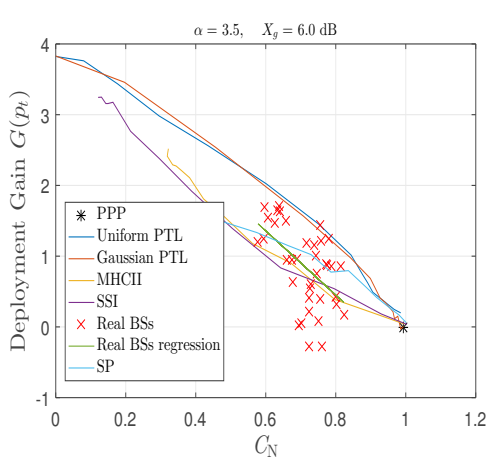

(e) $\alpha=3.5 \sigma=6$

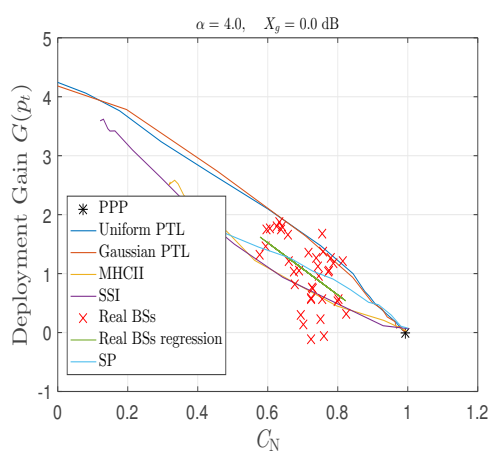

(h) $\alpha=4 \sigma=0$

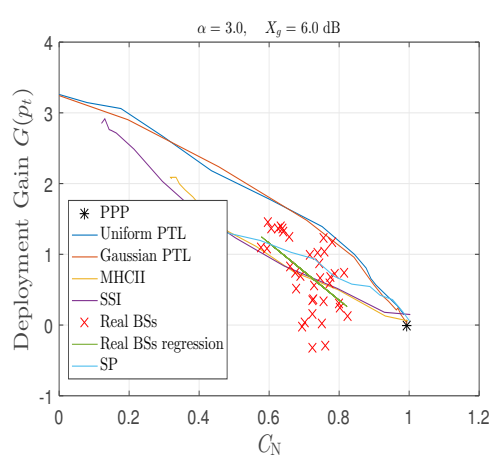

(c) $\alpha=3 \sigma=6$

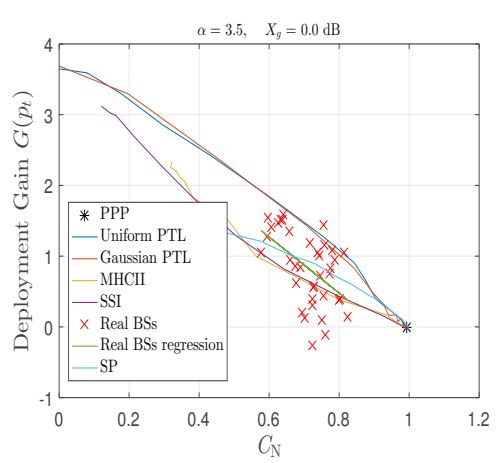

(f) $\alpha=3.5 \sigma=0$

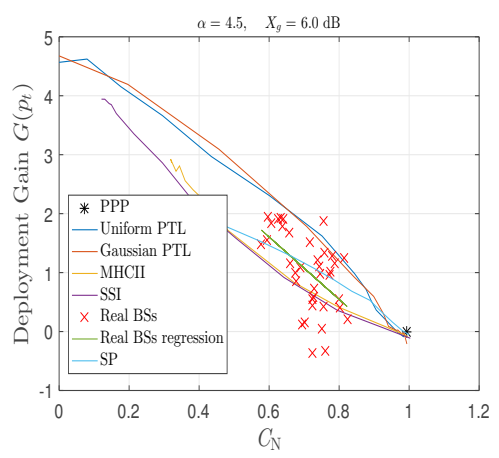

(i) $\alpha=4.5, \sigma=6$

Figure 5.2: The relationship between the regularity as measured using the $C_{\mathrm{N}}$ metric and the deployment gain $G\left(p_{t}\right)$ at a target coverage probability $p_{t}=$ 0.5 of different simulated and actual deployments of BSs for different channel parameters: The path-loss exponent $\alpha$ and shadowing $X_{g}$. 


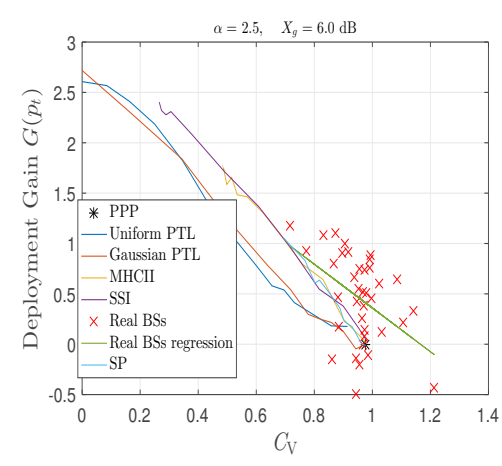

(a) $\alpha=2.5 \sigma=6$

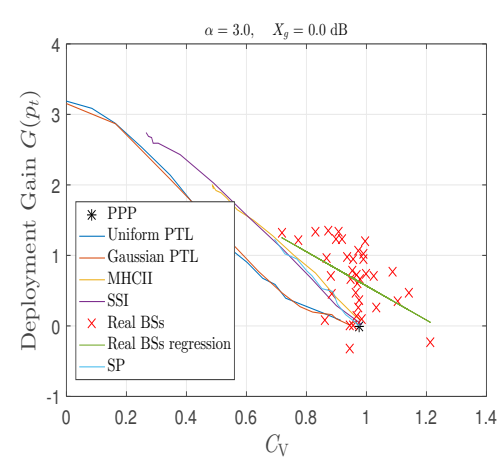

(d) $\alpha=3 \sigma=0$

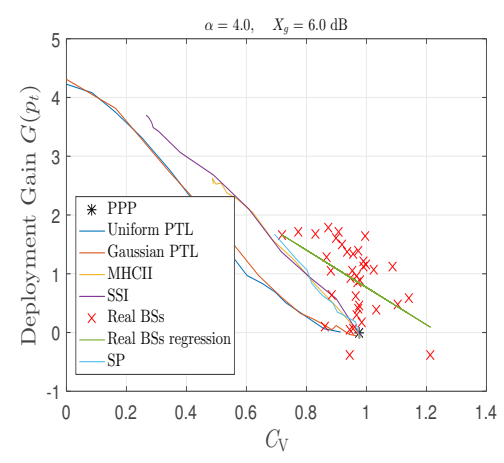

(g) $\alpha=4 \sigma=6$

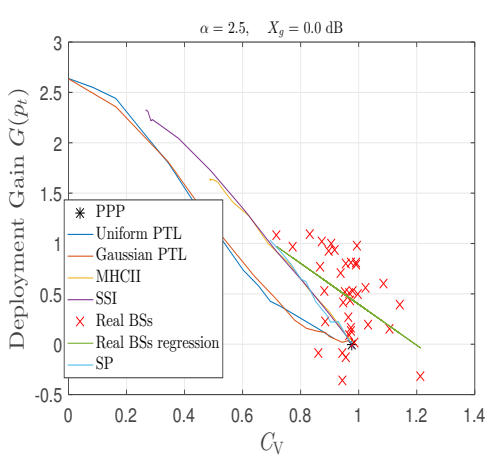

(b) $\alpha=2.5 \sigma=0$

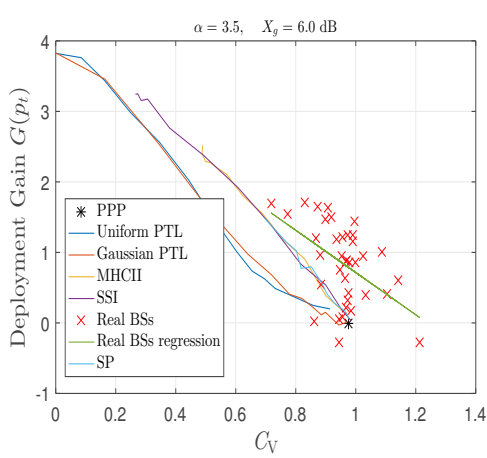

(e) $\alpha=3.5 \sigma=6$

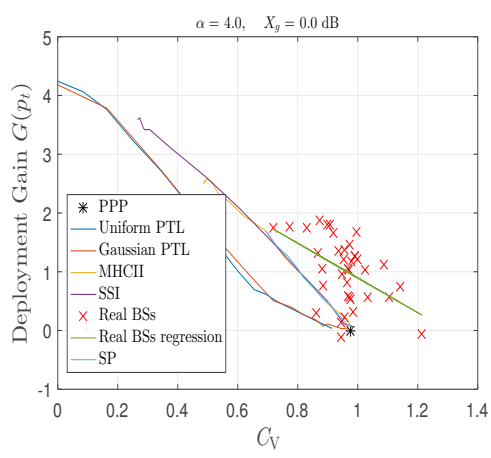

(h) $\alpha=4 \sigma=0$

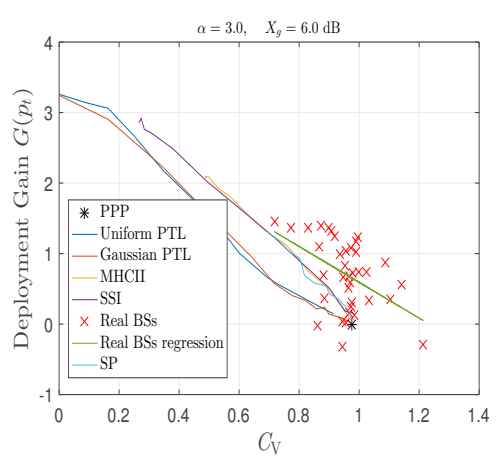

(c) $\alpha=3 \sigma=6$

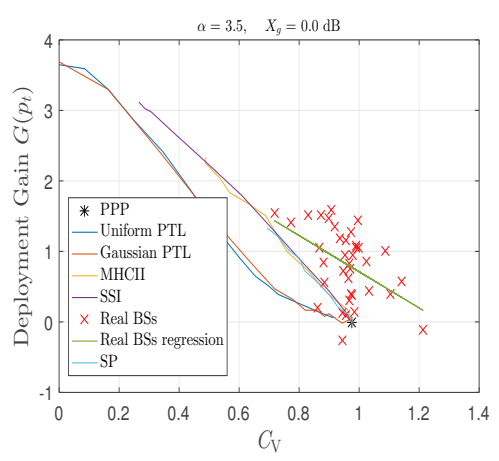

(f) $\alpha=3.5 \sigma=0$

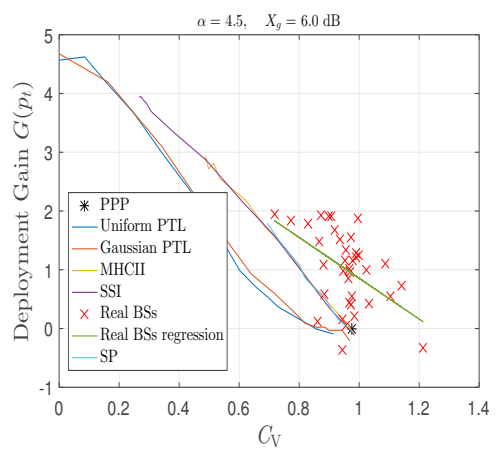

(i) $\alpha=4.5, \sigma=6$

Figure 5.3: The relationship between the regularity as measured using the $C_{\mathrm{V}}$ metric and the deployment gain $G\left(p_{t}\right)$ at a target coverage probability $p_{t}=$ 0.5 of different simulated and actual deployments of BSs for different channel parameters: The path loss exponent $\alpha$ and shadowing $X_{g}$. 


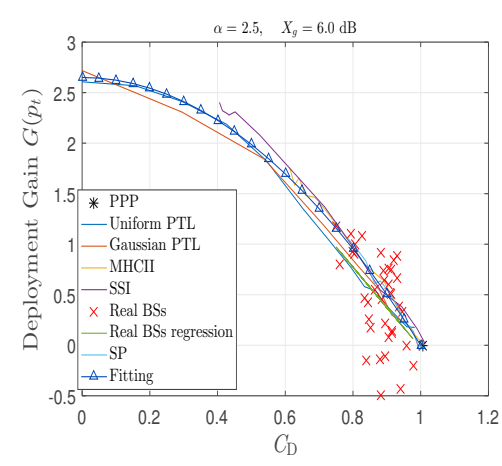

(a) $\alpha=2.5 \sigma=6$

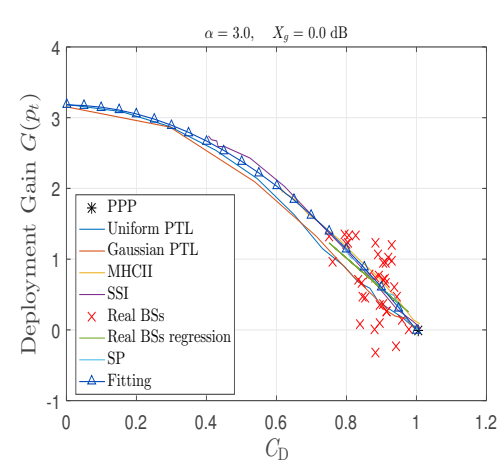

(d) $\alpha=3 \sigma=0$

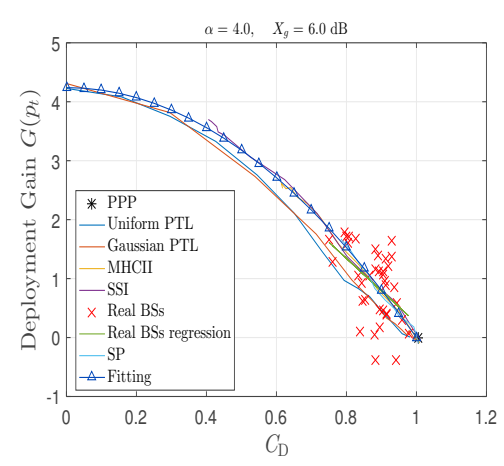

(g) $\alpha=4 \sigma=6$

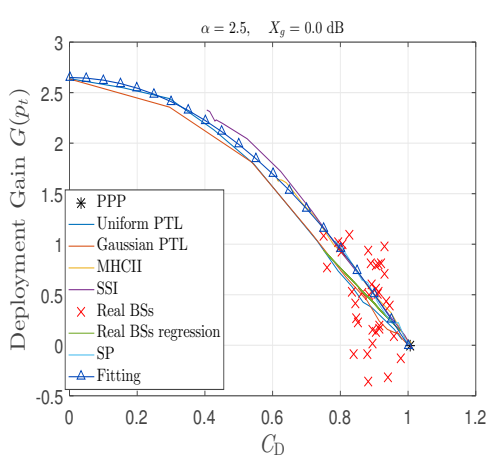

(b) $\alpha=2.5 \sigma=0$

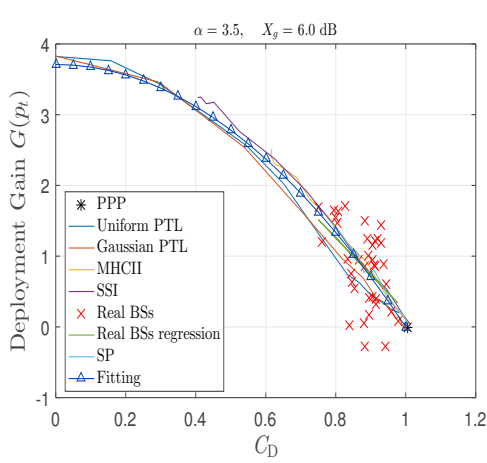

(e) $\alpha=3.5 \sigma=6$

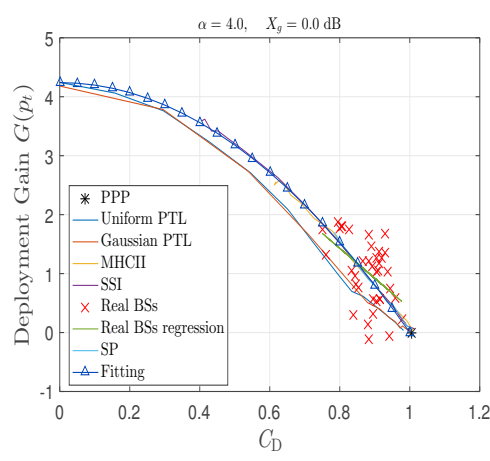

(h) $\alpha=4 \sigma=0$

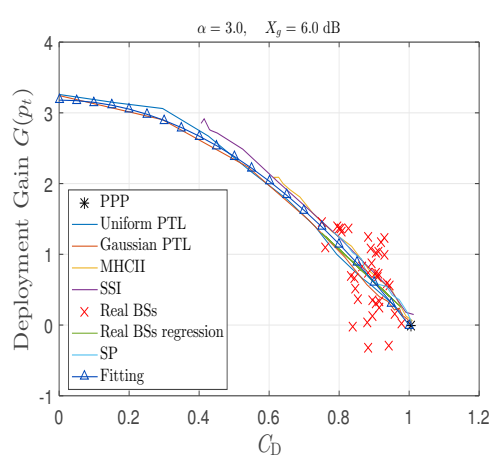

(c) $\alpha=3 \sigma=6$

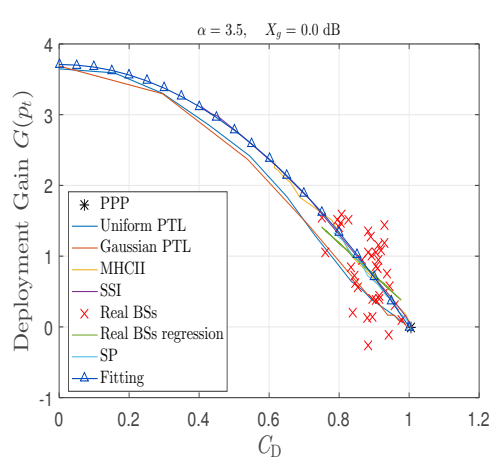

(f) $\alpha=3.5 \sigma=0$

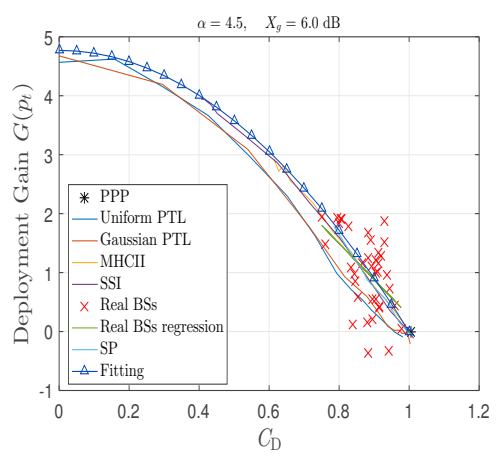

(i) $\alpha=4.5, \sigma=6$

Figure 5.4: The relationship between the regularity as measured using the $C_{\mathrm{D}}$ metric and the deployment gain $G\left(p_{t}\right)$ at a target coverage probability $p_{t}=$ 0.5 of different simulated and actual deployments of BSs for different channel parameters: The path-loss exponent $\alpha$ and shadowing $X_{g}$. 
point processes have slightly different network performance even when they have the same amount of regularity. When the regularity is quantified using the $C_{\mathrm{D}}$ metric, we observe that 1) the performance gap between the soft-core and the hard-core point processes is negligible, and 2) the performance of the two varieties of the RPPs is similar to the performance of the linear regression of the real BSs deployment. Therefore, the $C_{\mathrm{D}}$ metric is the most robust and the best indicator for the network performance. In Chapter 6 and Chapter 7 , we use the $C_{\mathrm{D}}$ metric to facilitate the planning of the next generation cellular networks: the cells switch-off (CSO) during the low traffic periods and deploying mobile on-demand BSs mounted on unmanned aerial vehicles (UAV-BSs).

We note that the effect of the shadowing on the deployment gain is negligible. Indeed, the shadowing only causes a right or left shift to all coverage curves including the baseline model (i.e., the PPP) as shown in Figure 4.6. In other words, the shadowing introduces almost equal shift to the coverage curves, but its effect on the deployment gain is negligible (within 0.1 to $0.2 \mathrm{~dB}$ difference), taking into consideration a PPP-deployed network of the same network parameter is considered as a reference. For the similar network configuration, the deployment gain depends on the spatial structure of the BSs and the path loss exponent.

For measurement using the $C_{\mathrm{D}}$ metric, a good approximation for the relationship between the deployment gain and the amount of regularity is

$$
G(0.5) \approx-c \alpha\left(C_{\mathrm{D}}\right)^{2}+c \alpha
$$

where $\alpha$ is the path-loss exponent and $c \approx 1.06$ is a constant. The value of the constant $c$ may slightly change depending on the simulation environment and the path-loss exponent. The chosen value for the constant $c$ is the average over all considered 
channel models.

For $\alpha=4$ and using (5.5), we can estimate that the performance of a repulsive network of an amount of regularity $C_{\mathrm{D}}=0.8$ (or, equivalently, regularity gain $\Gamma_{\mathrm{D}}=0.2$ ) is approximately $1.52 \mathrm{~dB}$ better than a PPP-deployed network of the same network configurations.

\subsection{Fitting Base Station Locations to Repulsive Point Processes}

In this section, we introduce a simple, yet accurate, approach for fitting point processes to locations of BSs in cellular networks. This approach uses the $\mathrm{CoV}$ based metrics as fitting tools. In particular, we consider fitting PTLs to spatial point patterns of real and simulated data using the $C_{\mathrm{D}}$ metric. The fitting procedure is as follows.

Step 1: Identify a bounded geographical region (window) of a real-world (or simulated) repulsive deployment of BSs. Then, measure its density and its regularity using the $C_{\mathrm{D}}$ metric.

Step 2: Choose a targeted point process, whether it is a soft-core or a hard-core model, that could achieve the measured regularity value. (In this section, we select the PTL models since they span all range of regularity.) Choosing the appropriate model requires in advance investigation of all candidate point processes with respect to their type and regularity range as measured using the CoV-based metrics.

Step 3: Finally, tune the internal parameters of the targeted model to generate 


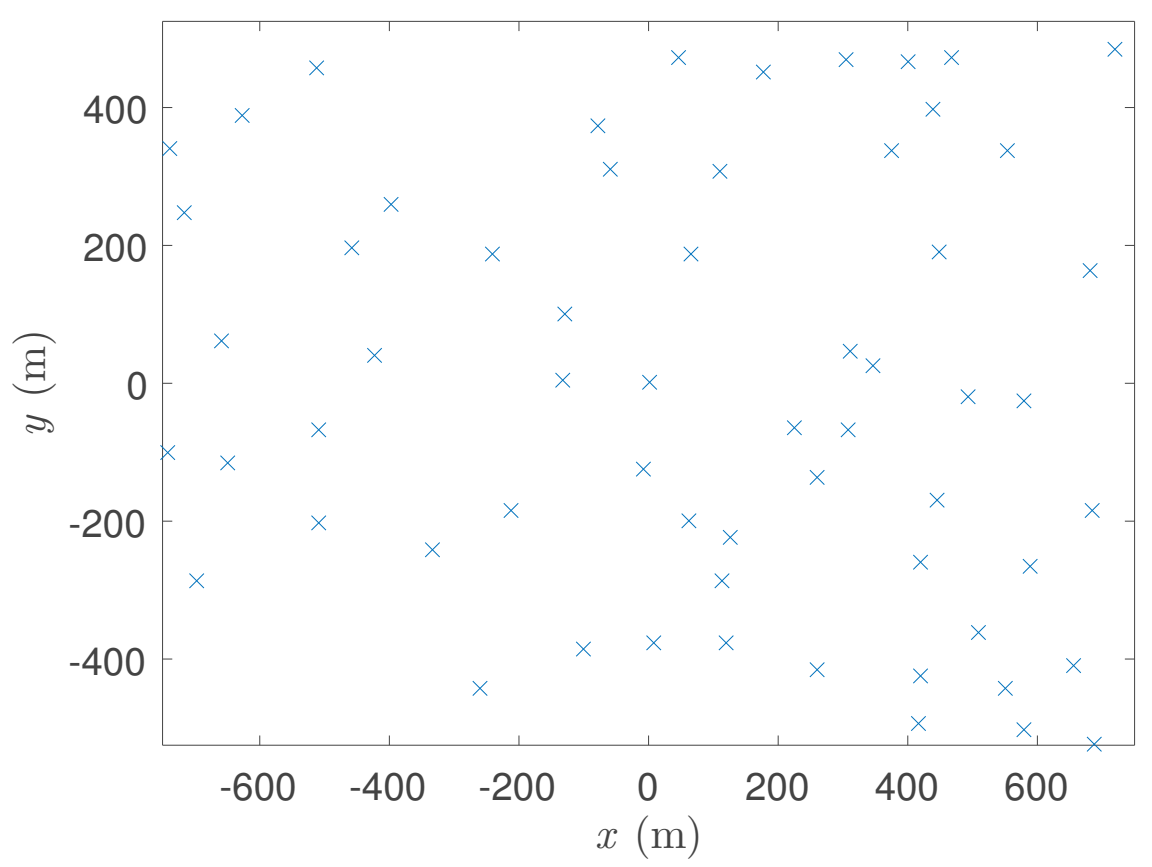

(a) Actual data

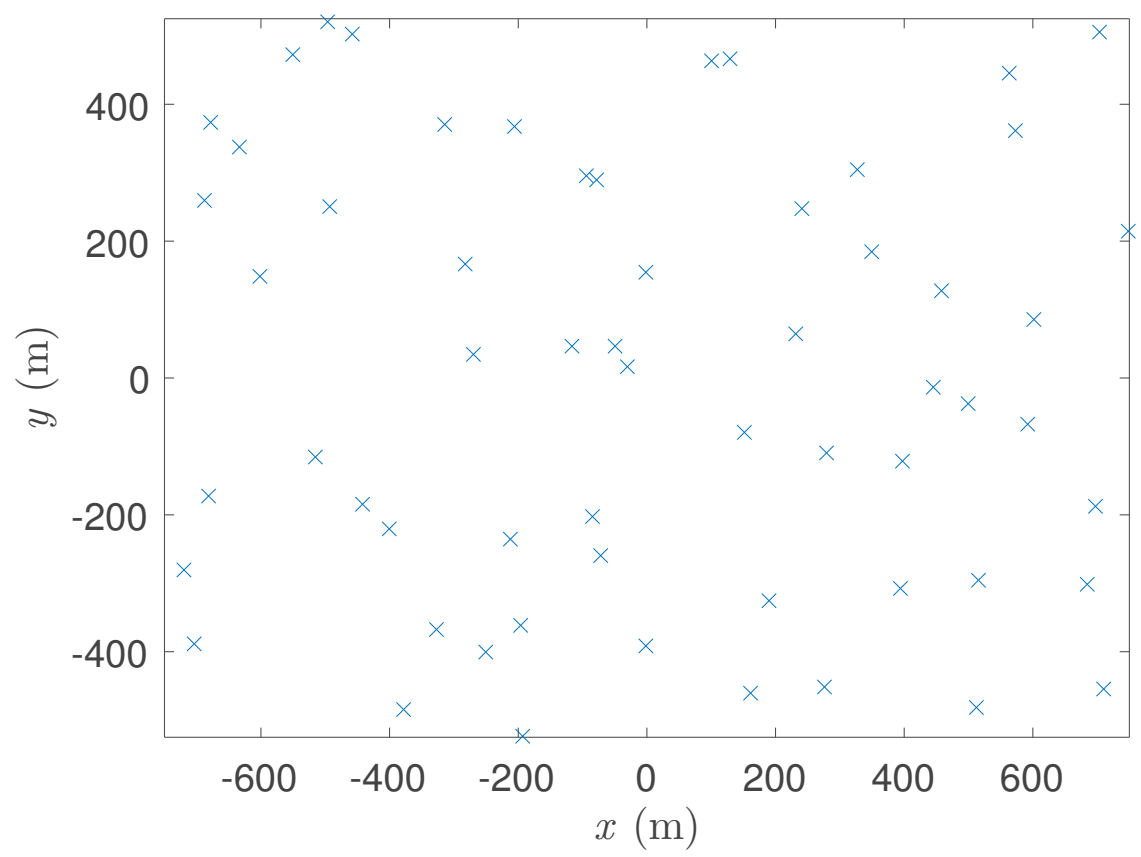

(b) Gaussian PTL

Figure 5.5: Two similar (the amount of regularity $C_{\mathrm{D}}=0.75$ and density $\lambda=38.73$ $\mathrm{BSs} / \mathrm{km}^{2}$ ) spatial deployments of BSs. (a) real (actual) data extracted from the Sitefinder database. It consists of 61 BSs distributed over $1500 \mathrm{~m} \times 1050 \mathrm{~m}$ region centred at $\left(51.5157^{\circ} \mathrm{N},-0.1395^{\circ} \mathrm{W}\right)$. (b) A realization using Gaussian PTL $(\tilde{\sigma}=0.3357)$. 


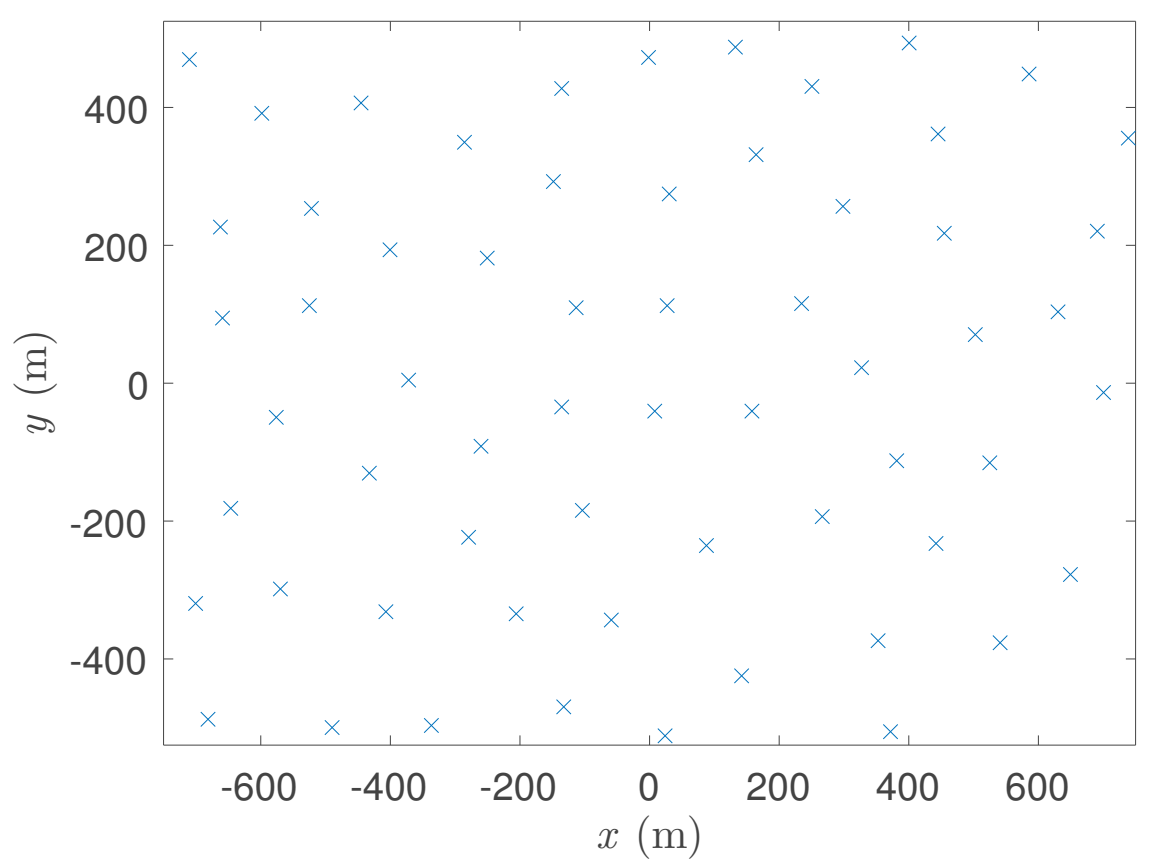

(a) Simulated data using SSI

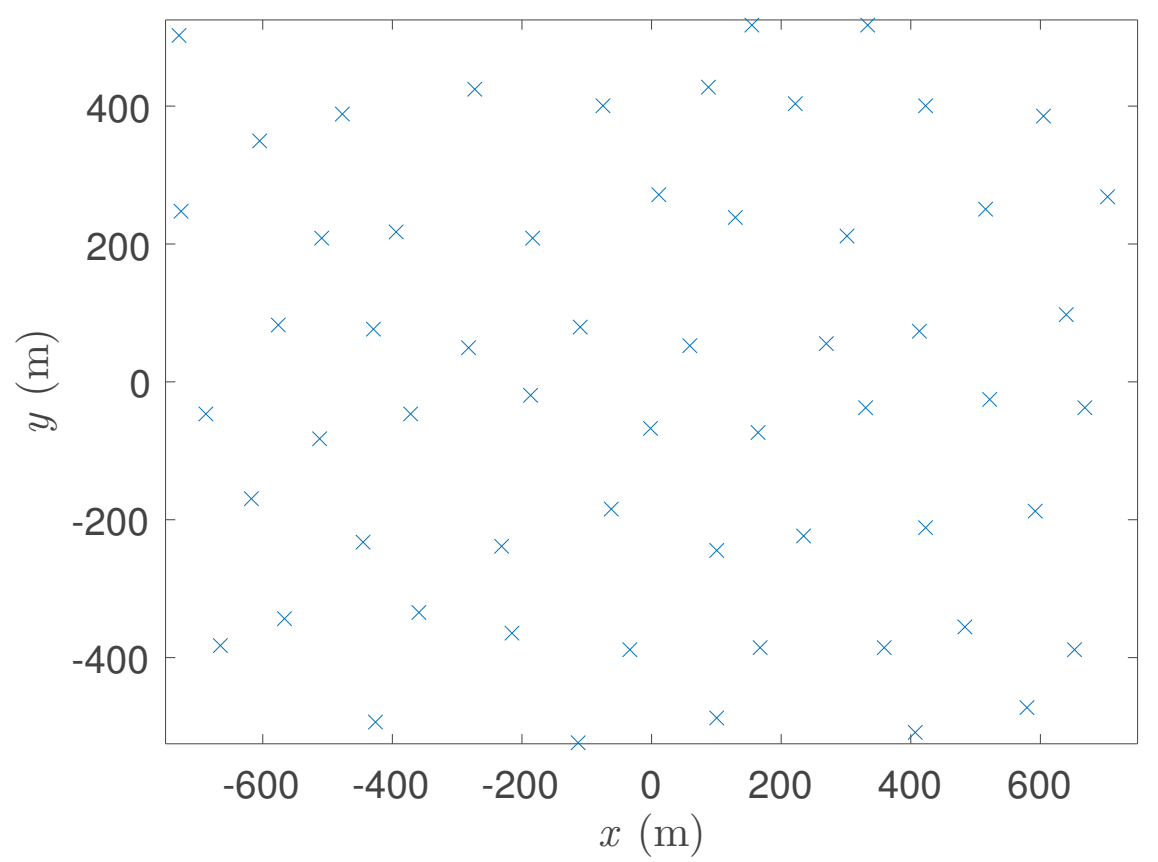

(b) A relaization using uniform PTL

Figure 5.6: Two similar (the amount of regularity $C_{\mathrm{D}}=0.38$ and density $\lambda=38.73$ BSs $/ \mathrm{km}^{2}$ ) spatial deployments of BSs. (a) Simulated data using SSI (hard-core distance $r=131.35 \mathrm{~m})$. (b) A realization using uniform PTL $(\tilde{R}=0.26)$. 
a spatial pattern that has equal regularity value and BSs density as the given deployment. A fast adjustment of the internal parameters of a model requires a prior knowledge about the relationship between them and the regularity value. (We investigated the relationship between the internal parameters and the regularity ranges of different RPPs in Section 5.3.2 and in the previous chapters.) Since we consider fitting PTL models to point patterns, we can use (4.4) and (4.5) to map regularity values onto model parameters, and vice versa. If such equations are not available, figures such as Figure 3.2, Figure 3.4, Figure 3.3, Figure 4.1, Figure 4.2, Figure 4.3, and Figure 5.1 or lookup tables (not presented in this thesis) can be used for mapping as well.

Next, we give two fitting examples: one using real data of points set, and another using spatial point pattern generated by the SSI model.

Example 1 In the first example, we choose the Gaussian PTL as the targeted fitting model, and we use a real spatial point pattern extracted from the Sitefinder (see footnote 3) database. Figure 5.5(a) visualize this set of points. It consists of 61 BSs distributed over an area of $1500 \mathrm{~m} \times 1050 \mathrm{~m}$, which is centred at $\left(51.5157^{\circ} \mathrm{N}\right.$, $\left.-0.1395^{\circ} \mathrm{W}\right)$. For this pattern of points, we find that the density $\lambda=38.73 \mathrm{BSs} / \mathrm{km}^{2}$, the amount of regularity $C_{\mathrm{D}}=0.75$. For Gaussian PTL, the parameter $\tilde{\sigma}$ can be obtained by solving (4.4) for the given $C_{\mathrm{D}}$ value. We find that $\tilde{\sigma} \approx 0.3357$ for the corresponding fitted model. Figure 5.5(b) visualize a Gaussian PTL realization of the real set of points in Figure 5.5(a). (Indeed, we iteratively generate different realizations using Gaussian PTL of $\tilde{\sigma}=0.3357$ until we got the value of $C_{\mathrm{D}}$ that exactly match the regularity of the real spatial pattern. This step is not necessary if 
Table 5.1: Summary of London Local Regularity information

\begin{tabular}{|c|c|c|c|}
\hline \hline Scale of observation & Area & Density $\lambda$ & $C_{\mathrm{D}}$ \\
\hline \hline large-scale & $73.5 \mathrm{~km} \times 73.5 \mathrm{~km}$ & $0.354 \mathrm{BSs} / \mathrm{km}^{2}$ & $C_{\mathrm{D}}=1.99$ \\
\hline medium-scale & $10.5 \mathrm{~km} \times 10.5 \mathrm{~km}$ & $6.376 \mathrm{BSs} / \mathrm{km}^{2}$ & $C_{\mathrm{D}}=1.58$ \\
\hline small-scale & $1.5 \mathrm{~km} \times 1.5 \mathrm{~km}$ & $38.22 \mathrm{BSs} / \mathrm{km}^{2}$ & $C_{\mathrm{D}}=0.83$ \\
\hline \hline
\end{tabular}

we want to conduct Monte Carlo simulation since the average value of the $C_{\mathrm{D}}$ metric, for all realizations, will be the same.)

Example 2 In the second example, we use a hard-core point process to generate a simulated pattern of points. Then, we fit a uniform PTL to this simulated data. Specifically, we use SSI point process, where its density $\lambda=38.73 \mathrm{BSs} / \mathrm{km}^{2}$ and its hard-core distance $r=131.35 \mathrm{~m}$. Figure 5.6(a) illustrates a simulated set of points generated using SSI, and Figure 5.6(b) depicts an equivalent realization using uniform PTL. The amount of regularity of the spatial point pattern of Figure $5.6(\mathrm{a})$ is $C_{\mathrm{D}}=$ 0.38. This amount of regularity can be mapped to a perturbation distance $\tilde{R} \approx 0.26$ of uniform PTL using (4.4).

\subsection{The Heterogeneous Regularity of the Base Stations}

In this section, we consider the spatial structure of a set of BSs in London, UK, from three scales of observation (regions) as illustrated in Figure 5.7. They are a large-scale of observation which is a geographical region of $73.5 \mathrm{~km} \times 73.5 \mathrm{~km}$ as shown in Figure 5.7(a), medium-scale of observation which is a geographical region 


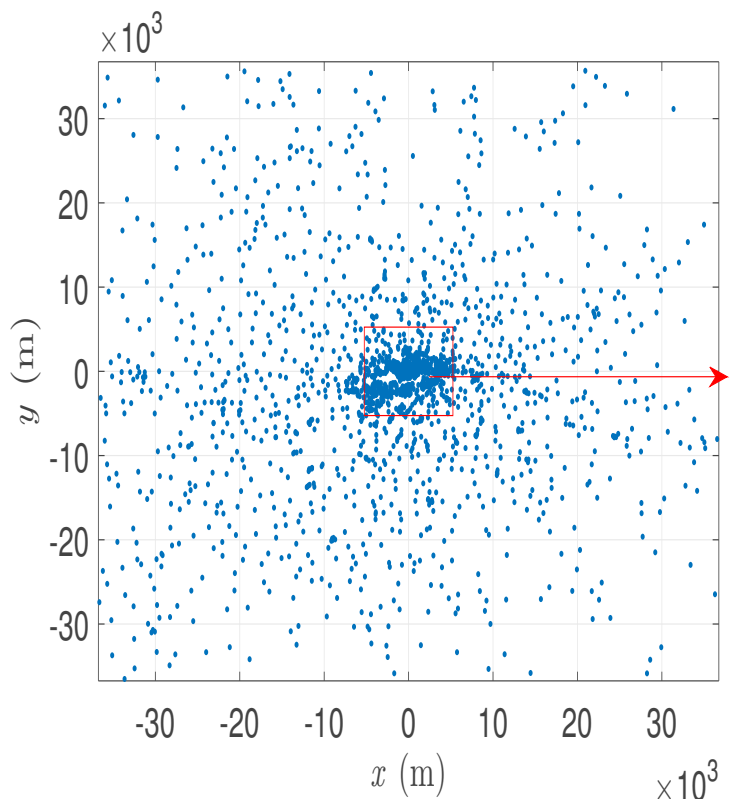

(a) Large-scale $\left(C_{\mathrm{D}}=1.99\right)$

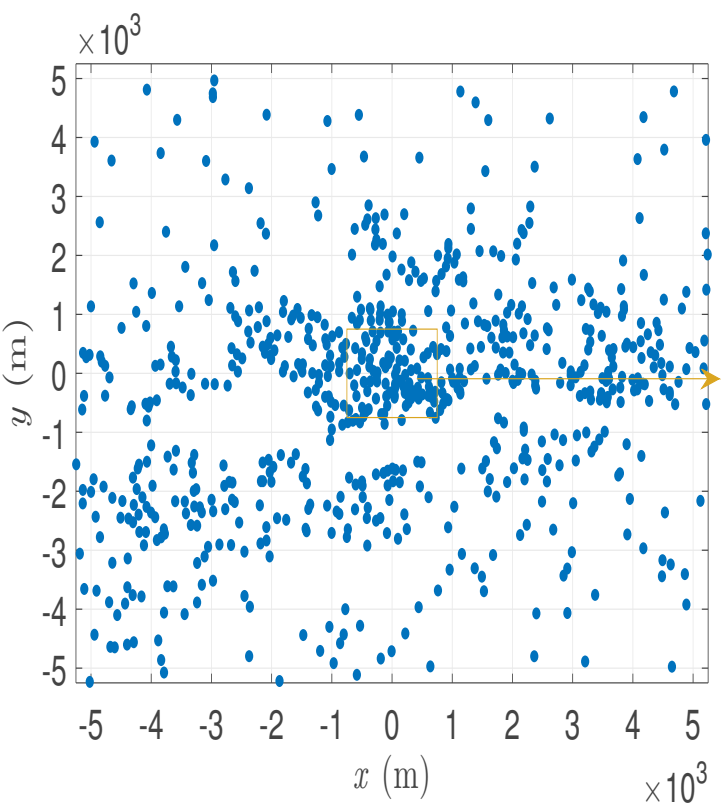

(b) Medium-scale $\left(C_{\mathrm{D}}=1.58\right)$

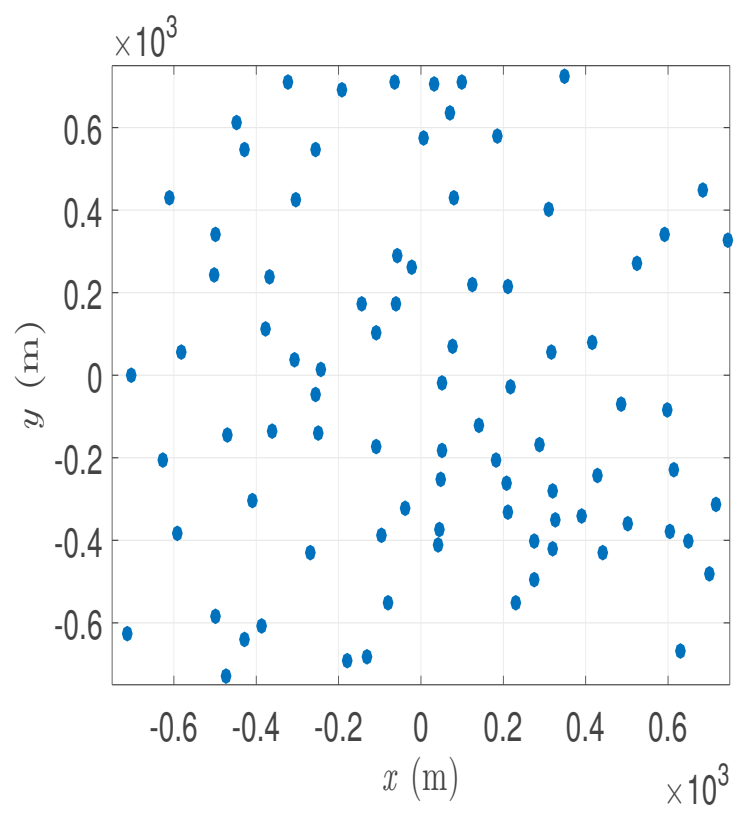

(c) Small-scale $\left(C_{\mathrm{D}}=0.83\right)$

Figure 5.7: The BSs belong to the Vodafone operator. The regions are (a) $73.5 \mathrm{~km} \times$ $73.5 \mathrm{~km}$, (b) $10.5 \mathrm{~km} \times 10.5 \mathrm{~km}$, and (c) $1.5 \mathrm{~km} \times 1.5 \mathrm{~km}$. They are centred at $\left(51.5136^{\circ} \mathrm{N}, 0.1342^{\circ} \mathrm{W}\right)$, London, UK. The small-scale region is an enlarged version of the central area (golden square) of the medium-scale region, which itself is an enlarged version of the central area (red square) of the large-scale region. 
of $10.5 \mathrm{~km} \times 10.5 \mathrm{~km}$ as shown in Figure $5.7(\mathrm{~b})$, and small-scale of observation which is a geographical region of $1.5 \mathrm{~km} \times 1.5 \mathrm{~km}$ as shown in Figure $5.7(\mathrm{c})$. The small-scale region is an enlarged version of the central area (golden square in Figure 5.7(b)) of the medium-scale region, which itself is an enlarged version of the central area (red square in Figure 5.7(a)) of the large-scale region. The centre of the observations is $\left[51.5136^{\circ} \mathrm{N}, 0.1342^{\circ} \mathrm{W}\right]$, London, UK. The BSs belong to the Vodafone operator. The data is retrieved from the Sitefinder website as aforementioned in Section 5.4.

In London, UK, the amount of the spatial relationship among the BSs changes with the scale of the observation as shown in Figure 5.7. The information of Figure 5.7 is summarized in Table 5.1. The amount of the spatial relationship of the largescale of observation, medium-scale of observation, and small-scale of observation are $C_{\mathrm{D}}=1.99, C_{\mathrm{D}}=1.58$, and $C_{\mathrm{D}}=0.83$, respectively.

While the BSs deployment is regular at a small-scale of observation, it could seem clustered as a large-scale. For example, the BSs at a city scale exhibit repulsion among each other, but at a country/state scale their spatial distribution may appear clustered, especially in the sparsely populated countries. In other words, the deployment of the BSs of a large-scale geographical region can be partitioned into different smaller areas of locally regular structure although the large-scale (global) structure of these BSs may look like clustered. We call this observation the heterogeneous regularity of the BSs. Figure 5.7 also shows that the density of the BSs in London is not a constant at a large-scale of observation. The density of the BSs increases as we move from the suburban to the dense urban areas. (This variation in the density of the BSs is most likely because of the variation of population's density since there is a strong correlation between population and the BSs densities [49].) This change in density of the BSs makes them appear clustered in large-scale scenarios and causes the variation in the amount of the spatial regularity. At a small-scale of observation, 
the local structure of the BSs is regular (repulsive) because the BS density is almost constant. The spatial structure of the BSs deployment tends to change with the scale of observation if their density is not constant.

Based on the aforementioned observations, it is invalid to model the BSs in largescale scenarios with the existing clustered point processes since the locations of the points in their clusters are completely random rather than being repulsive. It is more appropriate to have new models of clusters of repulsive points to maintain the local regularity of the BSs deployment. To this end, a combination of repulsive spatial patterns of different densities and amount of spatial regularity can be used to model such a large-scale scenario. Finding such models is beyond the scope of this research. In this thesis, our focus is only on local structures where the average density of the macro BSs in the deployment remains constant.

The concept of the heterogeneous regularity is applicable to the small cells (e.g., micro-, pico-, and femtocells) deployments as well, but maybe with a different definition of the scale of observation. Like the deployment of the macro cells, small cells have repulsive local structure and may appear clustered in the large-scale scenario. For example, small cells overlaid by a particular macro BS have local regularity, but they may appear clustered on a city scale.

\subsection{Concluding Remarks}

- We tested three geometry-based metrics for quantifying the spatial relationship of a set of points and for capturing the network performance. We showed that the $C_{\mathrm{D}}$ metric is the most accurate indicator for the network performance: a difference in the regularity can be directly interpreted as a difference in the network performance. 
- We proposed a novel approach to approximate the performance of the repulsive networks compared to a reference model based on the difference in the amount of regularity.

- We devised a new approach for fitting point processes to the spatial deployment of BSs using $C_{\mathrm{D}}$ metric.

- We showed that the actual deployment of the BSs is locally regular. However, however, at a larger scale, the BSs may appear clustered around towns. Further research is necessary to find more realistic models for this phenomenon.

- In this chapter, we modelled the location of the BSs using PTLs and mapped other models to them because of their simple and efficient implementation, their full regularity range (from the TL to the PPP), and their prevalence in the industry and in recent wireless literature. This chapter supports the conclusion of Chapter 4, which is the PTL is our model of choice. 


\section{Practical Applications}




\section{Chapter 6}

\section{Cell Switch-Off for Networks Deployed With Variable Spatial Regularity}

"If you define the problem correctly, you almost have the solution."

—Steve Jobs

\subsection{Introduction}

Wireless cellular networks have seen an unprecedented growth in data traffic demand. This demand requires an increase in the number of base stations (BSs) in order to alleviate the network's capacity shortage during the peak traffic. While many of these BSs are underutilized during low traffic periods, BSs are the most significant consumers of cellular network power. BSs are responsible for $57 \%$ of power consumption in cellular networks, yet this energy consumption of these BSs is almost independent of their traffic load [50-53]. Each BS absorbs an average of 25 MWh per year, which may approximately cost $\$ 3,000$ per year [52]. Clearly, energy consumption raises the concern of cellular network operators regarding operational expenditure (OPEX). Another aspect is the impact of the carbon footprint associated with cellular networks on the environment. This urges the researchers to innovate new techniques to make the cellular networks more energy-efficient. Turning off some BSs 
while maintaining good service quality during low-traffic periods is an opportunistic energy-efficient approach $[54,55]$. It would save a remarkable amount of energy, and hence reduce both the network's OPEX and greenhouse gas emissions [56]. The energy saved is proportional to the number of the switched-off BSs. This approach is called cell switch-off (CSO) [56], which is an important topic under the umbrella of green communications $[50-53,56]$.

In the current CSO literature, most authors consider the TL (i.e., the hexagonal layout): the most regular arrangement for BSs deployment. Recently, a few works such as $[57,58]$ have modelled the BS locations using the PPP. The PTLs span the entire range between the TL and the PPP. Therefore, we employ a PTL for modelling the BS locations in this chapter. ${ }^{1}$ To the best of our knowledge, The RPPs have not been used to model the BS locations in the CSO context.

\subsubsection{Motivation}

In Chapter 3 and Chapter 4, we show that "the regularity maximizes the coverage probability" [24]. This is confirmed in [23] and [13] for cellular networks by showing that the best signal-to-interference ratio (SIR) distribution is achieved when BSs are placed on the perfect regular TL; the worst is found when the BSs are deployed according to a PPP. The higher the regularity of the BS deployment, the better the coverage probability and SIR distribution. Ideally, the network operators would maintain the coverage requirement or even improve it while saving the energy and its expenditure during low-traffic demand by switching off some BSs. In practise, they may only want to avoid a drastic drop in network coverage. Regardless of the operator's intention, the intuitive approach to attaining the best possible coverage quality is to maximize the regularity of the remaining active BSs. Although there

\footnotetext{
${ }^{1}$ This chapter has been published in the IEEE Wireless Communication Letters [59].
} 
is no explicit research stream focusing on regularity maximization in CSO, this has been done in some previous CSO literature. Some works that use hexagonal layout propose regular static CSO patterns that maintain regularity and provide best possible coverage probability - this is the case in [60], for example. As for a PPP-deployed network, Cho and Choi [57] propose a "repulsive cell activation strategy" that thins out (switches off) some of the BSs, resulting in a hard-core RPP.

\subsubsection{Contribution}

Our main contribution in this chapter is that we identify and study a novel CSO problem that has not been previously addressed in the literature. Starting with BSs deployed with a variable amount of spatial regularity, we focus on maximizing the network's performance through maximizing the spatial deployment regularity of the remaining active BSs. As a starting point to solve this problem, we propose simple and intuitive, yet practical, algorithms that perform better than random switch off, particularly for highly irregular BS deployments. The performance of these algorithms is compared to upper and lower bounds. They are much better than random CSO, particularly for highly irregular BS deployments. Finally, we apply one of these algorithms to a real set of BSs.

The rest of the chapter is organized as follows: In Section 6.2, the research problem is defined. In Section 6.3, simple CSO algorithms are proposed. In Section 6.4, we defined the system setup. We evaluate the performance of the algorithms in Section 6.5. Finally, we draw conclusions in Section 6.6 


\subsection{CSO as a Regularity Maximization Problem}

Given a set of BSs deployed using a repulsive spatial model, we consider switching off some of them in order to save energy during low-traffic periods while maximizing the network performance of the remaining active BSs. An important wireless network performance metric worth maximizing is the coverage probability, which depends on the SIR distribution. The network performance can be maximized by increasing the spatial regularity of the active BSs.

In the preceding chapters, we have mathematically quantified the regularity of a spatial layout of $\mathrm{BSs}$ in a rigorous way using $\mathrm{CoV}$-based scalar metric(s). Among these metrics, we choose the $\mathrm{CoV}$ of the lengths of Delaunay triangulation edges $\left(C_{\mathrm{D}}\right)$ to measure the change in regularity resulting from applying the CSO algorithms. As shown in the preceding chapters, as $C_{\mathrm{D}}$ decreases, the spatial regularity increases (i.e., irregularity decreases). That means minimizing $C_{\mathrm{D}}$ leads to maximizing the regularity. The metric $C_{\mathrm{D}}$ is presented in detail in Chapter 2 .

\subsubsection{The Problem Statement in Brief}

Given a set $\mathcal{A}$ of $N$ BSs, which can be modelled using an RPP, we aim to switch off $L$ BSs and leave the remaining $M=N-L$ BSs active such that their regularity is maximized, thus also maximizing the coverage probability. Our objective is to select the active BSs to be as far apart from one another as possible. The CSO percentage is

$$
\rho=\left(\frac{L}{N}\right) \times 100 \%
$$

One way to ensure the active BSs are spread out as far as possible is to maximize the nearest neighbour distance for each BS. This is called the $p$-dispersion problem [61], which is usually formulated as mixed-integer linear programming and has been known 
to be NP-hard [61]. Kuby's [62] formulation for the $p$-dispersion problem can be written as

$$
\begin{array}{ll}
\operatorname{maximize} & r, \\
\text { subject to } & \sum_{j \in \mathcal{A}} z_{j}=M, \\
& r \leq d_{i j}\left(1+D\left(1-z_{i}\right)+D\left(1-z_{j}\right)\right), \quad \forall i, j \in \mathcal{A}, i<j, \\
& z_{j} \in\{0,1\} \quad \forall j \in \mathcal{A} .
\end{array}
$$

Here, $r=\min _{i<j}\left\{d_{i, j}\right\}$ is the smallest separation distance between any pair of active BSs, where $d_{i, j}=\left\|x_{i}-x_{j}\right\|$ is the distance between a BS $i$ and $j \in \mathcal{A}$. (For simplicity, we refer to the BSs and their locations by the indexes.) The Boolean variable $z_{j}$ indicates whether a BS at location $j$ is switched off or not. $M$ is the number of the required remaining active $\mathrm{BSs}$, and $D$ is a very large constant, e.g., $\max _{i<j}\left\{d_{i, j}\right\}$. Constraint $6.2 \mathrm{~b}$ requires $M$ BSs to be active. Constraint 6.2c holds (imposes an upper bound on the minimum separation distance) only if BSs are active at both location $i$ and $j$. Next, we suggest several heuristic methods to deal with this problem.

\subsection{Proposed Algorithms for Cell Switch-Off}

Due to the difficulty of solving this problem optimally, we propose three greedy heuristic algorithms to tackle it. Greedy algorithms are very simple and intuitive. They make a locally optimal (greedy) iterative decision and have two native varieties: greedy construction and greedy deletion. While the greedy construction is initialized by an empty solution set, then points are added to the solution, the greedy deletion is begun with all candidate points as a solution, then points are removed at each 
iteration.

The following notations are used to describe these algorithms. While $\operatorname{dist}\left(x_{i}, x_{j}\right)$ denotes the euclidean distance between the points $x_{i}$ and $x_{j}, \operatorname{dist}\left(x_{j}, \mathcal{B}\right)$ denotes the distance between the point $x_{j}$ and its nearest neighbor point in the set $\mathcal{B}$. The number of points in the set of points $\mathcal{B}$ is written $|\mathcal{B}|$. The input of the algorithms is the set of BSs locations $\mathcal{A}=\left\{x_{1}, x_{2}, \ldots, x_{N}\right\}$ and the desired number of active BSs $M$; the output is a set $\mathcal{B}=\left\{y_{1}, y_{2}, \ldots, y_{M}\right\}$ of active BSs with maximized regularity.

\subsubsection{Greedy Construction (GC)}

The solution set of the GC is initialized by selecting the two furthest points. Then, at each iteration, a new point, the one farthest apart from all points in this solution set, is added until the targeted number of active points $M$ is accumulated in the solution set [61]. Nodes that are not in the solution set are to be switched off. Algorithm 1 illustrates the pseudo-code of the GC algorithm [61]. The complexity of $\mathrm{GC}$ in terms of distance computation is at most $O\left(N^{3}\right)$.

\footnotetext{
Algorithm 1: Greedy construction (GC).

Input : The set of BSs locations $\mathcal{A}=\left\{x_{1}, x_{2}, \ldots, x_{N}\right\}$ and the desired number of active BSs $M$.

Output : A set $\mathcal{B}=\left\{y_{1}, y_{2}, \ldots, y_{M}\right\}$ of active BSs with maximized regularity.

1 Find $x_{v}, x_{w} \in \mathcal{A}$, such that $\operatorname{dist}\left(x_{v}, x_{w}\right)=\max \left\{\operatorname{dist}\left(x_{i}, x_{j}\right): x_{i}, x_{j} \in \mathcal{A}, \forall i, j\right\}$

$2 \mathcal{B} \Leftarrow\left\{x_{v}, x_{w}\right\} ; \quad \mathcal{A} \Leftarrow \mathcal{A} \backslash\left\{x_{v}, x_{w}\right\}$

3 while $|\mathcal{B}|<M$ do

$4 \quad$ Find $x_{z} \in \mathcal{A}$, such that $\operatorname{dist}\left(x_{z}, \mathcal{B}\right)=\max \left\{\operatorname{dist}\left(x_{j}, \mathcal{B}\right): x_{j} \in \mathcal{A}\right\}$

$5 \quad \mathcal{B} \Leftarrow \mathcal{B} \cup\left\{x_{z}\right\} ; \quad \mathcal{A} \Leftarrow \mathcal{A} \backslash\left\{x_{z}\right\}$

6 end

7 return $\mathcal{B}$
} 


\subsubsection{Greedy Deletion (GD)}

The GD is initialized by identifying the two closest BSs in the current solution set and switching off the one that has the smallest distance to its second-nearest neighbour. This iteration is repeated until the specified number of BSs is turned off [61]. A pseudo-code is described in Algorithm 2. The complexity of GD is at most $\mathcal{O}\left(N^{3}\right)$.

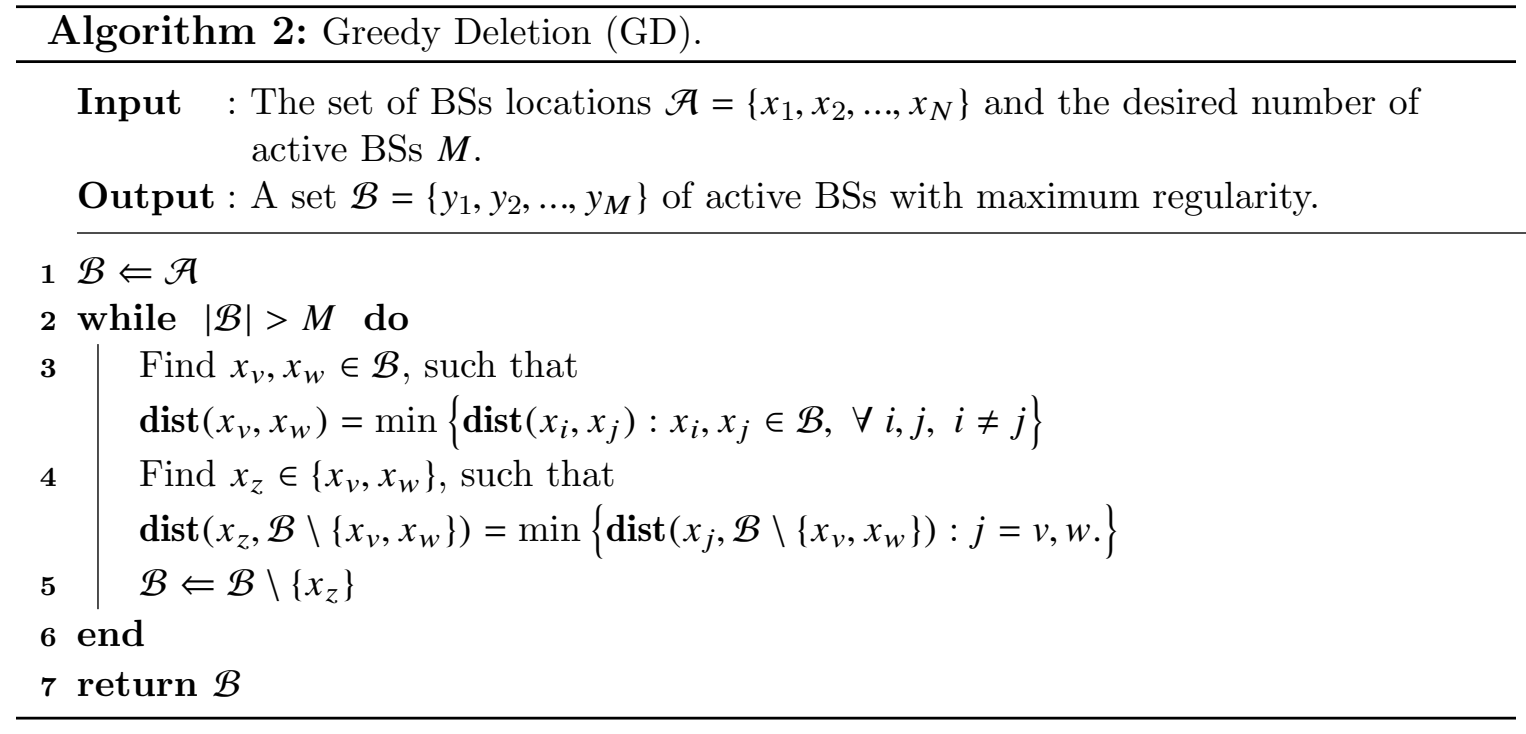

\subsubsection{Semi-Greedy Deletion (SG)}

This is very similar to the GD algorithm. The only difference is that it employs random selection for removal between the two closest points [61]. That is to say, the point $x_{z} \in\left\{x_{v}, x_{w}\right\}$ in Algorithm 2 is chosen randomly.

\subsubsection{Random Switch-Off (RS)}

BSs are randomly switched off without any rules apart from the number of BSs required to be turned off [58]. 
Moreover, separate PPP-deployed and TL-deployed networks - that have the same density as the remaining active BSs of the partially switched-off network - are used as a loose lower bound and a non-achievable upper bound, respectively.

\subsection{Downlink System Model}

\subsubsection{BSs Deployment}

We consider a network layout modelled using the perturbed triangular lattice (PTL) $[13,41]$, an RPP with variable regularity. The advantage of the PTL model is that it covers the whole regularity range between the TL and the PPP [41], unlike many other RPPs [23]. Generating the PTL starts with generating TL, and then independently displacing each point (BS location) by a random independent vector. We consider uniform displacement on a disk which leads to uniform PTL. The radius $R$ of the disk controls the amount of regularity. It is normalized by the inter-site distance $\eta$ of the TL as $\tilde{R}=R \eta^{-1}$. The metric $C_{\mathrm{D}}$ is then a function of $\tilde{R}$. The density of the TL and also the PLT is $\lambda=\frac{2}{\sqrt{3}} \eta^{-2}$. Details are presented in Chapter 4.

The BSs serve with full buffer. They transmit at the same fixed power level and have the same operating frequency. They have one sector and one omni-directional antenna.

\subsubsection{Users Model}

Independent of the network deployment, the users are modelled as a PPP. Each user is tagged to the BS of the strongest signal power. Users have best effort service with equal resource allocation. They receive Rayleigh faded signals. 


\subsubsection{LTE Urban Macro (UMa) Scenario}

Channel parameters and all other specifications follow the UMa scenario presented in [63]. We assume the proportional fair scheduling scheme.

\subsubsection{Simple Scenario}

We only consider this simple scenario for calculating SIR gain to make some of the results comparable with [45]. It has simple path loss model with a path loss exponent of 4 and no shadowing. The thermal noise is ignored.

\subsection{Results}

We consider a network with 665 BSs deployed over an area of $12 \mathrm{~km} \times 12 \mathrm{~km} .10$ users per BS are distributed over the whole area, but we only consider users within the central area of $7.2 \mathrm{~km} \times 7.2 \mathrm{~km}$ in order to eliminate the edge effects. We sweep the BS regularity from TL $\left(C_{\mathrm{D}}=0\right)$ to PPP (asymptotically, $C_{\mathrm{D}} \rightarrow 1$ ) using the tunable PTL. We apply the CSO algorithms presented in Section 6.3 on these BS deployments. With $5 \%$ steps, we switch off up to $90 \%$ of the BSs. The saved energy is proportional to the number of turned-off BSs [60]. For brevity, only specific CSO percentage $\rho$ values are reported in Figures 6.1-6.5.

Figure 6.1 shows the change in the network regularity before $\left(C_{D}(\right.$ in $\left.)\right)$ and after $\left(C_{\mathrm{D}}\right.$ (out) $)$ applying the CSO algorithms. The lower the $C_{\mathrm{D}}$ value, the higher the regularity of BSs. For BSs deployed with very high regularity, the proposed CSO algorithms deteriorate their regularity. However, this may still be close to the best achievable regularity if some BSs are switched off. The greedy algorithms improve the spatial regularity when the BSs are deployed with low regularity $\left(C_{\mathrm{D}} \gtrsim 0.5\right)$. The line $C_{\mathrm{D}}$ (out) $=\mathrm{C}_{\mathrm{D}}($ in) separates the graphs in Figure 6.1 into two regions: above the line, 
where the regularity is deteriorated, and below, where the regularity is improved.

\subsubsection{SIR Gain}

Taking a PPP-deployed network as a reference, we use the horizontal gap (SIR gain) between the SIR distributions of the PPP- and the PTL-deployed networks as the performance metric; specifically, the SIR gain at target probability 50 percent: $G_{\mathrm{p}}(0.5)[13,45,46]$. This gain is also called the deployment gain [13]. Figure 6.2 shows the comparison between different CSO algorithms at different $\rho$ values in terms of the SIR gain for BSs deployed with variable amounts of regularity.

Figure 6.1 shows that as the degree of freedom increases for high $\rho$ values, the algorithms significantly enhance the regularity (e.g., reducing $C_{\mathrm{D}}$ to around 0.4 for GC). The SIR gain is hence improved in Figure 6.2. Being completely regular, the TL-deployed network has an SIR gain of $3.4 \mathrm{~dB}$, the highest SIR gain compared to a PPP-deployed with simple scenario assumptions in Section 6.4.4. This $3.4 \mathrm{~dB}$ is consistent with the literature [45]. In terms of regularity and SIR gain, the SG algorithm does not perform as well. While the GD algorithm performs better for low CSO percentage $(\rho<55 \%)$, GC dominates for higher $\rho$ values. An interesting observation is that even when GD performs better, GC still performs comparably well, within a $0.1 \mathrm{~dB}$ SIR difference. Thus, if an operator prefer to select only one algorithm for the entire switch-off scale, we recommend GC.

In the extension of this work [64], we tested other heuristic algorithms. We found that GC performs very well regardless of the percentage of the switched-off BSs and the spatial regularity of the BS locations.

Note that GD and SG usually perform poorly when they are applied on the TL, since all points are equally spaced. Thus, we exclude from this work applying the 


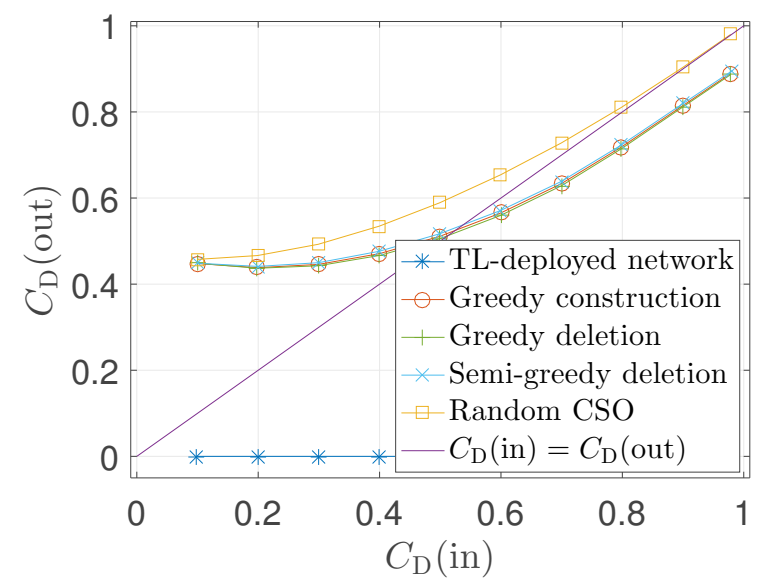

(a) $\rho=10 \%$

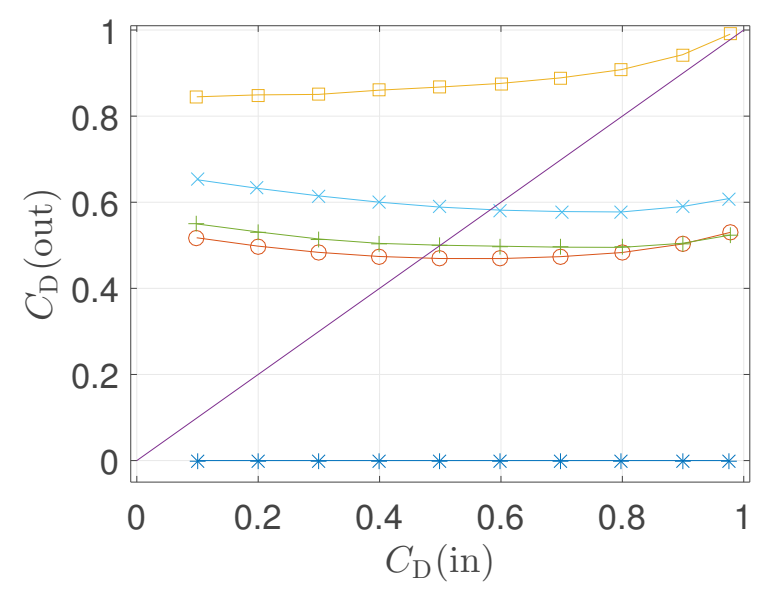

(c) $\rho=65 \%$

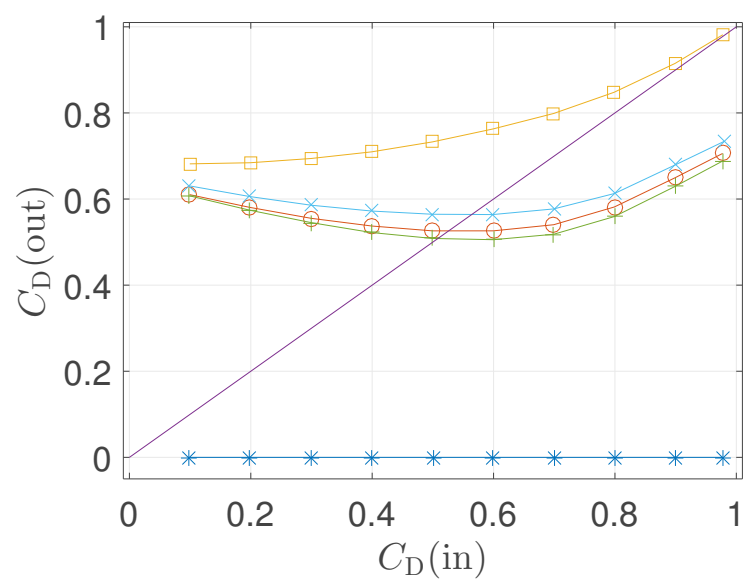

(b) $\rho=35 \%$

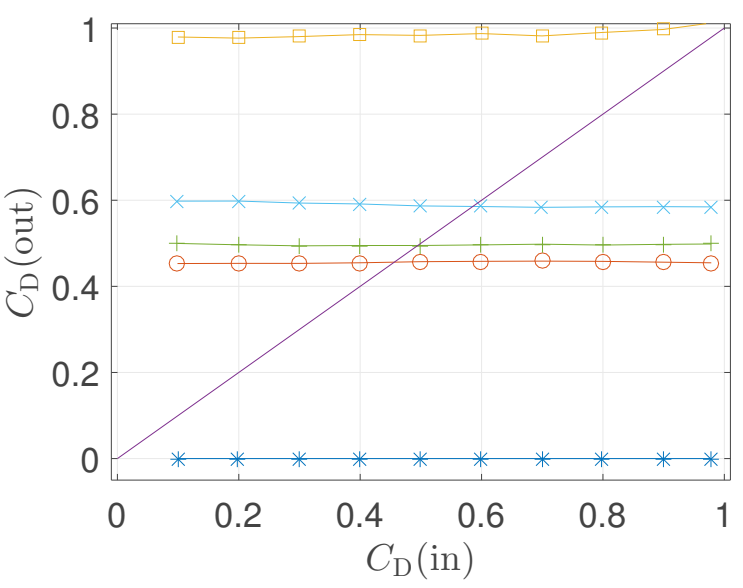

(d) $\rho=90 \%$

Figure 6.1: Network deployment regularity before $\left(\boldsymbol{C}_{\mathrm{D}}(\mathrm{in})\right)$ and after $\left(\boldsymbol{C}_{\mathrm{D}}(\mathrm{out})\right)$ applying the CSO algorithms at different CSO percentages $\rho$. 


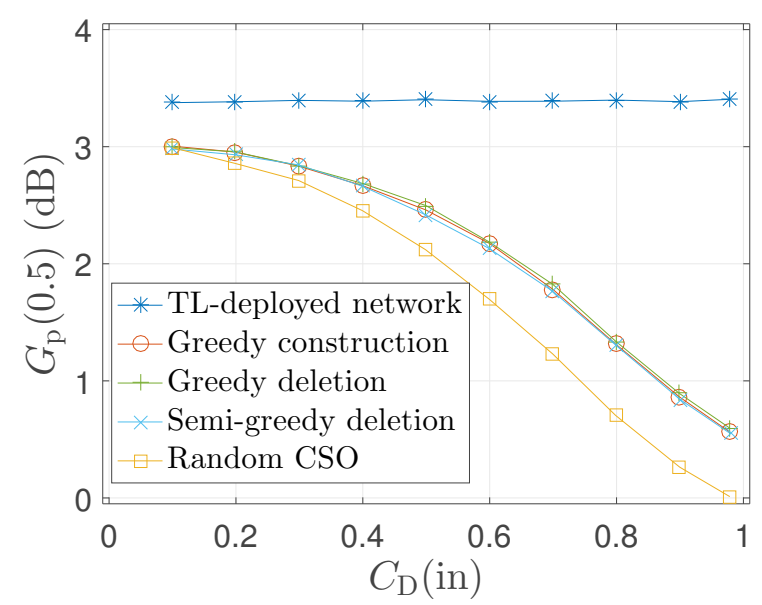

(a) $\rho=10 \%$

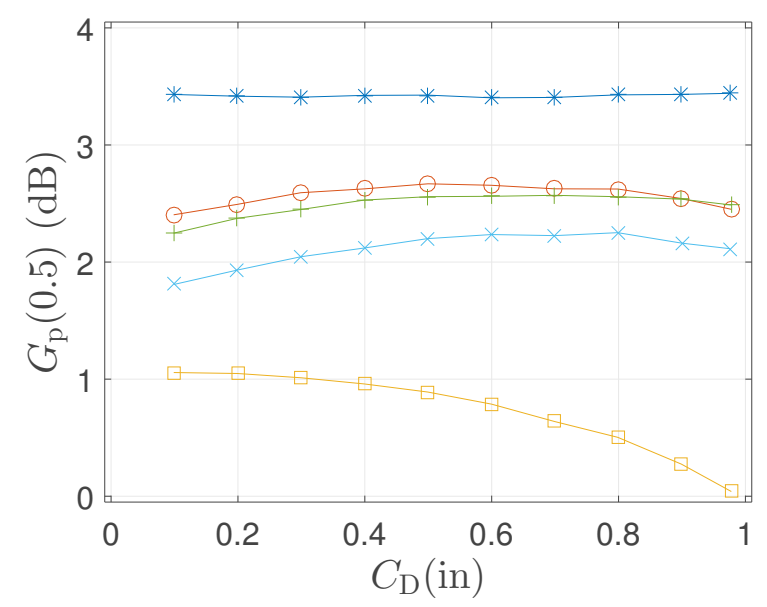

(c) $\rho=65 \%$

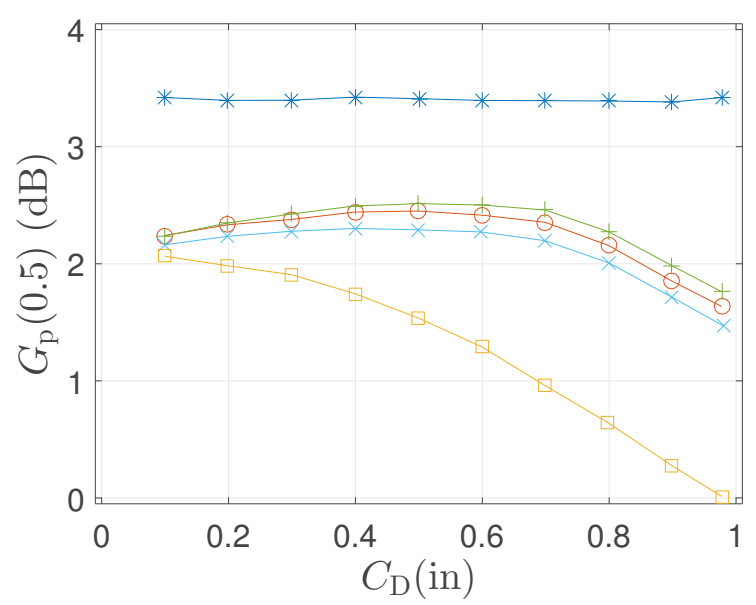

(b) $\rho=35 \%$

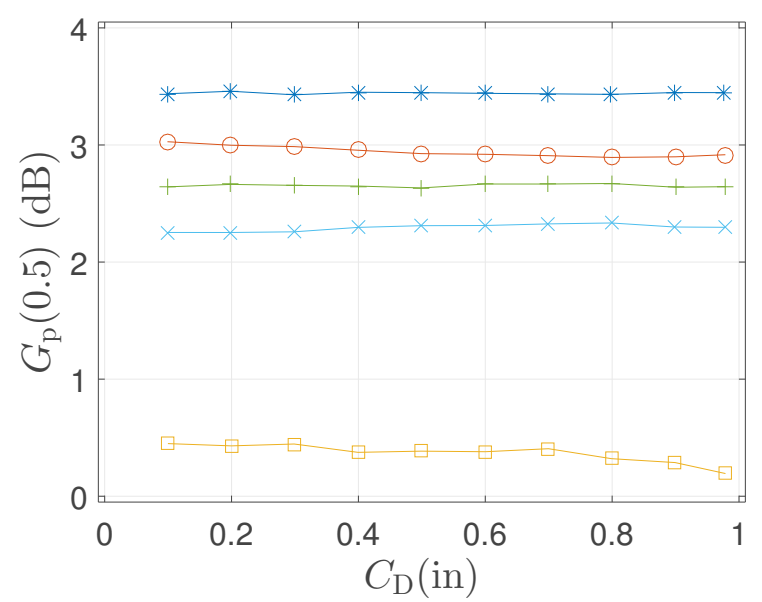

(d) $\rho=90 \%$

Figure 6.2: The SIR gain $G_{\mathrm{p}}(0.5)$ as a function of the network deployment regularity before the $\operatorname{CSO}\left(C_{\mathrm{D}}(\mathrm{in})\right)$ at different CSO percentages $\rho$. 
CSO algorithms on the idealized TL deployment.

\subsubsection{Network Capacity}

We define the network capacity as the sum of rates of all users in the system in bits/Hz. We normalize the capacity of each deployment by its full capacity before the switch-off. The normalized capacity as a function of the CSO percentage for different CSO algorithms is shown in Figure 7.5. The number of the switched-off BSs is a function of the decrease in the network's data traffic demand. For a network deployed according to a PPP (see Figure 6.3(b)), if the demand drops by $20 \%$, the operator could switch off up to $37 \%$ of the BSs using either greedy algorithm, whilst only $20 \%$ of the BSs could be switch off using random CSO.

\subsubsection{Rate Coverage}

Defining the rate coverage as the probability that a randomly located user achieves a rate greater than the rate threshold $\mathcal{R}_{\text {th }}$, the coverage probability for $\mathcal{R}_{\text {th }}=512$ kbps for two BS deployments as a function of the CSO with applying different CSO algorithms is shown in Figure 6.4. With spatial regularity maximizing algorithms, users achieve higher rates compared to random CSO.

\subsubsection{Real BS Locations}

As an example, we apply GD on a real deployment of BSs, taken from Ofcom ${ }^{2}$ and shown in Figure 6.5. As seen in Figure 6.5, when we use GD, as more BSs are

\footnotetext{
${ }^{2}$ Independent regulator and competition authority for the communications industries of the UK. Ofcom website: https://www.ofcom.org.uk/
} 


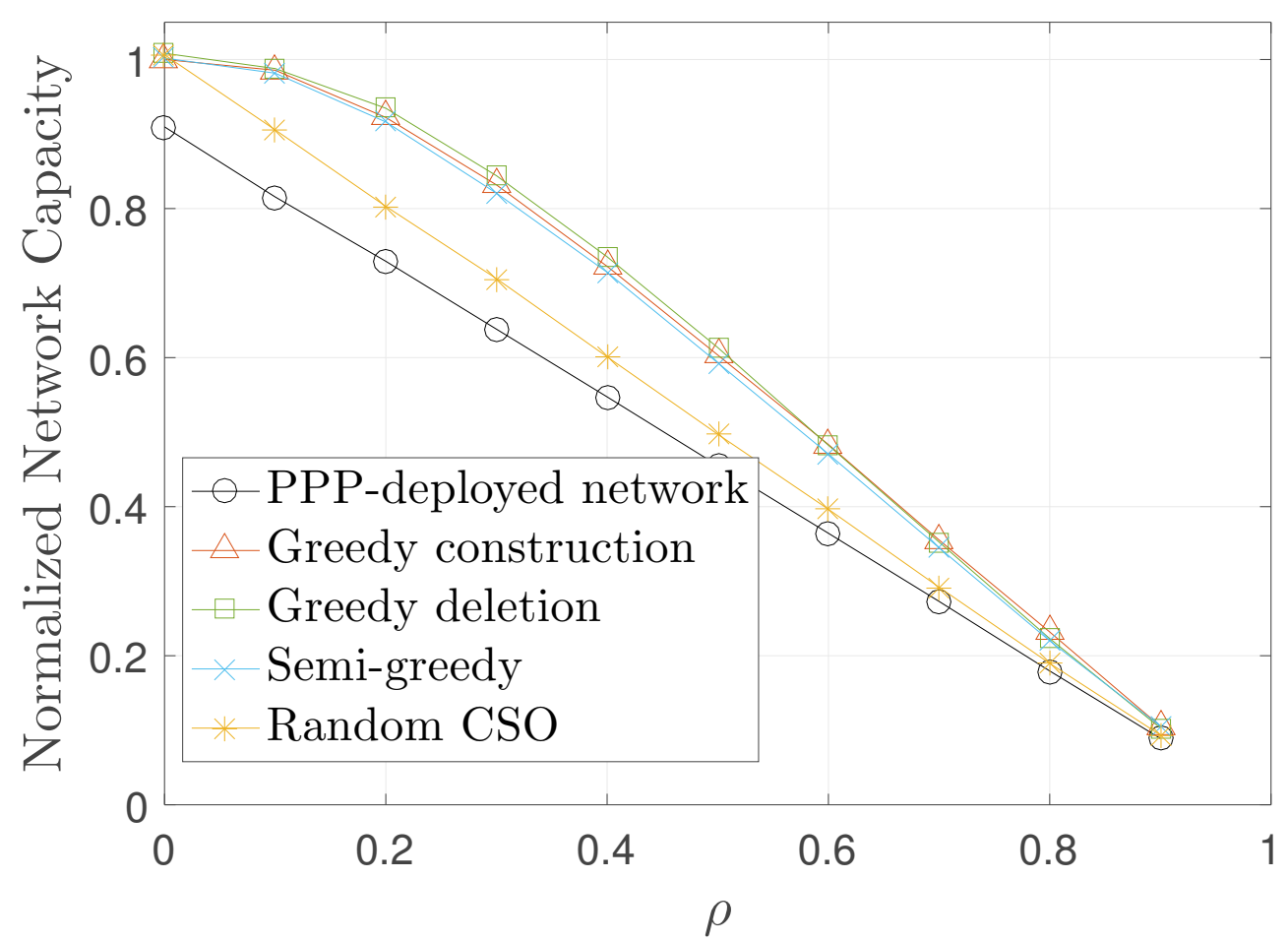

(a) $C_{\mathrm{D}}($ in) $=0.8$

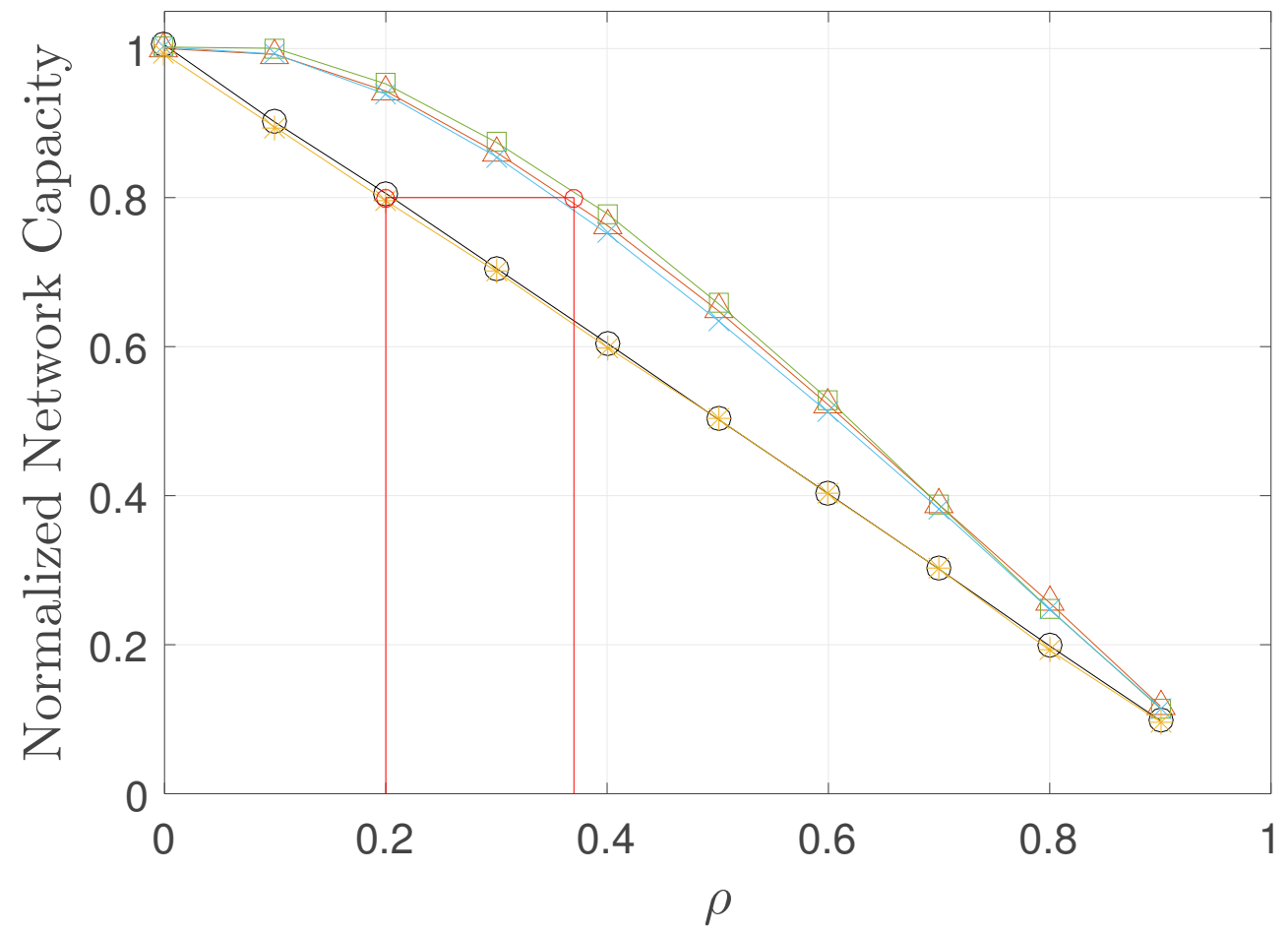

(b) $C_{\mathrm{D}}(\mathrm{in})=1(\mathrm{PPP})$

Figure 6.3: Normalized network capacity for LTE UMa scenario as a function of the CSO percentages $\rho$ for two different network deployment in terms of regularity. The regularity maximization gain is annotated by thick red line (b). 


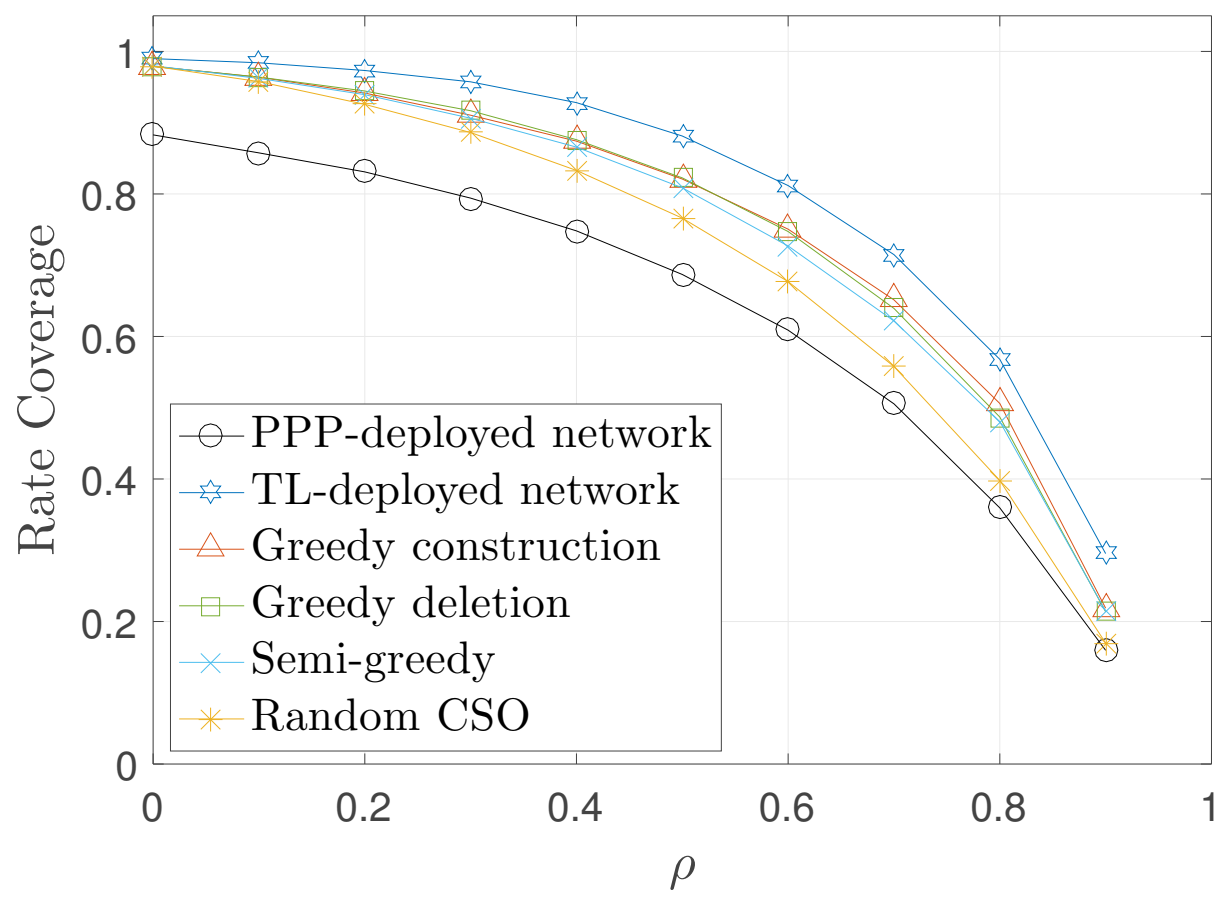

(a) $C_{\mathrm{D}}($ in) $=0.4$

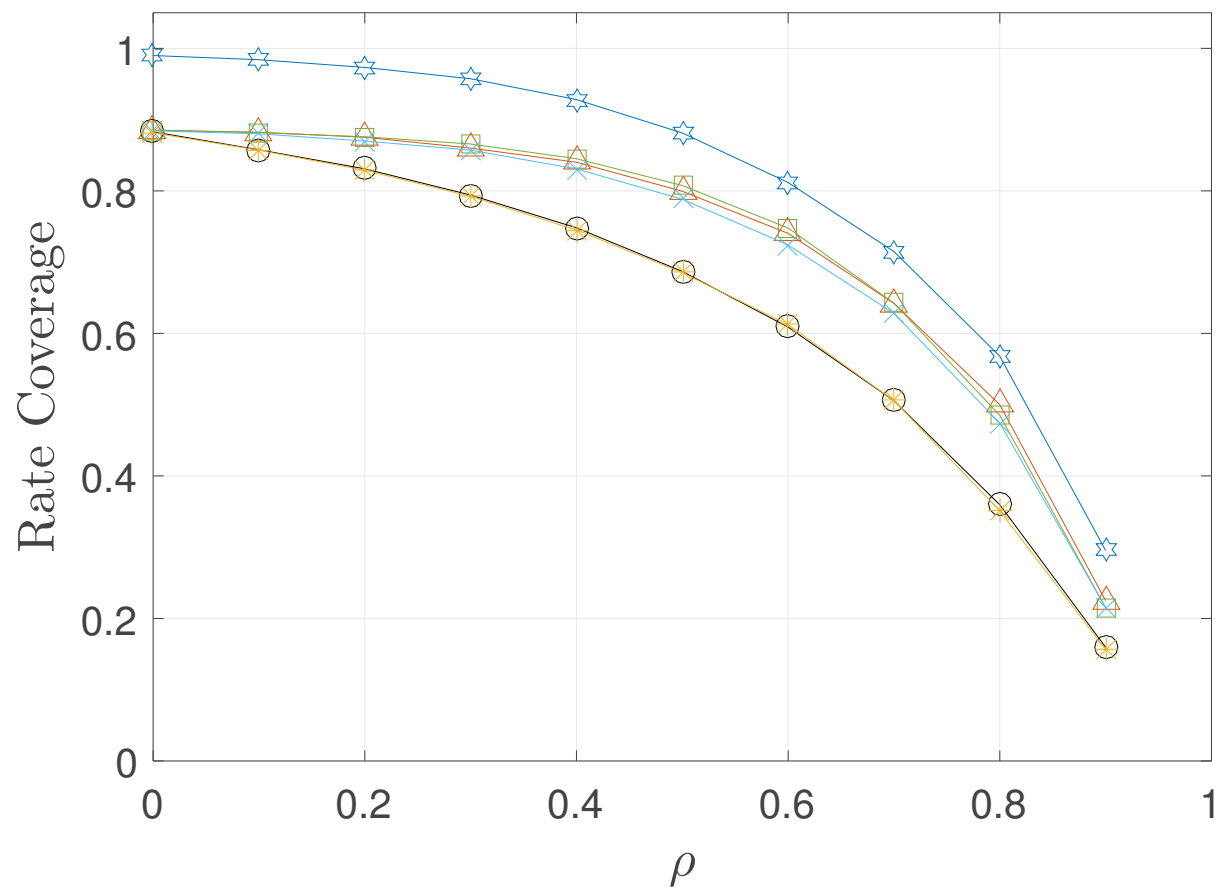

(b) $C_{\mathrm{D}}(\mathrm{in})=1(\mathrm{PPP})$

Figure 6.4: Rate coverage for LTE UMa scenario as a function of the CSO percentages $\rho$ for two different network deployment in terms of regularity. 
switched off, the previously turned-off BSs are never turned back on. On the other hand, Figure 6.6 shows that the SG has no consistent switch-off pattern.

The advantage of GC and GD is that the current solution is a subset of the previous solution if $\rho$ is increased and a superset if $\rho$ is decreased. That is, the first switched-off BS is the last to be turned on when the $\rho$ changes. This is a very practical feature for cellular network operators since it reduces the on-off/off-on transitions. The disadvantage is that the accuracy of the solution may decrease for GC if the targeted number of the BSs for switching-off is low and for GD if it is high.

\subsection{Concluding Remarks}

- This work expands the CSO literature by modelling BSs using RPPs.

- We shed light on a new interesting CSO problem that relates the network's performance improvement to the maximization of the spatial regularity of the active cells.

- We proposed simple greedy algorithms to solve it. They are particularly effective for BSs deployed with low regularity. The SG algorithm has inferior performance.

- Depending on the CSO percentage, the GC algorithm provides comparably good or superior performance compared to the others. Therefore, if we want to implement only one algorithm, we can safely implement the GC algorithm. GD and GC algorithms are practical since the CSO always follows the same switching pattern.

- We also applied the GD on a real BS deployment. 


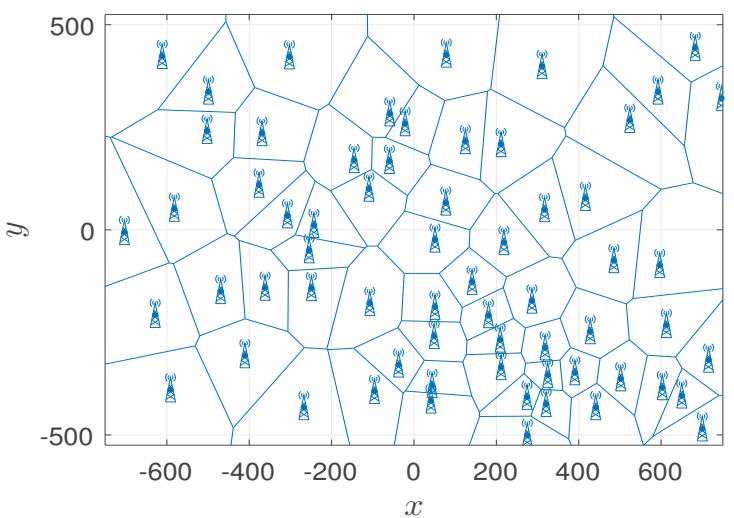

(a) $\rho=0 \%, C_{\mathrm{D}}=0.806$

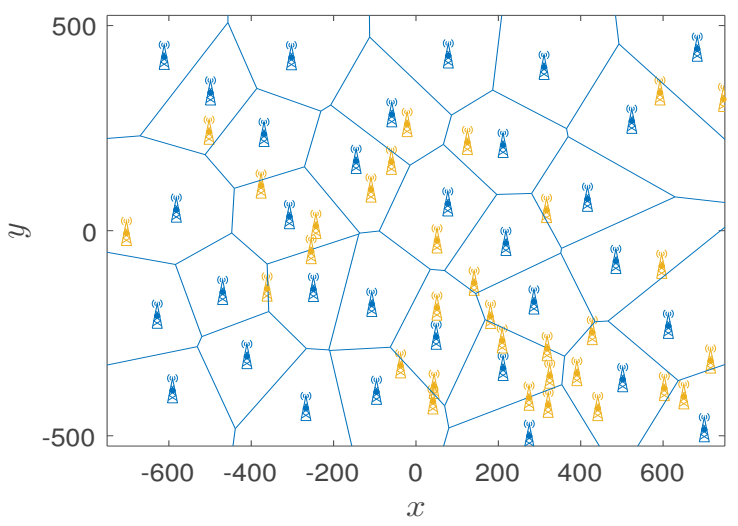

(c) $\rho=50 \%, C_{\mathrm{D}}=0.46$

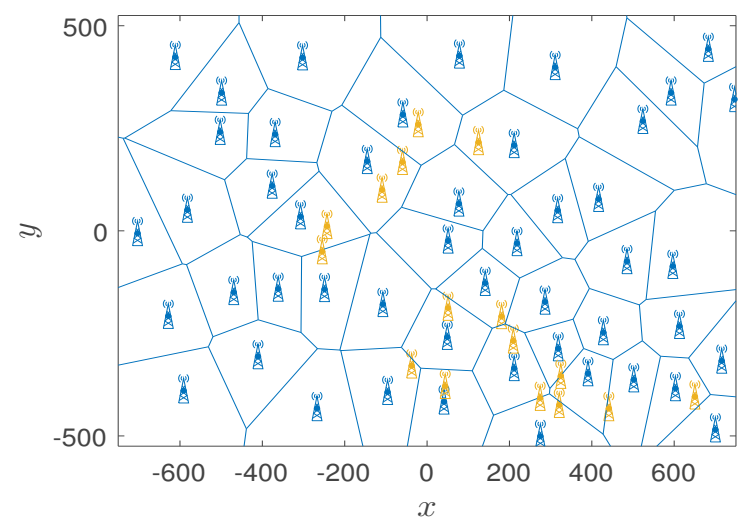

(b) $\rho=25 \%, C_{\mathrm{D}}=0.61$

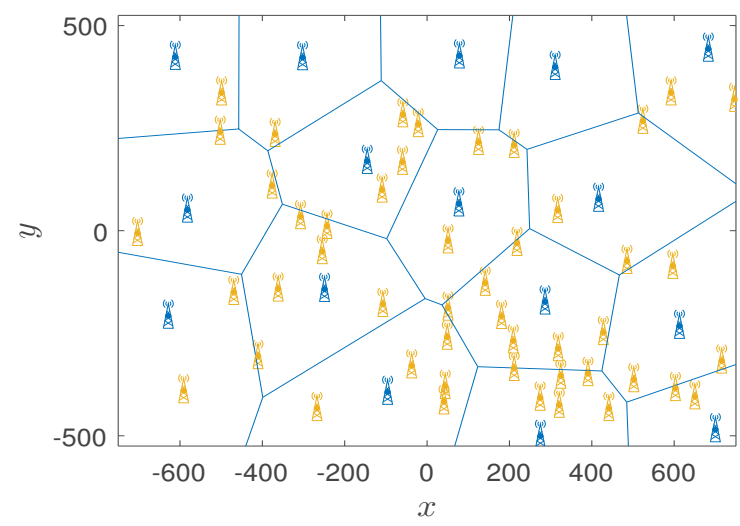

(d) $\rho=75 \%, C_{\mathrm{D}}=0.35$

Figure 6.5: Applying the greedy deletion (GD) algorithm on real BS locations at different CSO percentages $\rho$. Blue and golden towers represent the active and inactive BSs, respectively. The BSs (GSM band $900 \mathrm{MHz}$ ) belong to the Vodafone operator. The region is $1500 \mathrm{~m} \times 1050 \mathrm{~m}$, in London, UK, centred at $\left(51.5136^{\circ} \mathrm{N}, 0.1342^{\circ} \mathrm{W}\right)$. This data is similar to that used in [13, Fig. 1].

- With spatial regularity maximization CSO, either better network performance is achieved or extra BSs could be turned off (i.e., saving more energy) while maintaining the same quality-of-service (satisfying network capacity demand and/or rate coverage) compared to random CSO.

- The regularity maximization gain is significant for highly irregular BS deployments.

- This work is extended for testing other heuristics in [64]. 


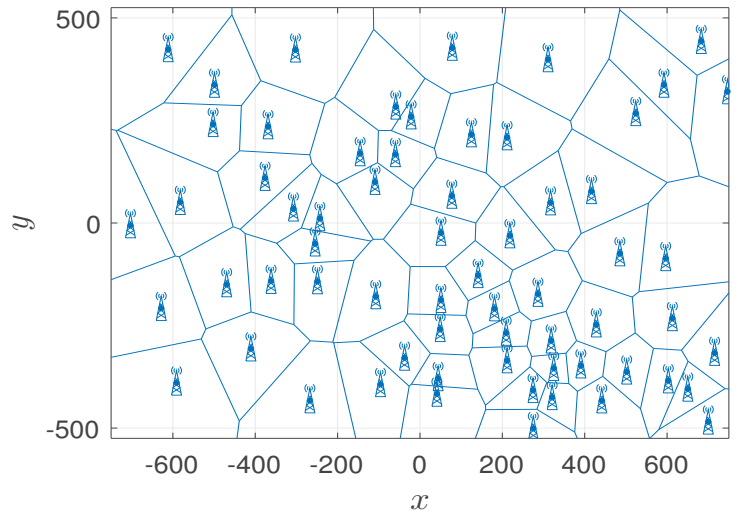

(a) $\rho=0 \%, C_{\mathrm{D}}=0.806$

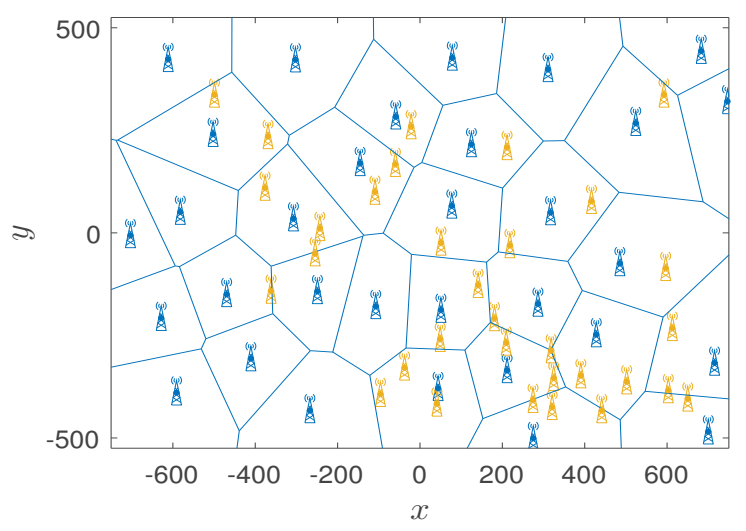

(c) $\rho=50 \%, C_{\mathrm{D}}=0.51$

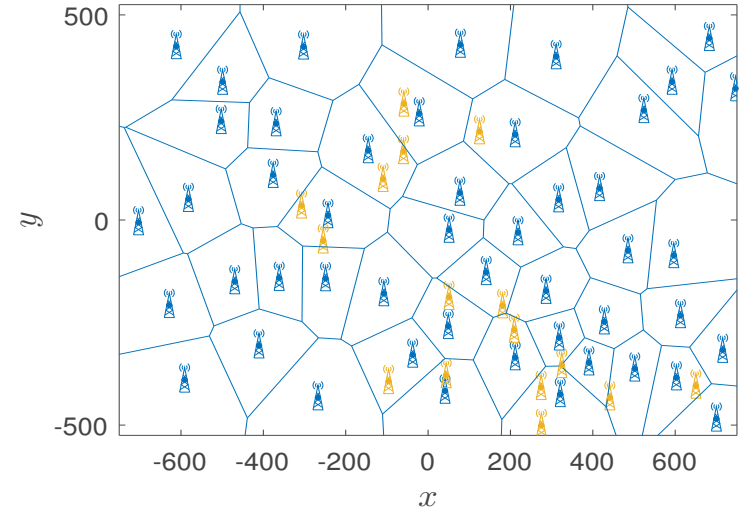

(b) $\rho=25 \%, C_{\mathrm{D}}=0.605$

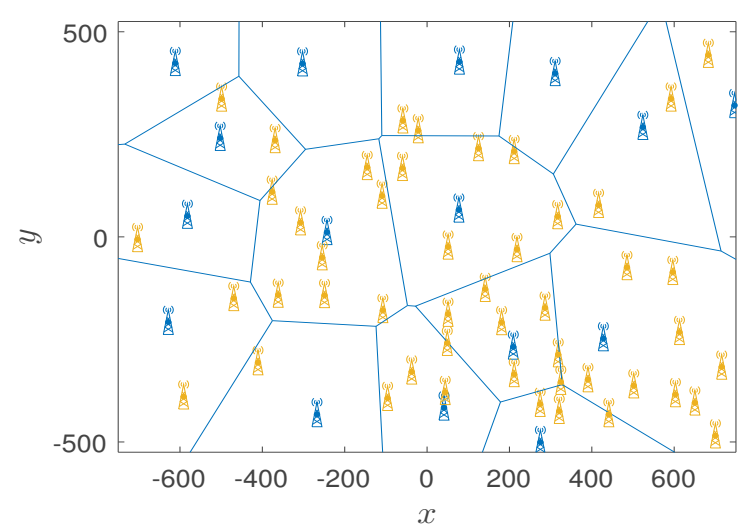

(d) $\rho=75 \%, C_{\mathrm{D}}=0.63$

Figure 6.6: Applying the semi-greedy deletion (SG) algorithm on real BS locations at different CSO percentages $\rho$. Blue and golden towers represent the active and inactive BSs, respectively. The BSs (GSM band $900 \mathrm{MHz}$ ) belong to the Vodafone operator. The region is $1500 \mathrm{~m} \times 1050 \mathrm{~m}$, in London, UK, centred at $\left(51.5136^{\circ} \mathrm{N}, 0.1342^{\circ} \mathrm{W}\right)$. This data is similar to that used in [13, Fig. 1]. 


\section{Chapter 7}

\section{Strategic Densification with UAV-BSs in Cellular Networks}

"Imagination is more important than

knowledge. Knowledge is limited. Imagination

encircles the world."

- Albert Einstein

\subsection{Introduction}

Beyond their traditional military use-cases, the unmanned aerial vehicles (UAVs), or drones, have recently found a wide variety of the civilian and commercial applications such as disaster response, photography, agriculture, retailing and e-commerce (package delivery), law enforcement, security, and surveillance. Further, employing the UAVs to act as flying BSs (UAV-BSs) in future cellular systems is a promising new evolution of wireless networks for the provision of on-demand coverage and capacity. Due to their mobility, the UAV-BSs attract the attention of the researchers to use them in the deployment of the futuristic flexible and agile mobile networks.

As mentioned before, cellular network densification (i.e., deploying more BSs) is an essential technique to accommodate the rapidly increasing demand for high system and per-user data rate [65-67]. Unconstrained location, easy relocation, and rapid deployment ability make the UAV-BSs more suitable than T-BSs (terrestrial BSs) 
for temporary densification to capture a sudden rise in traffic [68]. On the contrary, building permanent T-BSs is cost-ineffective: It requires obtaining a permit and high site rental costs [65]. Many of these BSs become underutilized during light traffic periods. Thus, instead of deploying excessive T-BSs to satisfy peak traffic demand, we advocate deploying on-demand UAV-BSs.

Most recent studies focused on finding an efficient placement of UAV-BSs where no T-BSs exist, for example, see [69-76]. With the existence of T-BSs, UAVs are employed in [77] as a static aerial relay and in [78] as a mobile aerial relay to assist the communication between a T-BS and a user terminal. In [79], a neural model is used to intelligently allocate multiple UAV-BSs to capacity-strained areas in a cellular network.

\subsection{Contribution}

Given a terrestrial network suffering from a temporary high demand for data rate, this chapter ${ }^{1}$ considers strategic placement of UAV-BSs to enhance both the capacity and the coverage probability. Inspired by improving the spatial regularity of the BS locations maximizes the coverage probability and the performance of the network [13, 23, 24, 59], we propose an aerial-terrestrial network planning approach that relies on the spatial structure of the network to strategically augment (find the best 3D locations) massive number of UAV-BSs to terrestrial network. We choose the horizontal location of the UAV-BSs such that they improve the spatial regularity of the network's BSs (the T-BSs and the projection of the UAV-BS locations on the ground). Figure 7.1 shows a graphical illustration of the strategic horizontal placement of the UAV-BSs. As for the altitude of the UAV-BSs, each one is adjusted

\footnotetext{
${ }^{1}$ This chapter has been published in the IEEE Wireless Communication Letters [80].
} 


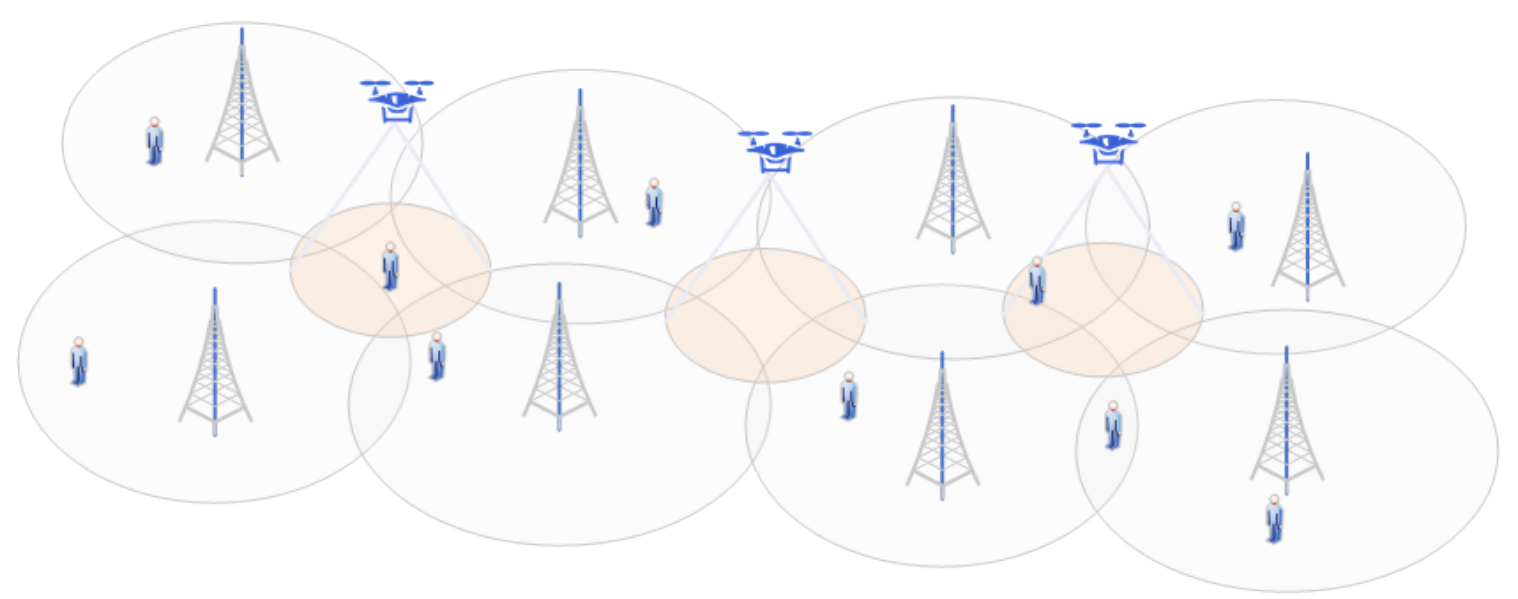

Figure 7.1: Graphical illustration of the strategic deployment of UAV-BSs in cellular networks.

based on the horizontal distance to its nearest BS for even better network throughput compared to random or fixed altitude cases. As such, we fill the coverage gaps and maximize the network's throughput.

Our main contribution is to propose the strategic placement of UAV-BSs over an already existing terrestrial network. Notably, although we put forward this study in the context of UAV-BS placement, it is a general large-scale network planning approach that could be used to design network densification regardless of the cell size or type. In this work, we do not aim to find the global optimal best-performance placement, but instead, aim to present a proof-of-concept and set in motion a novel research framework that will warrant further investigation. This work could be envisioned as the dual of the work presented in Chapter 6. They both are network planning problems that increase the spatial regularity of the network nodes to improve the performance. The problem in Chapter 6 is a cell switch-off (removal) problem, whereas the problem presented in this chapter is a cell deployment (switch-on) problem.

The organization of this chapter is as follows: We start by presenting the placement and the network performance metrics in Section 7.3. The system model is then 
considered in Section 7.4. The horizontal and the vertical strategic placement methods are presented in Section 7.5. Finally, the results and a discussion are presented in Section 7.6. The chapter is concluded in Section 7.7.

\subsection{Placement and Performance Metrics}

\subsubsection{Placement (Regularity) Metric}

To improve the spatial regularity, we need a metric to measure it. In this chapter, we use the edges of a Delaunay triangulation of the spatial positions of a set of BSs, which are random quantities, as a metric to characterize the spatial organization of that set of BSs $[23,41]$. Specifically, the coefficient of variation - the ratio of the standard deviation to the mean - of the edges of a Delaunay triangulation $\left(C_{\mathrm{D}}\right)$ is used to characterize the spatial regularity/irregularity of BS locations, which is explained with details in Chapter 2. We should remember that as $C_{\mathrm{D}}$ increases, the spatial irregularity increases (i.e., regularity decreases).

\subsubsection{Performance Metrics}

\section{Median SINR}

The signal-to-interference-plus-noise-ratio (SINR) value that $50 \%$ of the users in the system can achieve.

\section{Normalized Network Capacity}

The sum of rates of all users in the system in bits/Hz normalized by the sum-rate of a PPP-deployed network (deployed according to a PPP) before augmenting the UAV-BSs. 


\subsection{Downlink System Model}

We consider a network consisting of a set of T-BSs and a set of UAV-BSs. For both sets, unless otherwise stated, all parameters and specifications -including the terrestrial channel model - follow the urban macro (UMa) scenario [63]. For UAVBSs, we use the air-to-ground channel model in [70].

\section{System Setup}

We assume equal resource allocation scheme. Independent of network deployment, the users are spatially distributed according to a PPP with uniform data rate demand. Each user is connected to a BS (either aerial or terrestrial) that provides the strongest SINR. Neither fading nor shadowing is considered in this scenario. The UAV-BSs and the T-BSs are identical: They transmit at the same fixed power level, have the same carrier frequency, serve with a full buffer, are equipped with a single antenna, and so on. While each T-BS has an omni-directional antenna, a UAV-BS has a directional antenna (due to its high altitude and high line-of-sight opportunity) to reduce interfering with others.

\section{Air-to-Ground Channel Model}

We use a generic path loss model proposed in [70] for the air-to-ground channel. The model considers line-of-sight (LoS) and non-line-of-sight (NLoS) links as

$$
\begin{gathered}
\mathrm{PL}_{\mathrm{LoS}}=20 \log \left(\frac{4 \pi f_{c} d_{i j}}{c}\right)+\eta_{\mathrm{LoS}}+D\left(\phi_{i j}\right), \\
\mathrm{PL}_{\mathrm{NLoS}}=20 \log \left(\frac{4 \pi f_{c} d_{i j}}{c}\right)+\eta_{\mathrm{NLOS}}+D\left(\phi_{i j}\right) .
\end{gathered}
$$


The occurrence probability of the LoS link is given by

$$
\mathbb{P}_{\mathrm{LoS}}\left(\theta_{i j}\right)=\left(1+\alpha \cdot \exp \left(-\beta\left[\left(\theta_{i j}-\alpha\right]\right)\right)^{-1}\right.
$$

and the NLoS link by $\mathbb{P}_{\mathrm{NLoS}}\left(\theta_{i j}\right)=1-\mathbb{P}_{\mathrm{LoS}}\left(\theta_{i j}\right)$, where $\theta_{i j}=\sin ^{-1}\left(h_{j} / d_{i j}\right)$ is the elevation angle (degrees) between a user $i$ and UAV-BS $j$. Note that $h_{j}$ is the altitude of the UAV-BS, $d_{i j}=\left\|u_{i}-l_{j}\right\|$ is the Euclidean distance between a user located at $u_{i} \in \mathbb{R}^{3}$ and a UAV-BS located at $l_{j} \in \mathbb{R}^{3}$, and $f_{c}$ is the carrier frequency, where $c$ is the speed of light. Also, $\eta_{\mathrm{LoS}}$ and $\eta_{\mathrm{NLoS}}(\mathrm{dB})$ are environment-dependent losses corresponding to the LoS and NLoS links, respectively. $\alpha$ and $\beta$ are other environment-dependent parameters. The antenna of the UAV-BSs is perpendicular to the ground, and its directivity gain $D\left(\phi_{i j}\right)$ is specified [63] as

$$
D\left(\phi_{i j}\right)=\min \left[12\left(\frac{\phi_{i j}}{15^{\circ}}\right)^{2}, 20 \mathrm{~dB}\right] \text {, }
$$

where $\phi_{i j}=90-\theta_{i j}$.

The probabilistic path loss can be written as

$$
\mathrm{PL}=\mathrm{PL}_{\mathrm{LoS}} \times \mathbb{P}_{\mathrm{LoS}}\left(\theta_{i j}\right)+\mathrm{PL}_{\mathrm{NLoS}} \times \mathbb{P}_{\mathrm{NLoS}}\left(\theta_{i j}\right)
$$

\section{Terrestrial-BS Deployment}

The deployment of the T-BSs is modelled using a PTL: a repulsive point process that provides variable spatial regularity. As provided in Chapter 4, the uniform PTL is a result of the independent uniform random displacement on a disk for each point (BS location) of a triangular lattice from its original location [41]. 


\subsection{Strategic Placement Method}

The best horizontal placement of the UAV-BSs must decrease the spatial irregularity of the network, which can be achieved by spreading out (to the greatest possible extent) the horizontal location of the new UAV-BSs from each other and from the existing BSs. One approach to achieving the highest spatial regularity, i.e., the farthest distribution of the points, is to maximize the sum of the distance between the nodes [81] as we formulate in (7.6). As for the altitude, it must be chosen such that it provides the highest signal strength inside the targeted area without interfering with the surrounding BSs.

\section{Horizontal Placement}

Given a set $\mathcal{A}=\left\{a_{1}, a_{2}, \ldots, a_{\mathrm{N}_{\mathrm{t}}}\right\} \subset \mathbb{R}^{2}$ of the locations of $\mathrm{N}_{\mathrm{t}}$ terrestrial BSs and a set $C=\left\{c_{1}, c_{2}, \ldots, c_{N_{d}}\right\} \subset \mathbb{R}^{2}$ representing $\mathrm{N}_{\mathrm{d}}$ predefined potential horizontal locations of UAV-BSs, we aim to select $M$ locations from the set $C$ for UAV-BSs to hover over such that the spatial regularity of all the nodes is maximized. The percentage of the augmented UAV-BSs to the T-BSs is

$$
\zeta=\left(\frac{M}{\mathrm{~N}_{\mathrm{t}}}\right) \times 100 \% .
$$

Let the set $\mathcal{N}$ be the concatenation of the sets $\mathcal{A}$ and $\mathcal{C}$. Formally, $\mathcal{N}=\mathcal{A} \| C=$ $\left\{a_{1}, a_{2}, \ldots, a_{N_{\mathrm{t}}}, c_{1}, c_{2}, \ldots, c_{\mathrm{N}_{\mathrm{d}}}\right\} \subset \mathbb{R}^{2}$. Members of $\mathcal{N}$ are indexed by $j$, and for simplicity we refer to them only by their indexes. The members of $\mathcal{N}$ with index $j \leq N_{t}$ belong to $\mathcal{A}$ and those with index $j>\mathrm{N}_{\mathrm{t}}$ belong to $\mathcal{C}$.

A binary quadratic formulation for maximizing the sum of the distance between 
the nodes is as follows:

$$
\begin{array}{ll}
\underset{z_{j}}{\operatorname{maximize}} & \sum_{i \in \mathcal{N}} \sum_{j \in \mathcal{N}} w_{i j} z_{i} z_{j} \\
\text { subject to } & \sum_{j \in \mathcal{N}} z_{j}=p \\
& z_{j} \in\{0,1\}, \quad j \in \mathcal{N} \\
& z_{j}=1, \quad \forall j \leq \mathrm{N}_{\mathrm{t}}, \quad j \in \mathcal{N} .
\end{array}
$$

The Boolean variable $z_{j}$ indicates whether a node location is selected. The distance between the node $i$ and $j$ is defined by $w_{i j}$, and of course $w_{i i}=0$. The total number of the selected nodes $p=\mathrm{N}_{\mathrm{t}}+M$. The constraint (7.6d) forces all terrestrial BSs to be selected.

The problem is difficult to solve optimally on a large scale. (It is an NP-hard as similar problems are known to be NP-hard, e.g., see [81].) Therefore, we propose a greedy-type heuristic algorithm to deal with it. The proposed algorithm consists of two main sets: the candidates set $\mathcal{C}$ and the solution set $\mathcal{B}$. The candidates set represents the possible horizontal locations of UAV-BSs, while the solution set contains the T-BS locations as well as the already chosen UAV-BS horizontal location. The solution set of this algorithm is initialized by the locations of the TBSs $\mathcal{A}$. Then, at each iteration, a new UAV-BS horizontal location that is the furthest apart from all points in this solution set is added until the required number of the UAV-BSs $M$ is achieved. Algorithm 3 illustrates the pseudo-code of the UAV-BSs' horizontal placement algorithm. Its complexity at most is $O\left(N^{3}\right)$, where $N=|\mathcal{N}|=\mathrm{N}_{\mathrm{t}}+\mathrm{N}_{\mathrm{d}}$. A notation $\operatorname{dist}\left(a_{i}, a_{j}\right)=\left\|a_{i}-a_{j}\right\|$ denotes the Euclidean distance between the points $a_{i}$ and $a_{j}$, and the notation $\operatorname{dist}\left(a_{i}, \mathcal{B}\right)$ denotes the distance between the point $a_{i}$ and its nearest neighbour point in the set $\mathcal{B}$. Namely, 
$\operatorname{dist}\left(a_{i}, \mathcal{B}\right)=\min \left\{\operatorname{dist}\left(a_{i}, a_{j}\right): a_{j} \in \mathcal{B}, a_{j} \neq a_{i}, \forall j\right\}$. Also, $|\mathcal{B}|$ indicates the number of points in the set $\mathcal{B}$. Figure 7.2 depicts the horizontal placement using Algorithm 3. In practical implementations, a cloud-based entity [68] could be used to implement as well as to collect, store, and manage the information required to execute this Algorithm.

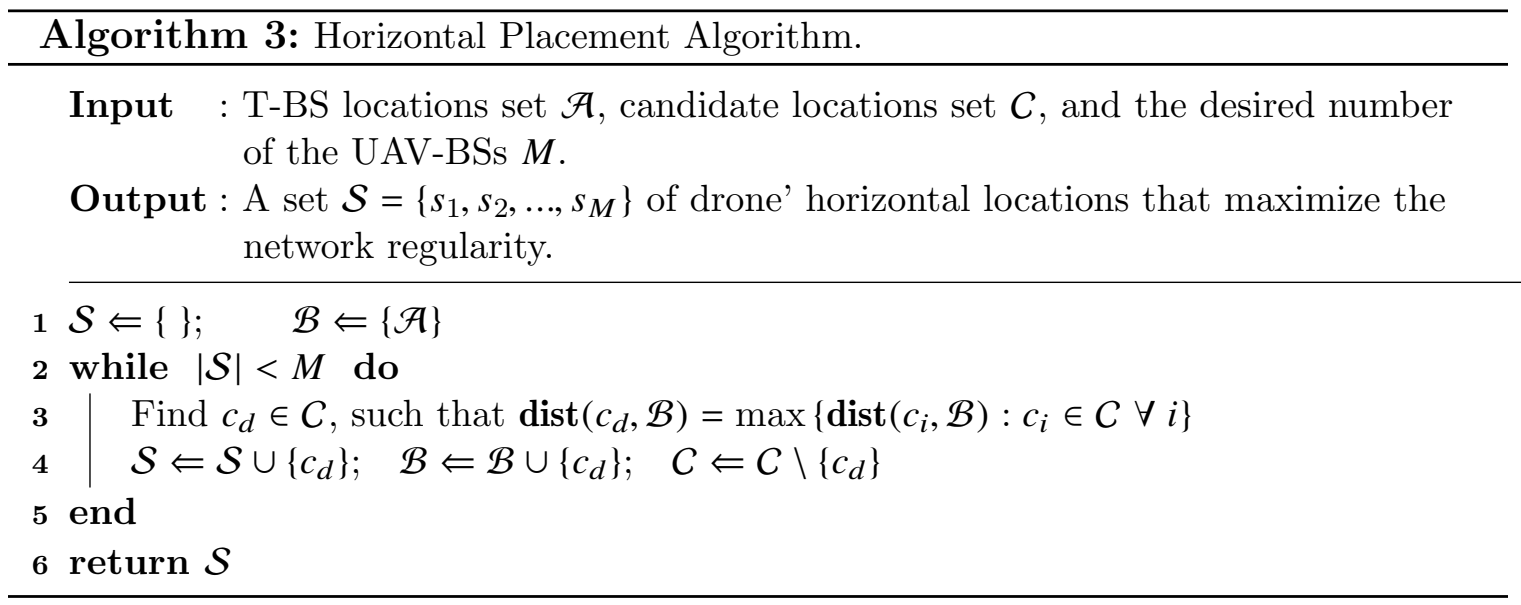

\section{Vertical Placement}

We adjust the altitude of the UAV-BSs $\left(\mathcal{H}=\left\{h_{1}, h_{2}, \ldots, h_{M}\right\}\right)$ to provide maximum coverage. We approximate the coverage region for each UAV-BS to be a disk. The radius of the coverage disk $R_{j}$ is half the horizontal distance to the nearest neighbour BS, either another UAV-BS or a T-BS. From [71], we observe that the optimal altitude $h_{j}$ depends only on the radius of the coverage region $R_{j}$ and the optimal elevation angle $\theta_{\mathrm{opt}}$. It can be found using [71]:

$$
h_{j}=k \times R_{j} \tan \left(\theta_{\mathrm{opt}}\right) \text {, }
$$




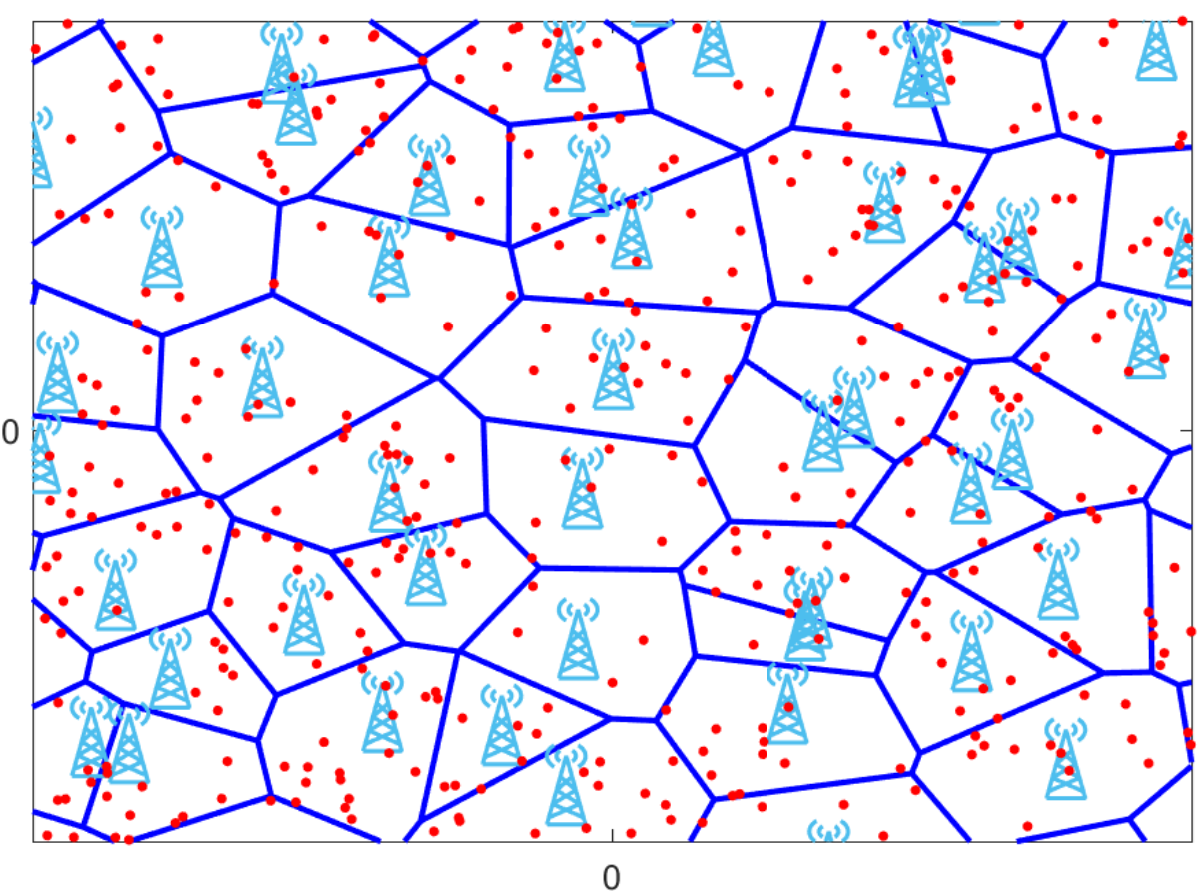

(a)

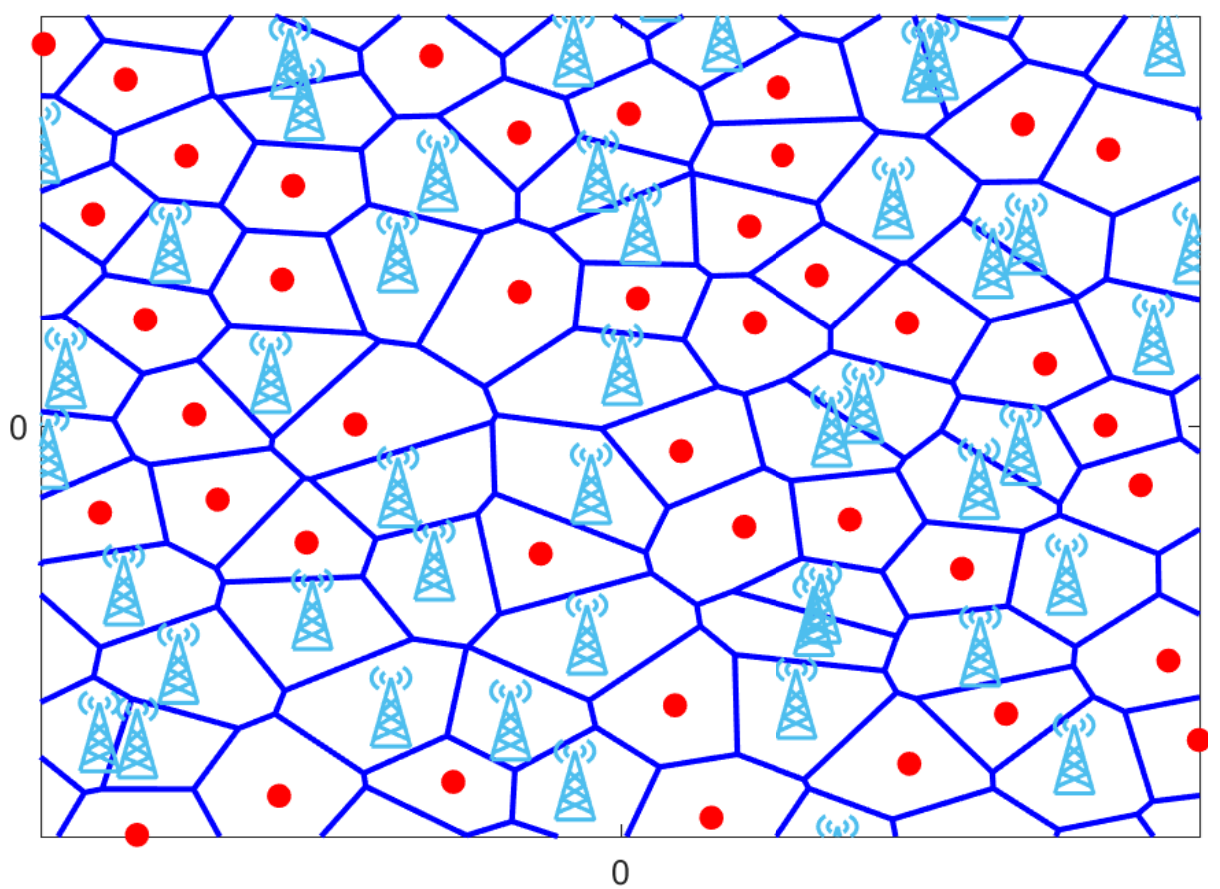

(b)

Figure 7.2: (a) Arbitrary T-BSs deployment (blue towers) with random candidate location (red dots). (b) T-BSs with the projection of the UAV-BSs on the ground (red disks) as selected from the random candidates using Algorithm 1. 
where $0<k \leq 1$ is a scaling factor used to reduce the altitude of the UAV-BS so as to back off the interference. In this chapter, using a line search method, we select $k=0.6$ for all UAV-BSs.

We refer to the strategic placement above as Strategy I; it is a placement based merely on the long-time average of the user locations. To enrich the discussion, we also introduce three slightly different placement strategies.

1) Strategy II: random horizontal placement with fixed altitude.

2) Strategy III: strategic horizontal placement, as in Algorithm 3, with random altitude.

3) Strategy IV: strategic horizontal placement, as in Algorithm 3, but with further readjustment flexibility that allows the horizontal location of the UAV-BS to be located exactly in the centre of the user locations within its coverage region, defined by its Voronoi cell. The altitude is then found according to (7.7).

\subsection{Results}

We carry out the simulation over 1000 Monte Carlo runs. We execute the experiment for T-BSs deployed with four different spatial regularity values — similar to Figure 2.3 ( [59, Figure 1]) — as generated using the PTL model. We consider a network of around 100 T-BSs deployed with an average inter-site distance of $500 \mathrm{~m}$ [63], which is established on an approximately $4.6 \mathrm{~km} \times 4.6 \mathrm{~km}$ geographical region. An average of 2000 random users are uniformly distributed over the whole region, but only users within the central area of $2.8 \mathrm{~km} \times 2.8 \mathrm{~km}$ are considered in the simulation to eliminate the boundary effects. For the same reason, the set of the candidate horizontal locations of the UAV-BSs - a large set of discrete random locations - is generated inside the convex haul of T-BS locations. Network parameters are set according to the 
UMa scenario [63]. Since we consider an urban environment, the parameters of the air-to-ground channel model $\left(\alpha, \beta, \eta_{\mathrm{LoS}}, \eta_{\mathrm{NLOS}}\right)$ are given by $(9.6117,0.1581,1 \mathrm{~dB}$, $20 \mathrm{~dB}$ ) [70,71], respectively. Hence, the optimal elevation angle $\theta_{\mathrm{opt}}=42.44^{\circ}[73]$ for this environment, as it is found by numerically solving [71, Eq. (13)].

In Figures 7.3-7.5, each sub-figure represents a specific spatial deployment of TBSs with certain regularity values $\left(\boldsymbol{C}_{\mathrm{D}}(\right.$ in $\left.)\right)$ - similar to Figure $2.3([59$, Figure 1$])$ and depicts four placement strategies. The figures show that the performance of the network depends on its spatial structure. In Figure 7.3, we see that the strategic horizontal placement of the UAV-BSs improves the spatial regularity of the network $\left(C_{\mathrm{D}}\right.$ (out)). As a particular case, adding more points to an entirely regular (i.e., hexagonal layout) or highly regular spatial pattern has to result in a deterioration of the overall regularity, which is the case in Figures 7.3(a) and 7.3(b). However, this may be the best achievable regularity when more points are added to a very regular spatial pattern. (Indeed, the best placement algorithm either much improves the regularity when the BS deployment is highly irregular or provides the least degradation in regularity when the BS deployment is very regular.) The spatial regularity of the network is similar for both Strategy I and Strategy IV, i.e., the average horizontal location of the UAV-BSs over all realizations is the same for both strategies.

Figures 7.4 and 7.5 show the median SINR and the normalized sum-rate as a function of the UAV-BSs augmentation ratio ${ }^{2}$. They indicate that Strategy IV provides best median SINR and normalized sum-rate followed by Strategy I. For example, for a PPP-deployed terrestrial network (Figures 7.4(d) and 7.5(d)), the median SINR and the normalized sum-rate for Strategy IV and Strategy I are (10.63 dB, 2.99) and (9.05 dB, 2.48), respectively. Remarkably, they both are much better than Strategy II (4.8

\footnotetext{
${ }^{2} \mathrm{UAV}-\mathrm{BSs}$ augmentation ratio could be greater than $1(\zeta>1)$, i.e., more UAV-BSs than T-BSs. Hence, we expect the performance continues to improve until it reaches either a peak - and then declines - or a plateau; the exact behaviour critically depends on the terrestrial and aerial channel models.
} 
$\mathrm{dB}, 1.85)$. In fact, Strategy IV simply provides further tuning to the statistical approach of Strategy I. The disadvantage of Strategy IV is that it requires instantaneous UAV-BS movement to track the mobile users. The frequent repositioning of the UAVs may not be energy efficient and would be computationally more expensive. (In this strategy, the additional complexity includes the complexity of Voronoi tessellation $O(p \log p)$, where $p$ is the number of the BSs and the complexity of point-in-polygon algorithm $O(n \log n)$, where $n$ is the number of users.)

For a terrestrial network deployed according to a hexagonal layout, we observe that adding more UAV-BSs results in a better sum-rate even for Strategy II, although the median SINR deteriorates - look at Figure 7.4(a) and Figure 7.5(a) together. Noteworthy, adding more nodes to an entirely regular deployment deteriorates the regularity and the SINR but provides more capacity.

Given the required increase in the capacity, we can estimate how many UAVBSs are needed using Figure 7.5. For example, the sum-rate of a PPP-deployed network (Figure $7.5(\mathrm{~d})$ ) can be doubled by adding UAV-BSs at the augmentation ratio $\zeta=0.48$

\subsection{Concluding Remarks}

This chapter has advanced the previous knowledge gained on the 3D placement of the UAV-BSs. It provided a framework for improving network capacity by strategically placing UAV-BSs to assist a cellular network. Based on the maximization of the spatial regularity of the network's structure, this chapter proposed a novel large-scale network planning approach that helps to decide where UAV-BSs hover to maintain connectivity, fill the coverage holes and to boost network capacity. We showed that 


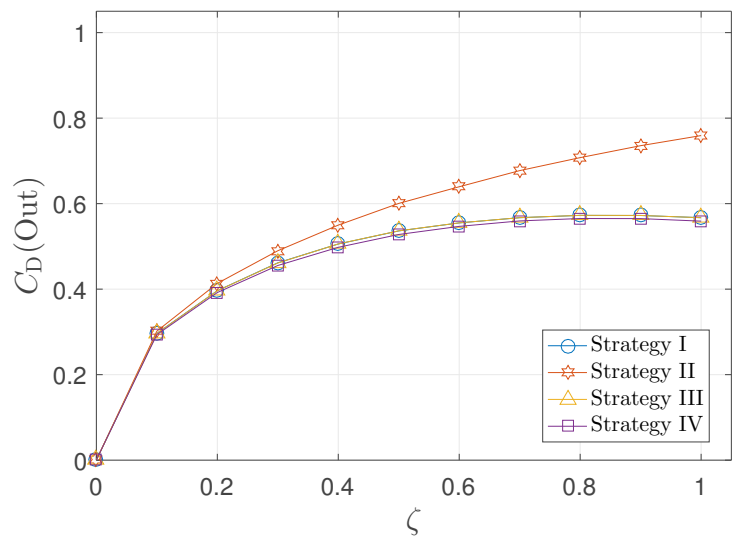

(a) $C_{\mathrm{D}}$ (in) $=0$

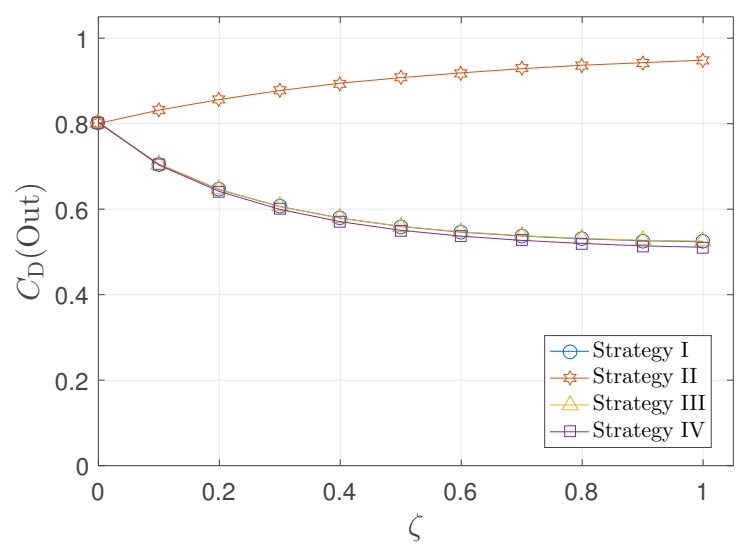

(c) $C_{\mathrm{D}}($ in $)=0.8$

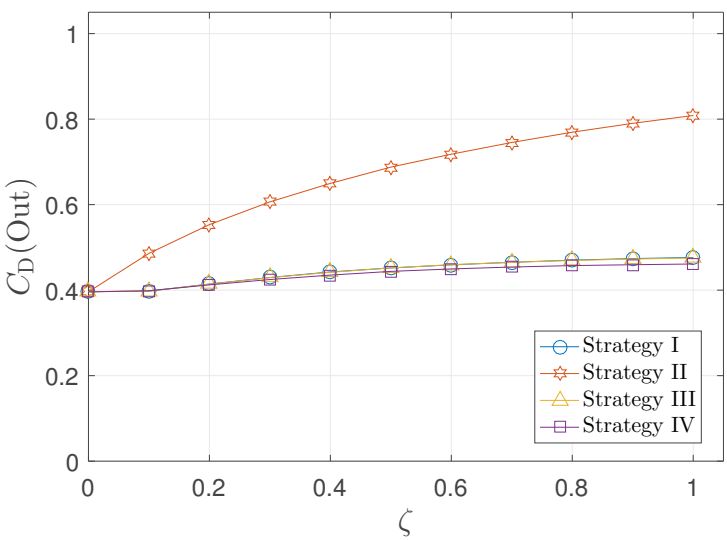

(b) $C_{\mathrm{D}}($ in $)=0.4$

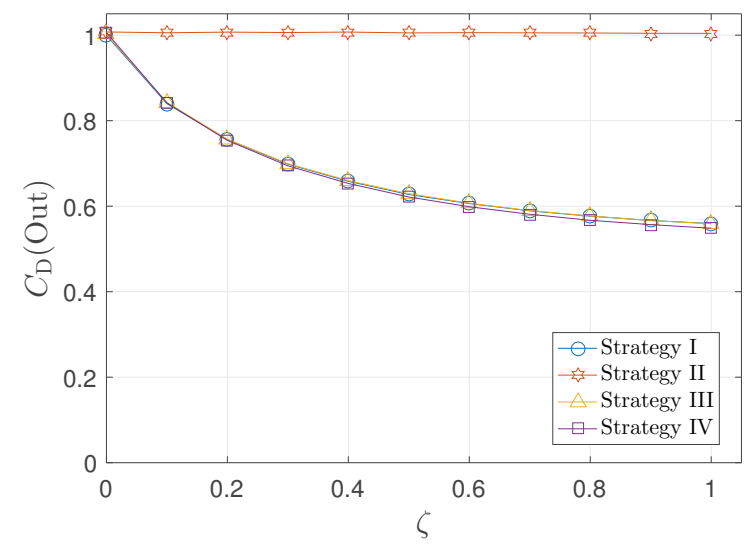

(d) $C_{\mathrm{D}}($ in $)=1$

Figure 7.3: The $C_{\mathrm{D}}$ (out) of the network as a function of the augmentation percentage $\zeta$. Each sub-figure represents a specific regularity value $C_{\mathrm{D}}$ (in) of the original network deployment (similar to [59, Fig. 1]). 


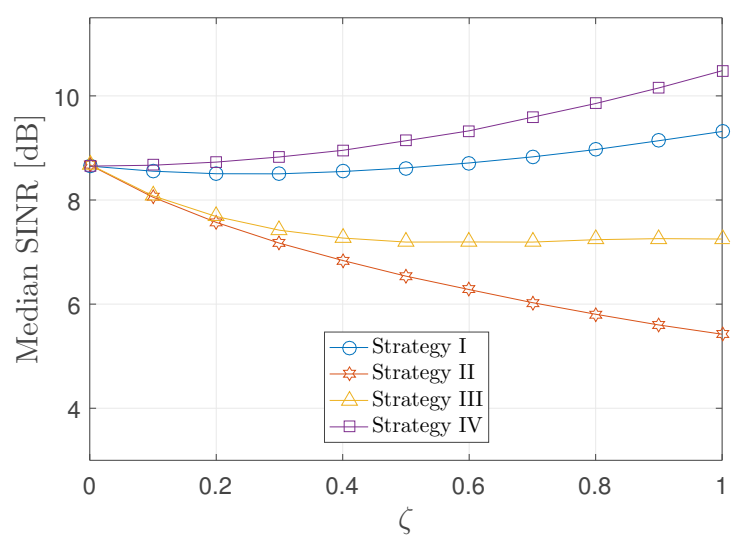

(a) $C_{\mathrm{D}}($ in $)=0$

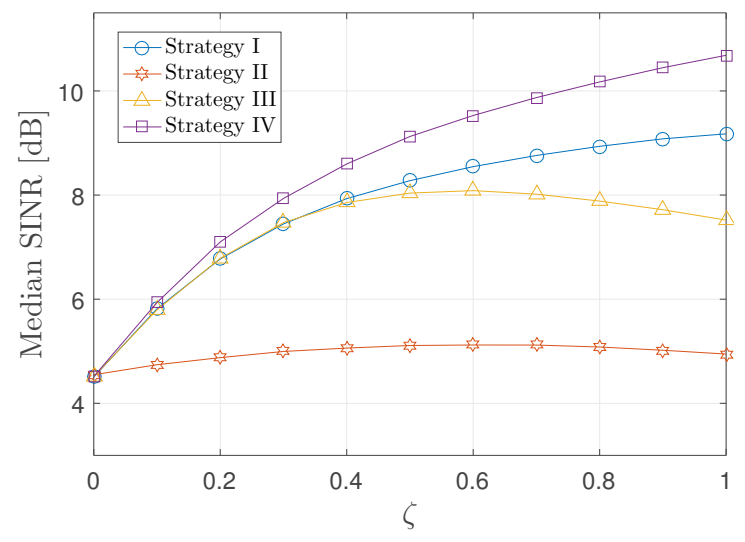

(c) $C_{\mathrm{D}}($ in $)=0.8$

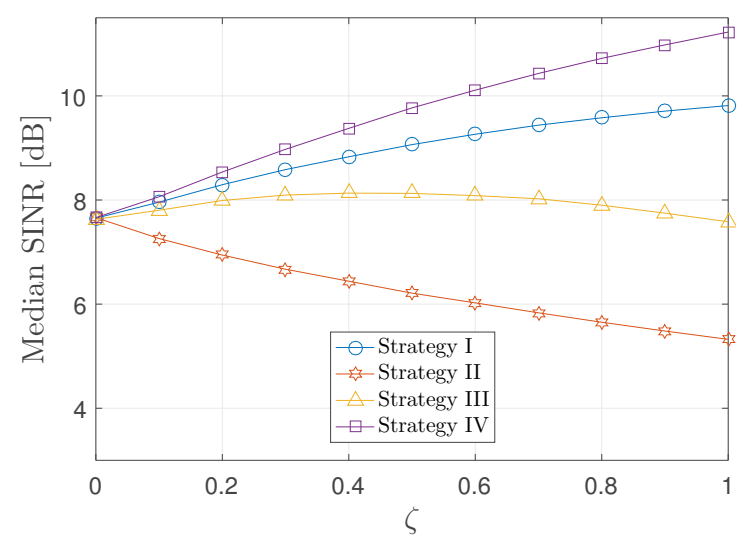

(b) $C_{\mathrm{D}}(\mathrm{in})=0.4$

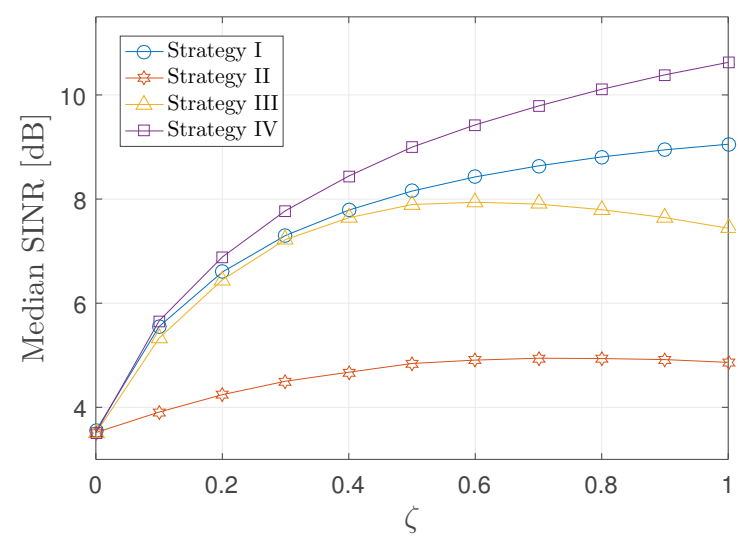

(d) $C_{\mathrm{D}}(\mathrm{in})=1(\mathrm{PPP})$

Figure 7.4: The median SINR as a function of the augmentation percentage $\zeta$ for different network deployment regularity $C_{\mathrm{D}}(\mathrm{in})$. 


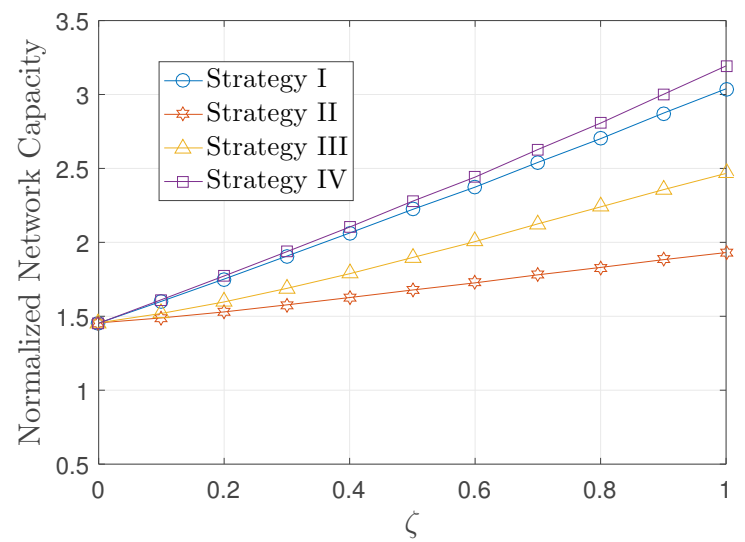

(a) $C_{\mathrm{D}}$ (in) $=0$

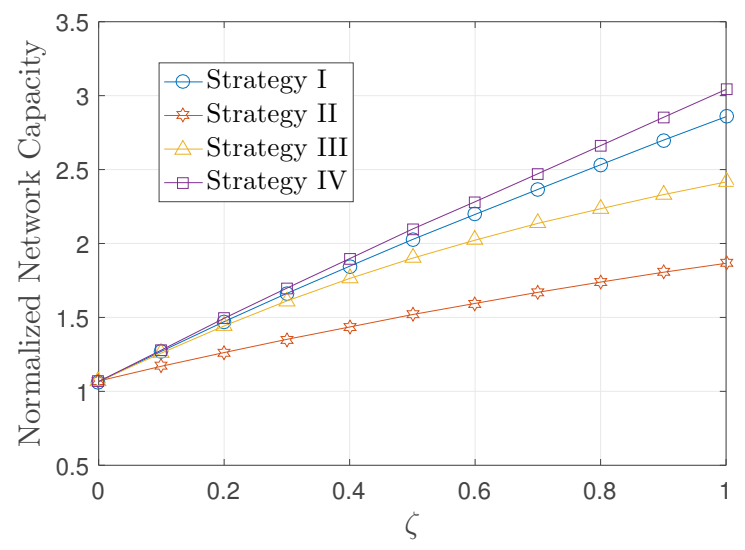

(c) $C_{\mathrm{D}}($ in $)=0.8$

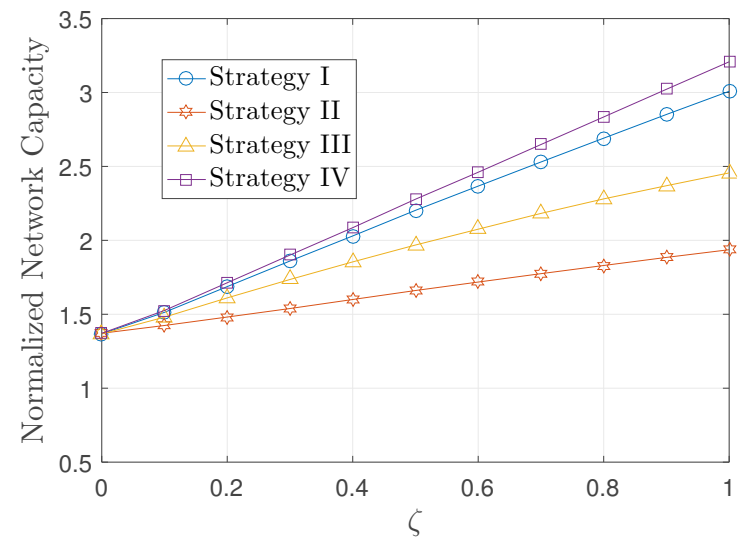

(b) $C_{\mathrm{D}}$ (in) $=0.4$

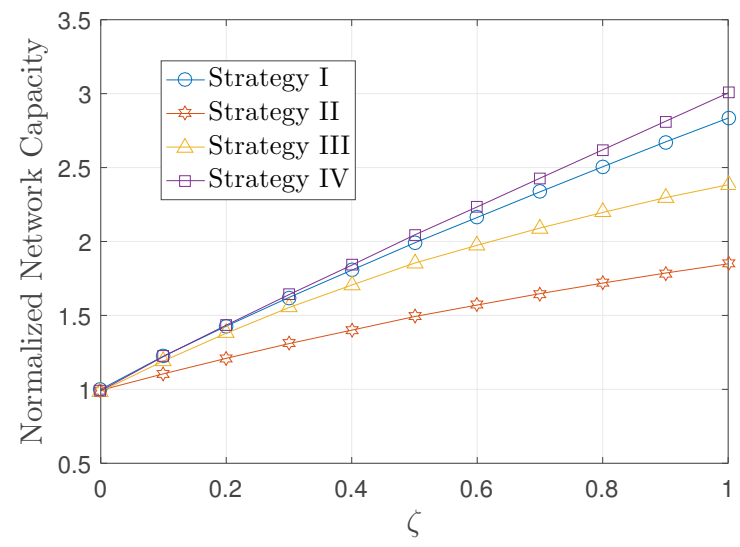

(d) $C_{\mathrm{D}}($ in $)=1(\mathrm{PPP})$

Figure 7.5: Normalized network capacity as a function of the UAV-BS augmentation percentages $\zeta$ for different network deployment regularity $C_{\mathrm{D}}$ (in). 
the best place for UAV-BSs is where their projection on the ground improves the spatial regularity of the network. We observed that even the random augmentation of the UAV-BSs enhances network throughput (yet it could be associated with deterioration of the spatial regularity and the SINR); the strategic augmentation maximizes this throughput. Allowing additional horizontal location adjustment based on the exact location of the users provides further gain. However, in practise, we need to trade off the energy consumption and computation time with the throughput reward. Future works could extend this framework to the non-uniform distribution of the users. 


\section{Conclusion and Future Work}




\section{Chapter 8}

\section{Conclusion and Future Work}

"Our imagination is the only limit to what we can hope to have in the future."

- Charles F. Kettering

\subsection{Summary and Conclusion}

A key enabler for the next generation cellular networks to support the explosive growth of traffic demand is the intensive deployment of the BSs. Indeed, the efficient utilization of this additional infrastructure requires realistic spatial modelling for the existing BS locations, a keen understanding of their spatial relationship, and strategic network planning. The first part of this thesis proposed several mathematical tools to measure the spatial relationship among the BSs. The best one among these tools is used to estimate the network performance, to fit point processes to real deployment of BSs, and to identify the realistic model for the BS deployment. Then, in the second part, the insights from the first part are used to propose a novel cell switch-off and a strategic UAV-BS placement approaches. We now summarize each chapter of this thesis and its key contributions.

In Chapter 1, we identified the two most prevalent models to simulate the spatial geometry of the BSs in the cellular systems, which are the TL and PPP. However, 
real deployments of the BSs are neither perfectly regular nor completely irregular (random). More specifically, the deployment of the cellular networks is not entirely regular, on the one hand, because the layout of the natural and artificial physical features (buildings, landscape, rivers, population densities, and traffic demands) of the served geographical region prevents the operators from placing the BSs on a triangular lattice. On the other hand, network operators tend to put the BSs as far away as possible from each other to reduce the interference and extend the coverage. The location of each BS is influenced by the location of the others. The BSs are not arbitrarily deployed to follow a PPP, but they repel each other. In reality, therefore, the spatial structure of the BSs takes place somewhere in between these two opposite ends of the scale. To account for the repulsion of the spatial structure of the cellular systems, it is more accurate to use repulsive point processes to model the locations of the BSs. Chapter 1 explained thoroughly why the location of the BSs in wireless networks should be modelled as repulsive random point processes with an amount of regularity that is tunable between that of a TL and its counterpart, PPP. Actually, we found out how much is difficult difficult to quantify the regularity or compare different repulsive point processes. Chapter 1 highlighted the research directions, scope, objectives, and contributions of this thesis.

In Chapter 2, we proposed three metrics based on the CoV of some geometric properties of the spatial distribution of the BSs to measure their regularity. We compared these metrics in the first part of this thesis, and the best performing metric is used is used in the following chapters for measuring the regularity, mapping point processes to each other, approximating the network performance, and fitting BS deployments to models.

Chapter 3 examined the geometry-based metrics and showed that these metrics could be used as yardsticks to measure the spatial regularity of the BSs and as network 
performance indicators. Three hard-core point processes, which were used in this test, were compared regarding their spatial regularity range and the density of the generated points. The results show that the SSI is the best among these hard-core point processes for modelling the BS locations.

In Chapter 4, the CoV-based metrics are used to measure the regularity of two common types of PTL: uniform on disc and Gaussian. From these measurements, it was possible to derive a simple formula that allows matching and interchanging the two PTL models, within about $0.1 \mathrm{~dB}$ SIR error. It is a novel approach for mapping between different point processes using CoV-based metrics as an intermediate step. This chapter paved the way for the researchers to map the RPPs to each other. Chapter 4 gave qualitative and quantitative arguments supporting the use of PTLs for modelling BS deployments with any amount of spatial regularity, which is useful notably in modelling different tiers of HetNets.

Chapter 5 concentrated on finding the best regularity metric to quantify the spatial relationship among the BSs in wireless cellular networks and its possible applications. The results in this chapter indicated that the $\mathrm{CoV}$ of the length of the edges of Delaunay triangulation of the BSs locations is the best to quantify the spatial regularity among these BSs. The results showed that this geometry-based scalar metric is a robust indicator for the network performance. This chapter exploited the similarity between the coverage curves of the different BSs topologies and use the best metric to approximate the performance of one network deployment based on a known performance reference network. Using the best metric, this chapter described a novel practical technique for fitting spatial point processes models to spatial point pattern representing actual or simulated BSs locations. This chapter supports the results of Chapter 4 of advocating the use of the PTLs for modelling the spatial deployment of BSs. 
Chapter 6 considered the CSO, a promising approach to reduce the energy consumption in cellular networks. This chapter set a new CSO research direction that focused on saving energy and jointly increasing the performance of a network by switching off some cells to maximize the spatial regularity of the remaining active cells. The BSs were deployed with variable amounts of spatial regularity. That is to say; this work expanded the CSO literature by modelling BSs using RPPs. Three greedy algorithms were proposed for tackling this new problem. The results revealed that improving the spatial regularity using a greedy algorithm leads to either (i) much extra energy saving while maintaining network performance or (ii) saving the same amount of energy as the random CSO with better network performance.

Chapter 7 investigated the use of UAV-BSs for the provision of on-demand high data rates in cellular networks. While many studies have explored deploying UAVBSs in a green field - no existence of T-BSs, this work investigated the deployment of UAV-BSs in the presence of a terrestrial network. The purpose of this chapter is

twofold: 1) to provide supply-side estimation for how many UAV-BSs are needed to support a terrestrial network to achieve a particular quality of service and 2) to investigate where these UAV-BSs should hover. This chapter proposed a novel stochastic geometry-based network planning approach that focuses on the structure of the network to find strategic placement of multiple UAV-BSs in a large-scale network.

\subsection{Potential Future Works}

This work could be extended in many directions. Here we propose two important extensions. First is to use the hard-core point processes as CSO algorithms and to use the greedy algorithms as hard-core models for the BSs locations. Second is to extend the UAV-BSs strategic placement framework to the non-uniform demand scenario. 


\subsubsection{New Hard-Core Models and CSO Algorithms}

An important observation is that there is a strong similarity between the hardcore point processes presented in Chapter 3 for modelling the BSs locations and the greedy algorithms presented in Chapter 6 as CSO algorithms. Indeed, we can employ greedy algorithms (greedy construction (GC), greedy deletion (GD), and semi-greedy deletion $(\mathrm{SG})$ ) to generate new hard-core point processes. On the other hand, we can use the generating algorithms of well-known hard-core point processes (MHC-I, MHC-II, SSI) to work as CSO algorithms.

While the hard-core distance controls the number of the removed points (density) and the degree of repulsion in the hard-core point processes, the number of the points that are recommended to be removed controls the density and the degree of repulsion of the remaining points in the greedy algorithms.

Greedy algorithms would generate spatial patterns with higher regularity than the hard-core point processes MHC-I, MHC-II, and SSI. It would be interesting to investigate using greedy algorithms as models for the BSs deployments and to find analytical expressions for the SINR and the coverage probability. Such mathematical expressions should be functions of the number of the removed points (i.e., switched off cells) from the original PPP model. Before that, we need to determine the probability distribution function of the distance between any typical user and the BSs as a function of the number of the removed points. Another interesting research problem is to examine the suitability of the hard-core point processes as CSO algorithms. 


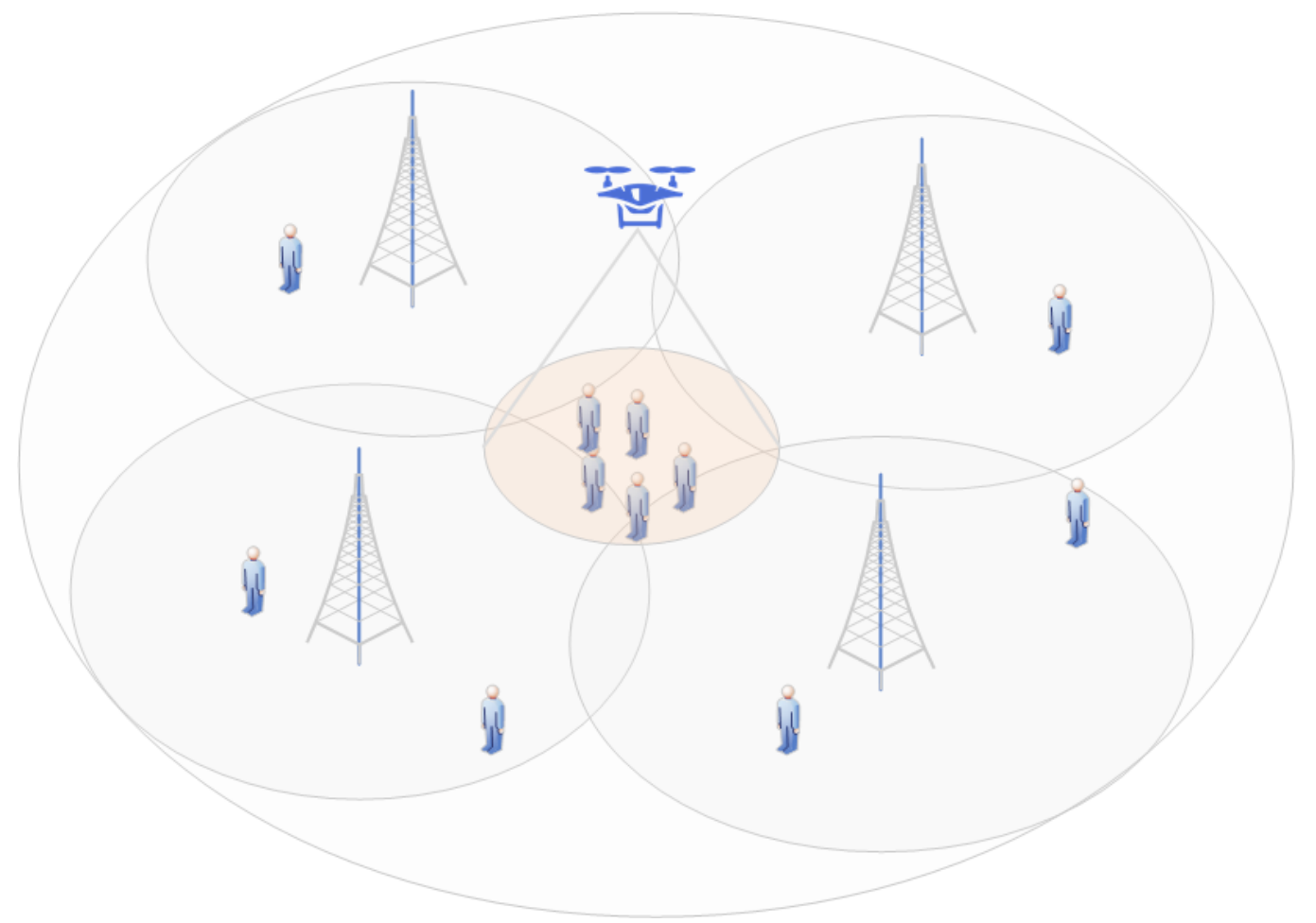

Figure 8.1: Graphical illustration of UAV-BS deployment in a wireless cellular networks with spatially heterogeneous user distribution.

\subsubsection{Large-Scale UAV-BS Deployment in Wireless Cellu- lar Networks with Spatially Heterogeneous Traffic De- mand}

In Chapter 7, we proposed a large-scale network planning framework that depends on the network structure - which is equivalent to placement based on the long-term averaging of the user locations - to strategically augment massive number of UAVBSs into a terrestrial network. Our work in Chapter 7 is confined to the uniform data demand over a whole geographical region. However, it is important to study the non-uniform traffic demand.

Many ways are possible to handle the non-uniformity of the traffic demand. One method could be by assigning weights to the users based on their data rate demand. 
Another method is modelling the non-uniform demand as clusters of users. In the latter case, we can employ machine learning-based tools to place the UAV-BSs at the cluster head. (Indeed, the operator would reap more benefits in the non-uniform (e.g., cluster user distribution) scenario since UAV-BSs could locate directly above the cluster head. This results in better network performance and fewer number of UAV-BSs would be used to serve the same number of users.) Adjusting the altitude of the UAV-BS should be based on the size of the cluster, which can reduce interference and fine-tune the links between users and the UAV-BS assigned to their cluster. Therefore, we may also need a sophisticated heuristic algorithm to adjust the altitude in the non-uniform scenario. The extension of our previous work to non-uniform user distribution would further advance the 3D placement of the UAV-BSs. 


\section{List of References}

[1] Ericsson, "Ericsson mobility report," tech. rep., June 2017.

[2] Cisco, "Cisco Visual Networking Index: Forecast and Methodology, 2016-2021," June 2017.

[3] QUALCOMM, "Rising to Meet the 1000x Mobile Data Challenge." 2012.

[4] J. G. Andrews, R. K. Ganti, M. Haenggi, N. Jindal, and S. Weber, "A primer on spatial modeling and analysis in wireless networks," IEEE Commun. Mag., vol. 48, pp. 156-163, Nov. 2010.

[5] M. Haenggi, Stochastic Geometry for Wireless Networks. Cambridge University Press, 2012.

[6] J. Møller and D. Stoyan, "Stochastic geometry and random tessellations," tech. rep., Dept. of Math. Sci., Aalborg University, 2007.

[7] Z. Zhao, S. S. Szyszkowicz, T. Beitelmal, and H. Yanikomeroglu, "Spatial clustering in slotted ALOHA two-hop random access for machine type communication," in IEEE Global Commun. Conf. (GLOBECOM), Dec. 2016.

[8] A. Guo and M. Haenggi, "Asymptotic deployment gain: A simple approach to characterize the SINR distribution in general cellular networks," IEEE Trans. Commun., vol. 63, pp. 962-976, Mar. 2015.

[9] F. Baccelli, M. Klein, M. Lebourges, and S. Zuyev, "Stochastic geometry and architecture of communication networks," Telecommunication Systems, vol. 7, pp. 209-227, June 1997.

[10] T. X. Brown, "Cellular performance bounds via shotgun cellular systems," IEEE J. Select. Areas Commun., vol. 18, pp. 2443-2455, Nov. 2000.

[11] H. S. Dhillon, R. K. Ganti, F. Baccelli, and J. G. Andrews, "Modeling and analysis of K-tier downlink heterogeneous cellular networks," IEEE J. Sel. Areas Commun., vol. 30, pp. 550-560, Apr. 2012.

[12] J. Andrews, F. Baccelli, and R. Ganti, "A tractable approach to coverage and rate in cellular networks," IEEE Trans. Commun., vol. 59, pp. 3122-3134, Nov. 2011. 
[13] A. Guo and M. Haenggi, "Spatial stochastic models and metrics for the structure of base stations in cellular networks," IEEE Trans. Wireless Commun., vol. 12, pp. 5800-5812, Nov. 2013.

[14] J. Andrews, "Seven ways that HetNets are a cellular paradigm shift," IEEE Commun. Mag., vol. 51, pp. 136-144, Mar. 2013.

[15] D. Taylor, H. Dhillon, T. Novlan, and J. Andrews, "Pairwise interaction processes for modeling cellular network topology," in IEEE Global Commun. Conf. (GLOBECOM), pp. 4524-4529, Dec. 2012.

[16] Y. Li, F. Baccelli, H. Dhillon, and J. Andrews, "Fitting determinantal point processes to macro base station deployments," in IEEE Global Commun. Conf. (GLOBECOM), pp. 3641-3646, Dec. 2014.

[17] N. Deng, W. Zhou, and M. Haenggi, "The Ginibre point process as a model for wireless networks with repulsion," IEEE Trans. Wireless Commun., vol. 14, pp. 107-121, Jan. 2015.

[18] N. Miyoshi and T. Shirai, "A cellular network model with Ginibre configured base stations," Advances in Applied Probability, vol. 46, pp. 832-845, Sept. 2014.

[19] I. Nakata and N. Miyoshi, "Spatial stochastic models for analysis of heterogeneous cellular networks with repulsively deployed base stations," Performance Evaluation, vol. 78, pp. 7-17, Aug. 2014.

[20] G. L. Torrisi and E. Leonardi, "Large deviations of the interference in the Ginibre network model," Stochastic Systems, vol. 4, no. 1, pp. 173-205, 2014.

[21] Y. Zhou, Z. Zhao, Y. Louët, Q. Ying, R. Li, X. Zhou, X. Chen, and H. Zhang, "Large-scale spatial distribution identification of base stations in cellular networks," IEEE Access, vol. 3, pp. 2987-2999, 2015.

[22] M. Haenggi, "Mean interference in hard-core wireless networks," IEEE Commun. Lett., vol. 15, pp. 792-794, Aug. 2011.

[23] F. Lagum, S. Szyszkowicz, and H. Yanikomeroglu, "CoV-based metrics for quantifying the regularity of hard-core point processes for modeling base station locations," IEEE Wireless Commun. Lett., vol. 5, pp. 276-279, June 2016.

[24] R. Ganti and M. Haenggi, "Regularity in sensor networks," in IEEE Int. Zurich Seminar Commun., pp. 186-189, Feb. 2006.

[25] M. Mirahsan, R. Schoenen, and H. Yanikomeroglu, "HetHetNets: Heterogeneous traffic distribution in heterogeneous wireless cellular networks," IEEE J. Sel. Areas Commun., vol. 33, pp. 2252-2265, Oct. 2015.

[26] M. Mirahsan, R. Schoenen, and H. Yanikomeroglu, "Statistical modeling of spatial traffic distribution with adjustable heterogeneity and b s-correlation in wireless cellular networks," in IEEE Global Commun. Conf. (GLOBECOM), pp. 3647-3652, Dec. 2014. 
[27] M. Mirahsan, Z. Wang, R. Schoenen, H. Yanikomeroglu, and M. St-Hilaire, "Unified and non-parameterized statistical modeling of temporal and spatial traffic heterogeneity in wireless cellular networks," in IEEE Int. Conf. Commun. Workshops (ICC), pp. 55-60, June 2014.

[28] Y. J. Chun, M. O. Hasna, A. Ghrayeb, and M. Di Renzo, "On modeling heterogeneous wireless networks using non-Poisson point processes." 2015.

[29] J. Riihijärvi and P. Mähönen, "A spatial statistics approach to characterizing and modeling the structure of cognitive wireless networks," Ad Hoc Networks, vol. 10, pp. 858-869, July 2012.

[30] A. Busson and G. Chelius, "Point processes for interference modeling in CSMA/CA ad-hoc networks," in PE-WASUN, pp. 33-40, Oct. 2009.

[31] J. Verma, Data analysis in management with SPSS software. Springer Science \& Business Media, 2012.

[32] K. Krishnamoorthy, Handbook of statistical distributions with applications. Chapman \& Hall/CRC, 2nd ed., 2015.

[33] P. J. Clark and F. C. Evans, "Distance to nearest neighbor as a measure of spatial relationships in populations," Ecology, vol. 35, no. 4, pp. 445-453, 1954.

[34] P. M. Dixon, "Nearest neighbor methods," in Encyclopedia of Environmetrics (A. H. El-Shaarawi and W. W. Piegorsch, eds.), vol. 3, pp. 1370-1383, John Wiley \& Sons, 2002.

[35] S. Chiu, D. Stoyan, W. Kendall, and J. Mecke, Stochastic Geometry and Its Applications. Wiley Series in Probability and Statistics, Wiley, 2013.

[36] C. Barr, Applications of Voronoi Tessellations in Point Pattern Analysis. UMI dissertation services, UMI, 2008.

[37] H. ElSawy, E. Hossain, and M. Haenggi, "Stochastic geometry for modeling, analysis, and design of multi-tier and cognitive cellular wireless networks: A survey," IEEE Commun. Surveys Tuts., vol. 15, pp. 996-1019, July 2013.

[38] A. Ibrahim, T. ElBatt, and A. El-Keyi, "Coverage probability analysis for wireless networks using repulsive point processes," in IEEE Annu. Int. Symp. Pers., Indoor, Mobile Radio Commun. (PIMRC), pp. 1002-1007, Sept. 2013.

[39] T. V. Nguyen and F. Baccelli, "On the spatial modeling of wireless networks by random packing models," in IEEE INFOCOM, pp. 28-36, Mar. 2012.

[40] P. J. Diggle, Statistical Analysis of Spatial and Spatio- Temporal Point Patterns. Chapman \& Hall/CRC, 3rd ed., 2013.

[41] F. Lagum, S. S. Szyszkowicz, and H. Yanikomeroglu, "Quantifying the regularity of perturbed triangular lattices using CoV-based metrics for modeling the locations of base stations in HetNets," in IEEE Veh. Technol. Conf. (VTC), Sept. 2016. 
[42] S. A. Banani, R. S. Adve, and A. W. Eckford, "A perturbed hexagonal lattice to model basestation locations in real-world cellular networks," in IEEE Globecom Workshops (GC Wkshps), pp. 1-6, Dec. 2015.

[43] G. R. Grimmett and I. Manolescu, "Inhomogeneous bond percolation on square, triangular and hexagonal lattices," Annals of Probability, vol. 41, pp. 2990-3025, July 2013.

[44] F. Lagum, S. Szyszkowicz, and H. Yanikomeroglu, "A metric for measuring the spatial relationship among the base stations and its applications," under review in IEEE Trans. Wireless Commun., submitted Sept. 2018.

[45] M. Haenggi, "The mean interference-to-signal ratio and its key role in cellular and amorphous networks," IEEE Wireless Commun. Lett., vol. 3, pp. 597-600, Sept. 2014.

[46] R. K. Ganti and M. Haenggi, "SIR asymptotics in general cellular network models," in IEEE Int. Symp. Inf. Theory (ISIT), pp. 1009-1013, June 2015.

[47] H. Wei, N. Deng, W. Zhou, and M. Haenggi, "Approximate SIR analysis in general heterogeneous cellular networks," IEEE Trans. Commun., vol. 64, pp. 12591273, Mar. 2016.

[48] R. K. Ganti and M. Haenggi, "Asymptotics and approximation of the SIR distribution in general cellular networks," IEEE Trans. Wireless Commun., vol. 15, pp. 2130-2143, Mar. 2016.

[49] A. Achtzehn, J. Riihijärvi, and P. Mähönen, "Large-scale cellular network modeling from population data: An empirical analysis," IEEE Commun. Lett., vol. 20, pp. 2292-2295, Nov. 2016.

[50] L. M. Correia, D. Zeller, O. Blume, D. Ferling, Y. Jading, I. Gódor, G. Auer, and L. Van Der Perre, "Challenges and enabling technologies for energy aware mobile radio networks," IEEE Commun. Mag., vol. 48, Nov. 2010.

[51] C. Han, T. Harrold, S. Armour, I. Krikidis, S. Videv, P. M. Grant, H. Haas, J. S. Thompson, I. Ku, C.-X. Wang, et al., "Green radio: radio techniques to enable energy-efficient wireless networks," IEEE Commun. Mag., vol. 49, May 2011.

[52] Z. Hasan, H. Boostanimehr, and V. K. Bhargava, "Green cellular networks: A survey, some research issues and challenges," IEEE Commun. Surveys Tuts., vol. 13, no. 4, pp. 524-540, 2011.

[53] M. H. Alsharif, R. Nordin, and M. Ismail, "Survey of green radio communications networks: techniques and recent advances," Journal of computer networks and communications, vol. 2013, Nov. 2013.

[54] T. Beitelmal, S. S. Szyszkowicz, D. G. G., and H. Yanikomeroglu, "Sector and site switch-off regular patterns for energy saving in cellular networks," IEEE Trans. Wireless Commun., vol. 17, pp. 2932-2945, May 2018. 
[55] D. González, J. Hämäläinen, H. Yanikomeroglu, M. García-Lozano, and G. Senarath, "A novel multiobjective cell switch-off framework for cellular networks," IEEE Access, vol. 4, pp. 7883-7898, 2016.

[56] Ł. Budzisz et al., "Dynamic resource provisioning for energy efficiency in wireless access networks: A survey and an outlook," IEEE Commun. Surveys Tuts., vol. 16, pp. 2259-2285, 4th quarter 2014.

[57] S. Cho and W. Choi, "Energy-efficient repulsive cell activation for heterogeneous cellular networks," IEEE J. Sel. Areas Commun., vol. 31, pp. 870-882, May 2013.

[58] S. S. Kumar and A. Kumar, "Energy efficient rate coverage with base station switching and load sharing in cellular networks," in IEEE Int. Conf. Commun. Syst. and Netw. (COMSNETS), pp. 1-6, Jan. 2016.

[59] F. Lagum, Q.-N. Le-The, T. Beitelmal, S. S. Szyszkowicz, and H. Yanikomeroglu, "Cell switch-off for networks deployed with variable spatial regularity," IEEE Wireless Commun. Lett., vol. 6, pp. $234-237$, Apr. 2017.

[60] A. Kumar and C. Rosenberg, "Energy and throughput trade-offs in cellular networks using base station switching," IEEE Trans. Mobile Comput., vol. 15, pp. 364-376, Feb. 2016.

[61] E. Erkut, Y. Ülküsal, and O. Yenicerioğlu, "A comparison of p-dispersion heuristics," Computers $\& 5$ Operations Research, vol. 21, pp. 1103-1113, Dec. 1994.

[62] M. J. Kuby, "Programming models for facility dispersion: The p-dispersion and maxisum dispersion problems," Geographical Analysis, vol. 19, no. 4, pp. 315329, 1987.

[63] "Guidelines for evaluation of radio interface technologies for IMT-Advanced," Tech. Rep. ITU-R M.2135-1, ITU, Dec. 2009.

[64] Q. N. Le-The, T. Beitelmal, F. Lagum, S. S. Szyszkowicz, and H. Yanikomeroglu, "Cell switch-off algorithms for spatially irregular base station deployments," IEEE Wireless Commun. Lett., vol. 6, pp. 354-357, June 2017.

[65] J. Andrews et al., "What will 5G be?," IEEE J. Sel. Areas Commun., vol. 32, pp. 1065-1082, June 2014.

[66] X. Ge, S. Tu, G. Mao, C.-X. Wang, and T. Han, "5G ultra-dense cellular networks," IEEE Wireless Commun., vol. 23, pp. 72-79, Feb. 2016.

[67] N. Bhushan, J. Li, D. Malladi, R. Gilmore, D. Brenner, A. Damnjanovic, R. Sukhavasi, C. Patel, and S. Geirhofer, "Network densification: the dominant theme for wireless evolution into 5G," IEEE Commun. Mag., vol. 52, pp. 82-89, Feb. 2014.

[68] I. Bor-Yaliniz and H. Yanikomeroglu, "The new frontier in RAN heterogeneity: Multi-tier drone-cells," IEEE Commun. Mag., vol. 54, pp. 48-55, Nov. 2016. 
[69] R. I. Bor-Yaliniz, A. El-Keyi, and H. Yanikomeroglu, "Efficient 3-D placement of an aerial base station in next generation cellular networks," in IEEE Int. Conf. on Commun. (ICC), May 2016.

[70] A. Al-Hourani, S. Kandeepan, and A. Jamalipour, "Modeling air-to-ground path loss for low altitude platforms in urban environments," in IEEE Global Commun. Conf. (GLOBECOM), pp. 2898-2904, Dec. 2014.

[71] A. Al-Hourani, S. Kandeepan, and S. Lardner, "Optimal LAP altitude for maximum coverage," IEEE Wireless Commun. Lett., vol. 3, pp. 569-572, Dec. 2014.

[72] M. Mozaffari et al., "Efficient deployment of multiple unmanned aerial vehicles for optimal wireless coverage," IEEE Commun. Lett., vol. 20, pp. 1647-1650, Aug. 2016.

[73] M. Alzenad, A. El-Keyi, F. Lagum, and H. Yanikomeroglu, "3D placement of an unmanned aerial vehicle base station (UAV-BS) for energy-efficient maximal coverage," IEEE Wireless Commun. Lett., vol. 6, pp. 434-437, May 2017.

[74] J. Lyu, Y. Zeng, R. Zhang, and T. J. Lim, "Placement optimization of UAVmounted mobile base stations," IEEE Commun. Lett., vol. 21, pp. 604-607, Mar. 2017.

[75] E. Kalantari, H. Yanikomeroglu, and A. Yongacoglu, "On the number and 3D placement of drone base stations in wireless cellular networks," in IEEE Veh. Technol. Conf. (VTC), IEEE, Sept. 2016.

[76] R. Ghanavi, E. Kalantari, M. Sabbaghian, H. Yanikomeroglu, and A. Yongacoglu, "Efficient 3D aerial base station placement considering users mobility by reinforcement learning," in IEEE Wireless Commun. Netw. Conf. (WCNC), April 2018.

[77] X. Li, D. Guo, H. Yin, and G. Wei, "Drone-assisted public safety wireless broadband network," in IEEE Wireless Commun. and Netw. Conf. Workshops (WCNCW), pp. 323-328, Mar. 2015.

[78] Y. Zeng, R. Zhang, and T. J. Lim, "Throughput maximization for UAV-enabled mobile relaying systems," IEEE Trans. Commun., vol. 64, pp. 4983-4996, Dec. 2016.

[79] V. Sharma, M. Bennis, and R. Kumar, "UAV-assisted heterogeneous networks for capacity enhancement," IEEE Commun. Lett., vol. 20, pp. 1207-1210, June 2016.

[80] F. Lagum, I. Bor-Yaliniz, and H. Yanikomeroglu, "Strategic densification with UAV-BSs in cellular networks," IEEE Wireless Commun. Lett., vol. 7, pp. 384387, June 2018.

[81] D. Pisinger, "Upper bounds and exact algorithms for p-dispersion problems," Computers $\&$ Operations Research, vol. 33, pp. 1380-1398, May 2006. 